\title{
Au(I) Catalyzed Cyclization of Epoxyalkynes to Allylic Alcohol Containing Spiroketals and Application to the Total Synthesis of (-)-Alotaketal A
}

\author{
Jaeyeon Lee, Jiheon Kim and Hee-Yoon Lee* \\ Department of Chemistry, Korea Advanced Institute of Science and Technology (KAIST), \\ Daejeon, 305-701, Korea \\ *Email : leehy@kaist.ac.kr
}

\section{Table of Contents}

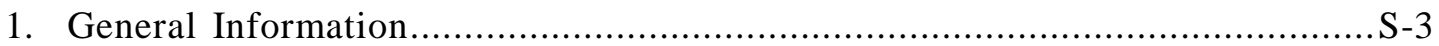

2. Experimental Procedures and Data of 28a-d............................................. -4

3. Experimental Procedures and Data of 4a-d.............................................. -5

4. Experimental Procedures and Data of 5a-d and 6a-d.................................... -7

5. Experimental Procedures and Data of 30.................................................

6. Experimental Procedures and Data of 31................................................

7. Experimental Procedures and Data of 32 .............................................

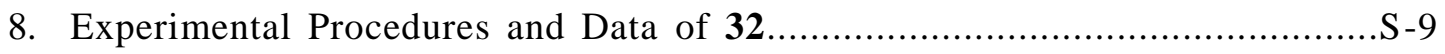

9. Experimental Procedures and Data of 33 .................................................. -10

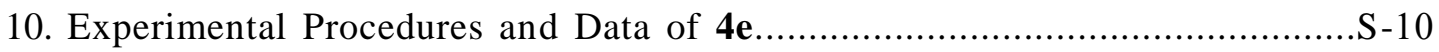

11. Experimental Procedures and Data of 6e.....................................................

12. Experimental Procedures and Data of 36...............................................

13. Experimental Procedures and Data of 37........................................... 12

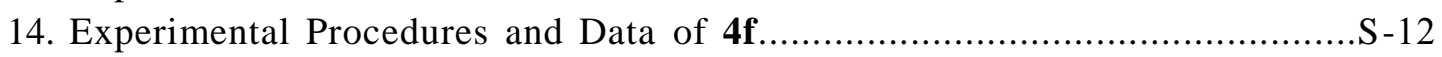

15. Experimental Procedures and Data of 5f................................................ 13

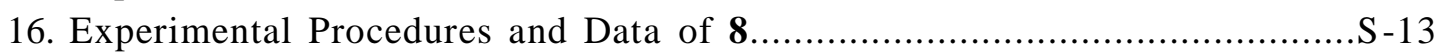

17. Experimental Procedures and Data of 40 ..................................................... 14

18. Experimental Procedures and Data of 9................................................ -14

19. Experimental Procedures and Data of 10 ................................................... 15

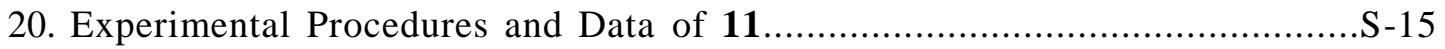

21. Experimental Procedures and Data of 12 ................................................. 16

22. Experimental Procedures and Data of 13............................................. 
23. Experimental Procedures and Data of $\mathbf{1 4}$

24. Experimental Procedures and Data of 17................................................. -18

25. Determination of Enantiomeric Ratio of $\mathbf{1 7}$ by Mosher Ester Method..........................S-19

26. Experimental Procedures and Data of 18...................................................... -20

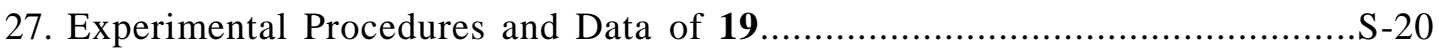

28. Experimental Procedures and Data of 1............................................... 21

29. NMR Data Comparison of Alotaketal A.....................................................S-23

30. ${ }^{1} \mathrm{H}$ NMR Spectrum Comparison of Alotaketal A....................................................... 24

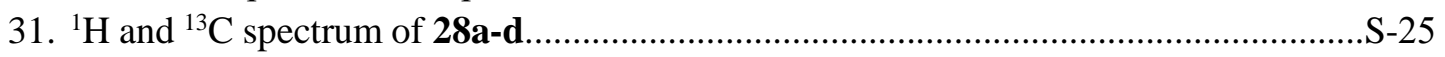

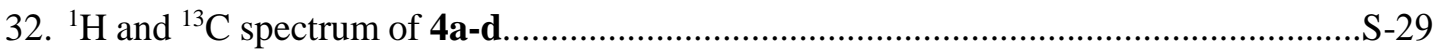

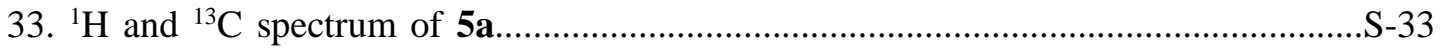

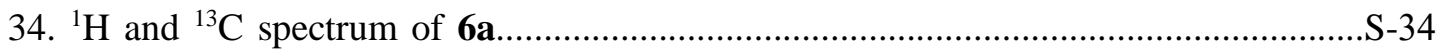

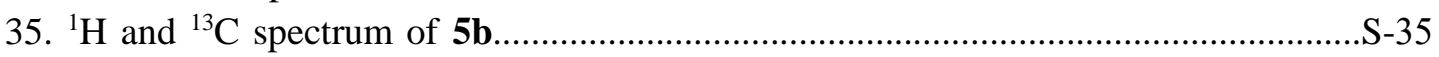

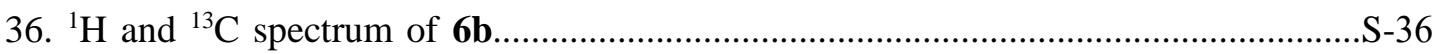

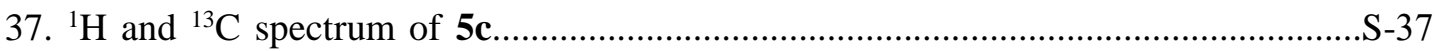

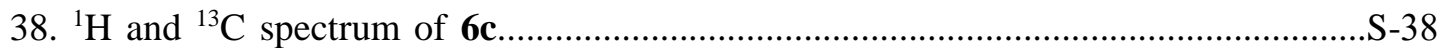

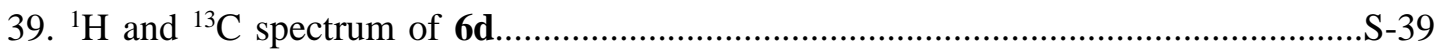

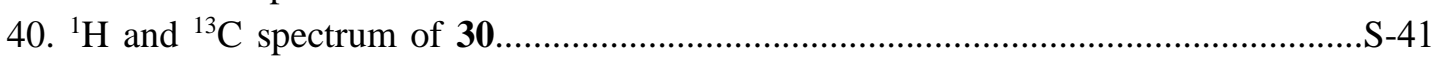

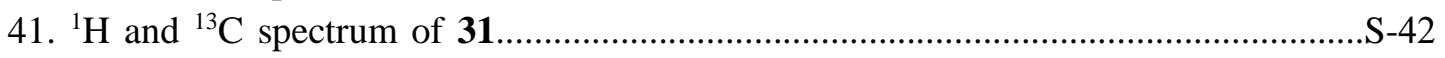

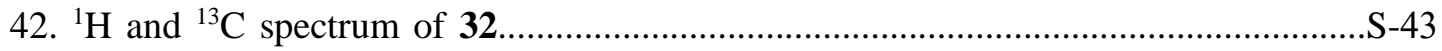

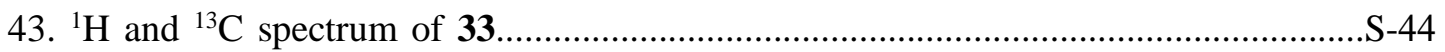

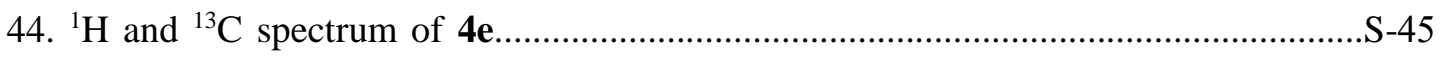

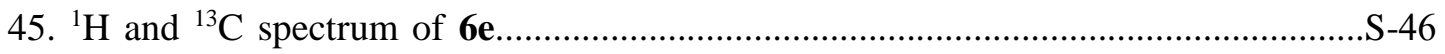

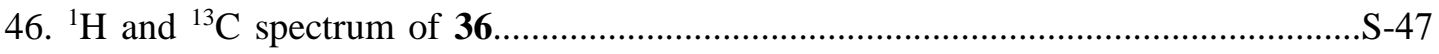

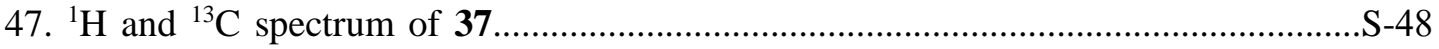

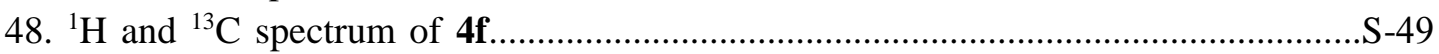

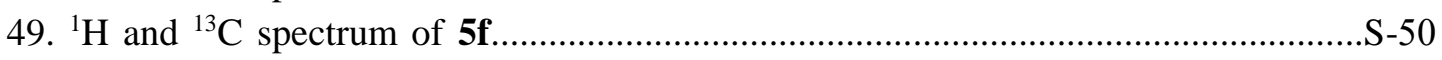

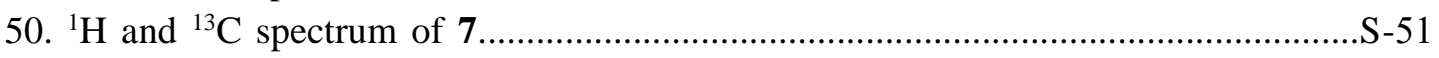

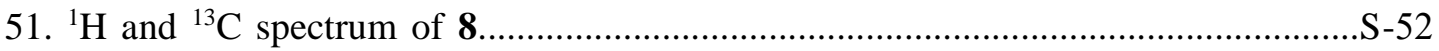

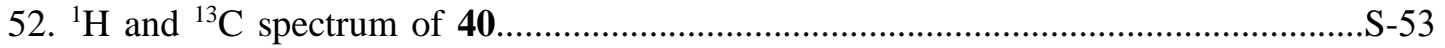

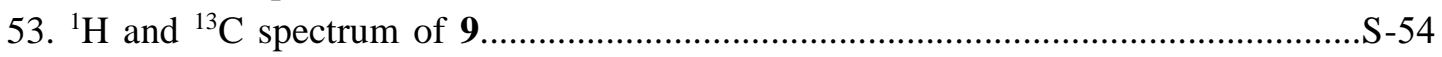

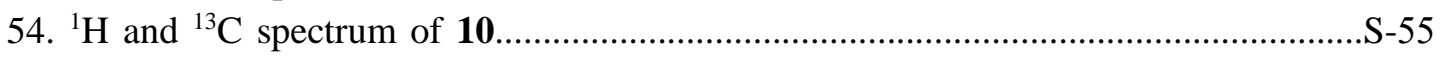

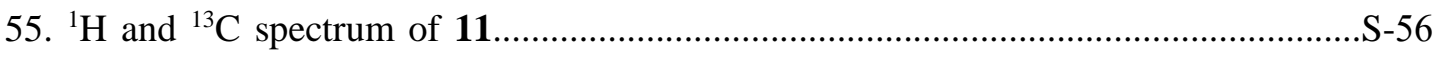

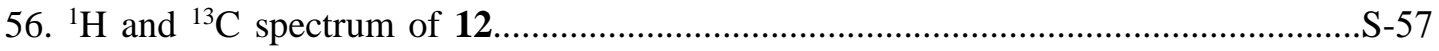

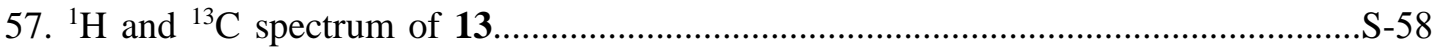

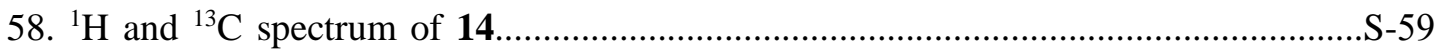

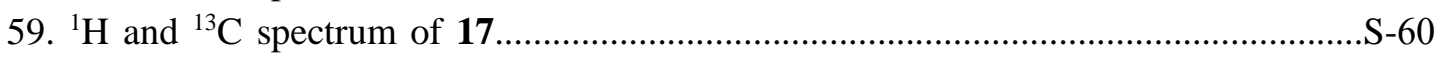

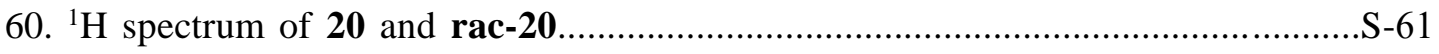

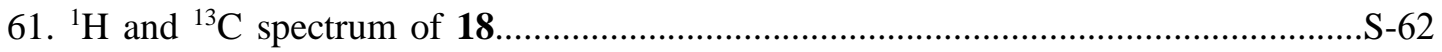

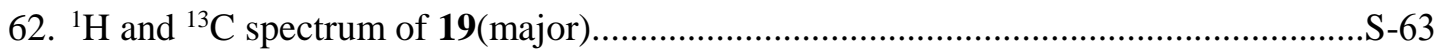

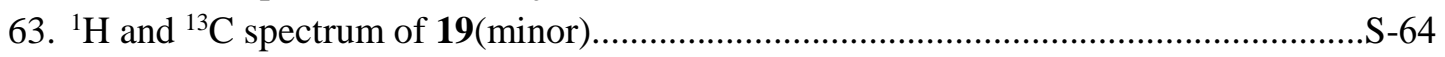

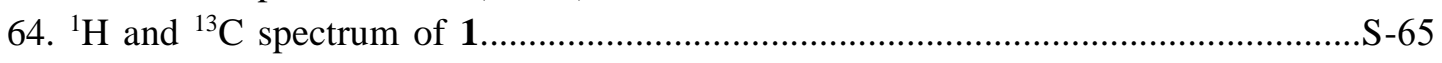

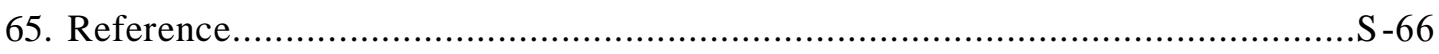




\section{General Information}

\subsection{General procedures}

All oxygen or moisture sensitive reactions were carried out in oven dried glassware under a positive pressure of argon. Sensitive liquids and solutions were transferred by syringe or cannula and were introduced through rubber septa through which a high flow of inert gas was maintained. Unless otherwise stated, reactions were carried out at room temperature. Concentration of solutions was accomplished using a Büchi rotary evaporator. This was generally followed by removal of residual solvents on a vacuum line held at 0.1-1 torr.

\subsection{Reagents and solvents}

Unless otherwise noted, all reagents and solvents were used without additional purification. Exceptions include: chromatography grade hexane and ethyl acetate were technical grade and distilled before use; $\mathrm{Et}_{2} \mathrm{O}$ and THF were distilled from sodium benzophenone ketyl under nitrogen; triethylamine were distilled from sodium; dichloromethane was distilled from $\mathrm{P}_{2} \mathrm{O}_{5}$. Concentration of alkyllithium solutions was determined by titration against diphenylacetic acid.

\subsection{Chromatography}

Analytical thin layer chromatography (TLC) was performed on Merck precoated Analytical thin layer chromatography (TLC) was performed on Merck precoated silica gel 60 F254 plates. Visualization on TLC was achieved by use of UV light $(254 \mathrm{~nm})$, exposure to iodine vapor, or treatment with acidic anisaldehyde or ceric ammonium molydate stain followed by heating. Flash column chromatography was carried out using Merck 60, 230-400 mesh ASTM.

\subsection{Physical and spectroscopic measurements}

${ }^{1} \mathrm{H}$ NMR was recorded on Bruker Avance 400 (400 MHz), Bruker Ascend 400 (400 MHz), or Agilent Technologies DD2 $(600 \mathrm{MHz})$. Chemical shifts were reported in $\delta$ units, parts per million (ppm) relative to the singlet as $7.24 \mathrm{ppm}$ for chloroform- $d$ or $7.15 \mathrm{ppm}$ for benzene- $d$. The following abbreviations were used to describe peak patterns when appropriate: $\mathrm{b}=$ broad, $\mathrm{s}=$ singlet, $\mathrm{d}=$ doublet, $\mathrm{t}=$ triplet, $\mathrm{q}$ $=$ quadraplet, $\mathrm{m}=$ multiplet. Coupling constant, $J$, was reported in Hertz unit $(\mathrm{Hz}) \cdot{ }^{13} \mathrm{C}$ NMR was recorded on Bruker Avance 400 (100 MHz), Bruker Ascend 400 (100 MHz), or Agilent Technologies $\mathrm{DD} 2(150 \mathrm{MHz})$ and was fully decoupled by broad-band decoupling. Chemical shifts were reported in ppm with the centerline of the triplet for chloroform- $d$ set at $77.00 \mathrm{ppm}$ or benzene- $d$ set at $128.00 \mathrm{ppm}$. High resolution mass spectra were obtained from KAIST Research Analysis Center by using ESI method. The optical rotations were measured using JASCO P-2000 polarimeter. 


\section{Experimental Procedures and Data}

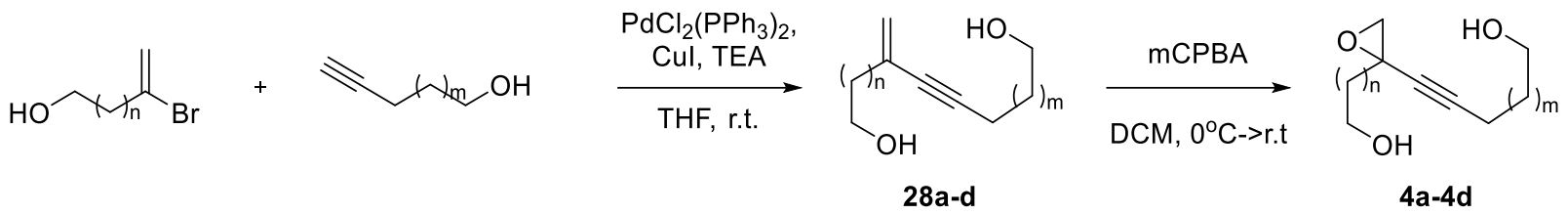

General procedure of Compound 28a-d

To a stirred solution of bromo alcohol compound $(1.00 \mathrm{mmol})$ in THF $(5 \mathrm{~mL})$, TEA $(15.00 \mathrm{mmol})$, $\mathrm{PdCl}_{2}\left(\mathrm{PPh}_{3}\right)(0.02 \mathrm{mmol}), \mathrm{CuI}(0.05 \mathrm{mmol})$ and alkynyl alochol $(1.5 \mathrm{mmol})$ were added to the reaction mixture. After $5 \mathrm{~h}$., the reaction mixture was quenched with brine, saturated $\mathrm{NH}_{4} \mathrm{Cl}$ solution and the organic layer was separated and extracted with $\mathrm{Et}_{2} \mathrm{O}$. The combined organic layer was dried over $\mathrm{MgSO}_{4}$, filtered, and concentrated under reduced pressure. The residue was purified by column chromatography on silica gel (EtOAc : Hexane $=1: 1)$ to give 28a-d.<smiles>C=C(C#CCCCO)CCO</smiles>

$48 \%$ yield, $319.7 \mathrm{mg}$ obtained, pale yellowish oil

${ }^{1}$ H NMR (500 MHz, Benzene- $\left.d_{6}\right) \delta 5.37(\mathrm{~s}, 1 \mathrm{H}), 5.05(\mathrm{~s}, 1 \mathrm{H}), 3.67(\mathrm{t}, J=6.3 \mathrm{~Hz}, 2 \mathrm{H}), 3.26(\mathrm{~d}, J=5.8$ $\mathrm{Hz}, 2 \mathrm{H}), 2.24(\mathrm{t}, J=6.3 \mathrm{~Hz}, 2 \mathrm{H}), 2.08(\mathrm{~d}, J=5.6 \mathrm{~Hz}, 2 \mathrm{H}), 1.43-1.37(\mathrm{~m}, 4 \mathrm{H})$.

${ }^{13}$ C NMR (126 MHz, Benzene- $\left.d_{6}\right) \delta$ 129.4, 121.9, 90.9, 81.4, 62.0, 61.1, 41.3, 32.1, 25.3, 19.2.

High Resolution MS (ESI-TOF) m/z: $[\mathrm{M}+\mathrm{Na}]^{+} \mathrm{Calcd}$ for $\mathrm{C}_{10} \mathrm{H}_{16} \mathrm{O}_{2} \mathrm{Na}$ 191.1048; Found: 191.1035<smiles>C=C(CO)CCCO</smiles>

$75 \%$ yield, 29.5mg obtained, pale yellow oil

${ }^{1}$ H NMR $\left(400 \mathrm{MHz}\right.$, Benzene- $\left.d_{6}\right) \delta 5.36(\mathrm{~s}, 1 \mathrm{H}), 5.06(\mathrm{~s}, 1 \mathrm{H}), 3.66(\mathrm{t}, J=6.7 \mathrm{~Hz}, 2 \mathrm{H}), 3.39(\mathrm{t}, J=6.0$ $\mathrm{Hz}, 2 \mathrm{H}), 2.24$ (t, $J=6.3 \mathrm{~Hz}, 2 \mathrm{H}), 2.20$ (t, $J=7.0 \mathrm{~Hz}, 2 \mathrm{H}), 1.47$ (p, $J=6.5 \mathrm{~Hz}, 2 \mathrm{H})$.

${ }^{13}$ C NMR (101 MHz, Benzene- $\left.d_{6}\right) \delta 129.3,122.0,90.5,81.4,61.2,61.1,41.2,31.7,16.0$.

High Resolution MS (ESI-TOF) m/z: $[\mathrm{M}+\mathrm{Na}]^{+} \mathrm{Calcd}$ for $\mathrm{C}_{9} \mathrm{H}_{14} \mathrm{O}_{2} \mathrm{Na}$ 177.0886; Found: 177.0888<smiles>C=C(CO)CCCCCCCCCCCCO</smiles>

49\% yield, $22.4 \mathrm{mg}$ obtained, pale yellow oil 
${ }^{1}$ H NMR (400 MHz, Benzene- $\left.d_{6}\right) \delta 5.39(\mathrm{~s}, 1 \mathrm{H}), 5.09(\mathrm{~s}, 1 \mathrm{H}), 3.75(\mathrm{t}, J=6.4 \mathrm{~Hz}, 2 \mathrm{H}), 3.45-3.34(\mathrm{~m}$, $2 \mathrm{H}), 3.27(\mathrm{t}, J=5.6 \mathrm{~Hz}, 1 \mathrm{H}), 2.31(\mathrm{t}, J=6.4 \mathrm{~Hz}, 2 \mathrm{H}), 2.18-2.05(\mathrm{~m}, 2 \mathrm{H}), 1.94(\mathrm{t}, J=6.0 \mathrm{~Hz}, 1 \mathrm{H})$, $1.33(\mathrm{~d}, J=3.4 \mathrm{~Hz}, 6 \mathrm{H})$.

${ }^{13}$ C NMR (101 MHz, Benzene- $\left.d_{6}\right) \delta 129.4,121.8,91.0,81.4,62.3,61.1,41.3,32.4,28.6,25.2,19.4$.

High Resolution MS (ESI-TOF) m/z: [M+Na] ${ }^{+} \mathrm{Calcd}$ for $\mathrm{C}_{11} \mathrm{H}_{18} \mathrm{O}_{2} \mathrm{Na}$ 205.1199; Found: 205.1191<smiles>C=C(C#CCCCO)CO</smiles>

28d

$62 \%$ yield, $115.8 \mathrm{mg}$, pale yellow oil

${ }^{1}$ H NMR (400 MHz, Benzene- $\left.d_{6}\right) \delta 5.46-5.42(\mathrm{~m}, 1 \mathrm{H}), 5.37(\mathrm{q}, J=1.8 \mathrm{~Hz}, 1 \mathrm{H}), 3.97(\mathrm{~s}, 2 \mathrm{H}), 3.27$ (t, $J=5.9 \mathrm{~Hz}, 2 \mathrm{H}), 2.15-2.05(\mathrm{~m}, 2 \mathrm{H}), 1.39$ (p, $J=3.2 \mathrm{~Hz}, 4 \mathrm{H})$.

${ }^{13}$ C NMR $\left(101 \mathrm{MHz}\right.$, Benzene- $\left.d_{6}\right) \delta 132.6,118.4,91.9,79.5,65.5,61.9,32.0,25.2,19.3$.

High Resolution MS (ESI-TOF) m/z: $[\mathrm{M}+\mathrm{Na}]^{+}$Calcd for $\mathrm{C}_{9} \mathrm{H}_{14} \mathrm{O}_{2} \mathrm{Na}$ 177.0886; Found: 177.0889

General procedure of Compound 4a-d

To a stirred solution of 28a-d (1eq.) in $\mathrm{DCM}(0.1 \mathrm{M})$ at $0^{\circ} \mathrm{C}, \mathrm{mCPBA}$ (purity $<77 \%, 1.2$ eq.) was added to the reaction mixture. After $4 \mathrm{~h}$, the reaction mixture was quenched with brine, saturated $\mathrm{NaHCO}_{3}$ solution and the organic layer was separated and extracted with Ethyl acetate. The combined organic layer was dried over $\mathrm{MgSO}_{4}$, filtered, and concentrated under reduced pressure. The residue was purified by column chromatography on silica gel (EtOAc : Hexane $=2: 1)$ to give 4a-d.<smiles>OCCCC1CO1</smiles>

$34 \%$ yield, 114.1 mg obtained, pale yellowish oil

${ }^{1}$ H NMR (400 MHz, Benzene- $\left.d_{6}\right) \delta 3.67(\mathrm{t}, J=6.1 \mathrm{~Hz}, 2 \mathrm{H}), 3.22(\mathrm{t}, J=6.0 \mathrm{~Hz}, 2 \mathrm{H}), 2.74(\mathrm{~d}, J=5.4$ $\mathrm{Hz}, 1 \mathrm{H}), 2.36(\mathrm{~d}, J=5.6 \mathrm{~Hz}, 1 \mathrm{H}), 1.90(\mathrm{t}, J=6.7 \mathrm{~Hz}, 2 \mathrm{H}), 1.80-1.65(\mathrm{~m}, 2 \mathrm{H}), 1.32(\mathrm{dt}, J=6.0,2.7$ $\mathrm{Hz}, 4 \mathrm{H})$.

${ }^{13}$ C NMR (126 MHz, Benzene- $\left.d_{6}\right) \delta 84.2,79.6,61.9,59.5,54.0,49.3,39.6,31.9,25.0,18.5$.

High Resolution MS (ESI-TOF) m/z: [M+Na] ${ }^{+}$Calcd for $\mathrm{C}_{10} \mathrm{H}_{16} \mathrm{O}_{3} \mathrm{Na} 207.0997$; Found: 207.0981<smiles>OCCCC1(CO)CO1</smiles>

$42 \%$ yield, $13.2 \mathrm{mg}$ obtained, colorless oil

${ }^{1} \mathbf{H}$ NMR $\left(400 \mathrm{MHz}\right.$, Benzene- $\left.d_{6}\right) \delta 3.67(\mathrm{t}, J=6.3 \mathrm{~Hz}, 2 \mathrm{H}), 3.32(\mathrm{t}, J=6.1 \mathrm{~Hz}, 2 \mathrm{H}), 2.72(\mathrm{~d}, J=5.6$ $\mathrm{Hz}, 1 \mathrm{H}), 2.36(\mathrm{~d}, J=5.6 \mathrm{~Hz}, 1 \mathrm{H}), 2.03(\mathrm{t}, J=7.0 \mathrm{~Hz}, 2 \mathrm{H}), 1.72(\mathrm{qt}, J=14.2,6.1 \mathrm{~Hz}, 2 \mathrm{H}), 1.39(\mathrm{q}, J=$ $6.5,6.0 \mathrm{~Hz}, 2 \mathrm{H})$. 
${ }^{13}$ C NMR (101 MHz, Benzene- $\left.d_{6}\right) \delta 83.8,79.6,65.9,61.0,59.5,53.9,39.5,31.3,15.3$.

High Resolution MS (ESI-TOF) m/z: [M+Na] ${ }^{+}$Calcd for $\mathrm{C}_{9} \mathrm{H}_{14} \mathrm{O}_{3} \mathrm{Na}$ 193.0835; Found: 193.0837<smiles>OCCCCCCCO</smiles>

$82 \%$ yield, $16.1 \mathrm{mg}$ obtained, colorless oil

${ }^{1}$ H NMR (400 MHz, Benzene- $\left.d_{6}\right) \delta 3.78-3.67(\mathrm{~m}, 2 \mathrm{H}), 3.37-3.19(\mathrm{~m}, 2 \mathrm{H}), 2.76(\mathrm{dd}, J=5.7,0.7 \mathrm{~Hz}$, $1 \mathrm{H}), 2.37(\mathrm{~d}, J=5.7 \mathrm{~Hz}, 1 \mathrm{H}), 1.99-1.87(\mathrm{~m}, 2 \mathrm{H}), 1.83-1.68(\mathrm{~m}, 2 \mathrm{H}), 1.25(\mathrm{~d}, J=2.8 \mathrm{~Hz}, 6 \mathrm{H})$.

${ }^{13}$ C NMR (101 MHz, Benzene- $\left.d_{6}\right) \delta 84.2,79.6,62.3,59.5,54.0,49.3,39.6,32.3,28.3,25.1,18.7$.

High Resolution MS (ESI-TOF) m/z: [M+Na] ${ }^{+}$Calcd for $\mathrm{C}_{11} \mathrm{H}_{18} \mathrm{O}_{3} \mathrm{Na} 221.1148$; Found: 221.1152<smiles>OCCCC#CC1(CO)CO1</smiles>

4d

$75 \%$ yield, $15.2 \mathrm{mg}$ obtained, colorless oil

${ }^{1}$ H NMR (400 MHz, Benzene- $\left.d_{6}\right) \delta 3.69(\mathrm{~d}, J=12.4 \mathrm{~Hz}, 1 \mathrm{H}), 3.56(\mathrm{~d}, J=12.4 \mathrm{~Hz}, 1 \mathrm{H}), 3.27(\mathrm{t}, J=5.9$ $\mathrm{Hz}, 2 \mathrm{H}), 2.70(\mathrm{~d}, J=5.8 \mathrm{~Hz}, 1 \mathrm{H}), 2.65(\mathrm{~d}, J=5.8 \mathrm{~Hz}, 1 \mathrm{H}), 1.99-1.87(\mathrm{~m}, 2 \mathrm{H}), 1.34(\mathrm{dtd}, J=6.7,3.1$, $1.5 \mathrm{~Hz}, 4 \mathrm{H})$.

${ }^{13}$ C NMR (101 MHz, Benzene- $\left.d_{6}\right) \delta 85.5,77.6,63.9,61.9,51.4,51.0,31.8,24.9,18.6$.

High Resolution MS (ESI-TOF) m/z: $[\mathrm{M}+\mathrm{Na}]^{+}$Calcd for $\mathrm{C}_{9} \mathrm{H}_{14} \mathrm{O}_{3} \mathrm{Na}$ 193.0835; Found: 193.0838

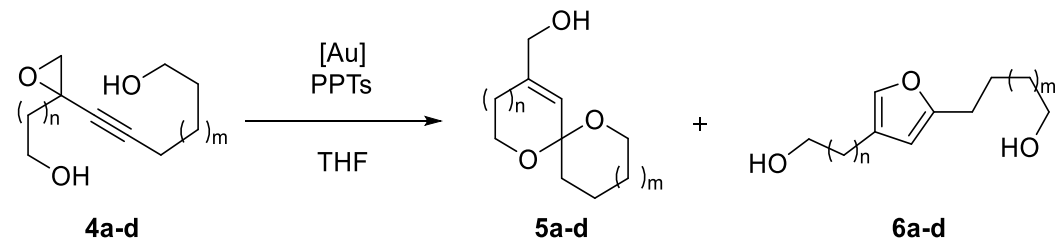

\section{General procedure}

To a stirred solution of gold catalyst (and $\mathrm{AgSbF}_{6}$, if included) and PPT in THF were added diol epoxy alkyne compound, 4a-d. The resulting mixture was stirred for $5 \mathrm{~min}$. The reaction mixture was diluted with $\mathrm{Et}_{2} \mathrm{O}$ and saturated $\mathrm{NaHCO}_{3}$ solution. The aqueous layer was extracted with $\mathrm{Et}_{2} \mathrm{O}$ and then the combined organic layer was dried over $\mathrm{MgSO}_{4}$ and filtered. The filtrate was concentrated under reduced pressure. The residue was purified by flash column chromatography on silica gel (EtOAc : Hexane $=$ $1: 1$ to $2: 1$ ) to afford 5a-d and 6a-d. 


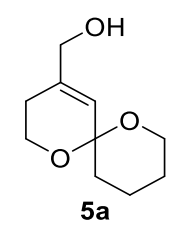

$80.1 \%$ yield, $27.1 \mathrm{mg}$ obtained, colorless oil

${ }^{1}$ H NMR (400 MHz, Benzene- $\left.d_{6}\right) \delta 5.65(\mathrm{dq}, J=2.5,1.4 \mathrm{~Hz}, 1 \mathrm{H}), 3.95-3.85(\mathrm{~m}, 2 \mathrm{H}), 3.69(\mathrm{~s}, 2 \mathrm{H})$, $3.66(\mathrm{dd}, J=11.0,6.3 \mathrm{~Hz}, 1 \mathrm{H}), 3.58(\mathrm{ddd}, J=10.8,6.1,1.4 \mathrm{~Hz}, 1 \mathrm{H}), 2.06-1.92$ (m, 2H), $1.79-1.71$ (m, 1H), $1.61-1.39(\mathrm{~m}, 4 \mathrm{H}), 1.31-1.20(\mathrm{~m}, 1 \mathrm{H})$.

${ }^{13}$ C NMR (101 MHz, Benzene- $\left.d_{6}\right) \delta 139.2,125.0,93.5,65.5,60.8,57.8,35.6,25.5,25.4,19.1$.

High Resolution MS (ESI-TOF) m/z: [M+Na] ${ }^{+} \mathrm{Calcd}$ for $\mathrm{C}_{10} \mathrm{H}_{16} \mathrm{O}_{3} \mathrm{Na}$ 207.0997; Found: 207.0985<smiles>OCCCCc1cc(CCCO)co1</smiles>

$6.2 \%$ yield, $1.80 \mathrm{mg}$ obtained, colorless oil

${ }^{1}$ H NMR (400 MHz, Benzene- $\left.d_{6}\right) \delta 6.98(\mathrm{~s}, 1 \mathrm{H}), 5.76(\mathrm{~s}, 1 \mathrm{H}), 3.45(\mathrm{t}, J=6.5 \mathrm{~Hz}, 2 \mathrm{H}), 3.24(\mathrm{t}, J=6.4$ $\mathrm{Hz}, 2 \mathrm{H}), 2.43(\mathrm{t}, J=7.5 \mathrm{~Hz}, 2 \mathrm{H}), 2.36(\mathrm{td}, J=6.5,0.7 \mathrm{~Hz}, 2 \mathrm{H}), 1.60-1.48(\mathrm{~m}, 2 \mathrm{H}), 1.30$ (dq, $J=10.1$, $6.4 \mathrm{~Hz}, 2 \mathrm{H})$.

${ }^{13}$ C NMR (101 MHz, Benzene- $\left.d_{6}\right) \delta 156.7,138.1,122.5,106.9,62.6,62.2,32.4,28.9,28.1,24.6$.

High Resolution MS (ESI): Calculated for $\mathrm{C}_{10} \mathrm{H}_{16} \mathrm{O}_{3}[\mathrm{M}+\mathrm{Na}]^{+}:$207.0997, Found: 207.0989

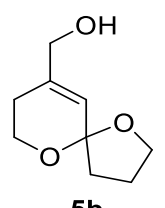

$5 b$

$64 \%$ yield, $8.4 \mathrm{mg}$ obtained, colorless oil

${ }^{1} \mathbf{H}$ NMR $\left(400 \mathrm{MHz}\right.$, Benzene- $\left.d_{6}\right) \delta 5.59(\mathrm{q}, J=1.2 \mathrm{~Hz}, 1 \mathrm{H}), 4.03$ (ddd, $J=12.1,11.1,3.6$ $\mathrm{Hz}, 1 \mathrm{H}), 3.94(\mathrm{td}, J=8.0,5.1 \mathrm{~Hz}, 1 \mathrm{H}), 3.85-3.75(\mathrm{~m}, 1 \mathrm{H}), 3.68(\mathrm{ddd}, J=11.2,6.1,1.1$ $\mathrm{Hz}, 1 \mathrm{H}), 3.64(\mathrm{~s}, 2 \mathrm{H}), 2.05-1.89(\mathrm{~m}, 3 \mathrm{H}), 1.72-1.49(\mathrm{~m}, 2 \mathrm{H}), 1.42(\mathrm{dd}, J=17.1,3.6 \mathrm{H}$ $\mathrm{z}, 1 \mathrm{H})$.

${ }^{13}$ C NMR (101 MHz, Benzene- $\left.d_{6}\right) \delta 140.3,122.9,67.3,65.5,59.0,38.1,30.4,25.2,24.9$.

High Resolution MS (ESI-TOF) m/z: [M+Na $]^{+}$Calcd for $\mathrm{C}_{9} \mathrm{H}_{14} \mathrm{O}_{3} \mathrm{Na}$ 193.0835; Found: 193.0837<smiles>OCCc1coc(CCO)c1</smiles>

6b

$10 \%$ yield, $1.3 \mathrm{mg}$ obtained, colorless oil

${ }^{1}$ H NMR $\left(400 \mathrm{MHz}\right.$, Benzene- $\left.d_{6}\right) \delta 6.98(\mathrm{~s}, 1 \mathrm{H}), 5.77 \mathrm{zj}(\mathrm{s}, 1 \mathrm{H}), 3.48(\mathrm{t}, J=6.5 \mathrm{~Hz}, 2 \mathrm{H}), 3.31(\mathrm{t}, J=6.2$ $\mathrm{Hz}, 2 \mathrm{H}), 2.55(\mathrm{t}, J=7.5 \mathrm{~Hz}, 2 \mathrm{H}), 2.37(\mathrm{td}, J=6.5,1.0 \mathrm{~Hz}, 2 \mathrm{H}), 1.66(\mathrm{tt}, J=7.5,6.2 \mathrm{~Hz}, 2 \mathrm{H})$.

${ }^{13}$ C NMR (101 MHz, Benzene- $\left.d_{6}\right) \delta 138.2,132.4,122.6,107.1,62.6,61.7,30.2,28.9,24.7$

High Resolution MS (ESI-TOF) m/z: $[\mathrm{M}+\mathrm{Na}]^{+} \mathrm{Calcd}$ for $\mathrm{C}_{9} \mathrm{H}_{14} \mathrm{O}_{3} \mathrm{Na}$ 193.0835; Found: 193.0840 
<smiles>CCOC12CCCCCC1=C(CO)O2</smiles>

$46 \%$ yield, $7.45 \mathrm{mg}$ obtained, colorless oil

${ }^{1}$ H NMR $\left(400 \mathrm{MHz}\right.$, Benzene- $\left.d_{6}\right) \delta 5.86-5.80(\mathrm{~m}, 1 \mathrm{H}), 4.04(\mathrm{ddd}, J=12.2,10.9,3.6 \mathrm{~Hz}, 1 \mathrm{H}), 3.87$ (ddd, $J=12.7,11.7,1.2 \mathrm{~Hz}, 1 \mathrm{H}), 3.71-3.62(\mathrm{~m}, 3 \mathrm{H}), 3.59(\mathrm{dtd}, J=12.7,3.3,1.7 \mathrm{~Hz}, 1 \mathrm{H}), 2.01-1.94$ (m, 2H), $1.70-1.59(\mathrm{~m}, 2 \mathrm{H}), 1.51-1.42(\mathrm{~m}, 4 \mathrm{H}), 1.36-1.28(\mathrm{~m}, 1 \mathrm{H}), 1.20-1.08(\mathrm{~m}, 1 \mathrm{H})$.

${ }^{13}$ C NMR (101 MHz, Benzene- $\left.d_{6}\right) \delta 136.6,124.3,98.2,65.6,62.3,58.2,40.0,31.3,30.2,25.5,22.6$.

High Resolution MS (ESI-TOF) m/z: [M+Na] ${ }^{+}$Calcd for $\mathrm{C}_{11} \mathrm{H}_{18} \mathrm{O}_{3} \mathrm{Na} 221.1148$; Found: 221.1149<smiles>OCCCCCc1cc(CCCO)co1</smiles>

$8 \%$ yield, $1.24 \mathrm{mg}$ obtained, colorless oil

${ }^{1}$ H NMR $\left(400 \mathrm{MHz}\right.$, Benzene- $\left.d_{6}\right) \delta 7.00(\mathrm{~s}, 1 \mathrm{H}), 5.79(\mathrm{~s}, 1 \mathrm{H}), 3.49(\mathrm{t}, J=6.5 \mathrm{~Hz}, 2 \mathrm{H}), 3.28(\mathrm{t}, J=6.4$ $\mathrm{Hz}, 2 \mathrm{H}), 2.46(\mathrm{t}, J=7.5 \mathrm{~Hz}, 2 \mathrm{H}), 2.39(\mathrm{t}, J=6.5 \mathrm{~Hz}, 2 \mathrm{H}), 1.51$ (p, $J=7.6 \mathrm{~Hz}, 2 \mathrm{H}), 1.29$ (dt, $J=13.2$, $6.6 \mathrm{~Hz}, 2 \mathrm{H}), 1.20(\mathrm{dt}, J=13.7,6.7 \mathrm{~Hz}, 2 \mathrm{H})$.

${ }^{13}$ C NMR (101 MHz, Benzene- $\left.d_{6}\right) \delta 156.5,137.8,122.2,106.6,62.3,62.1,32.4,30.1,28.6,28.0,27.8$, 25.3.

High Resolution MS (ESI-TOF) m/z: [M+Na] ${ }^{+} \mathrm{Calcd}$ for $\mathrm{C}_{11} \mathrm{H}_{18} \mathrm{O}_{3} \mathrm{Na} 221.1148$; Found: 221.1149<smiles>OCCCCc1cc(CO)co1</smiles>

$88 \%$ yield, $7.8 \mathrm{mg}$ obtained, colorless oil

${ }^{1} \mathrm{H}$ NMR $\left(599 \mathrm{MHz}\right.$, Benzene- $\left.d_{6}\right) \delta 7.02(\mathrm{~s}, 1 \mathrm{H}), 5.89(\mathrm{~s}, 1 \mathrm{H}), 4.19(\mathrm{~s}, 2 \mathrm{H}), 3.25(\mathrm{t}, J=6.4 \mathrm{~Hz}, 2 \mathrm{H})$, $2.42(\mathrm{t}, J=7.5 \mathrm{~Hz}, 2 \mathrm{H}), 1.58-1.47(\mathrm{~m}, 2 \mathrm{H}), 1.37-1.22(\mathrm{~m}, 2 \mathrm{H})$.

${ }^{13}$ C NMR $\left(151 \mathrm{MHz}\right.$, Benzene- $\left.\boldsymbol{d}_{6}\right) \delta$ 137.97, 128.29, 105.61, 62.22, 56.82, 32.35, 28.04, 24.54. (one of peaks was buried by $\mathrm{C}_{6} \mathrm{D}_{6}$ solvent peaks) ${ }^{13} \mathbf{C}$ NMR $(151 \mathrm{MHz}$, Chloroform- $d) \delta 157.0,138.1$, 125.7, 105.2, 62.5, 56.8, 32.1, 27.7, 24.2.

High Resolution MS (ESI-TOF) m/z: [M+Na $]^{+}$Calcd for $\mathrm{C}_{9} \mathrm{H}_{14} \mathrm{O}_{3} \mathrm{Na}$ 193.0835; Found: 193.0838 

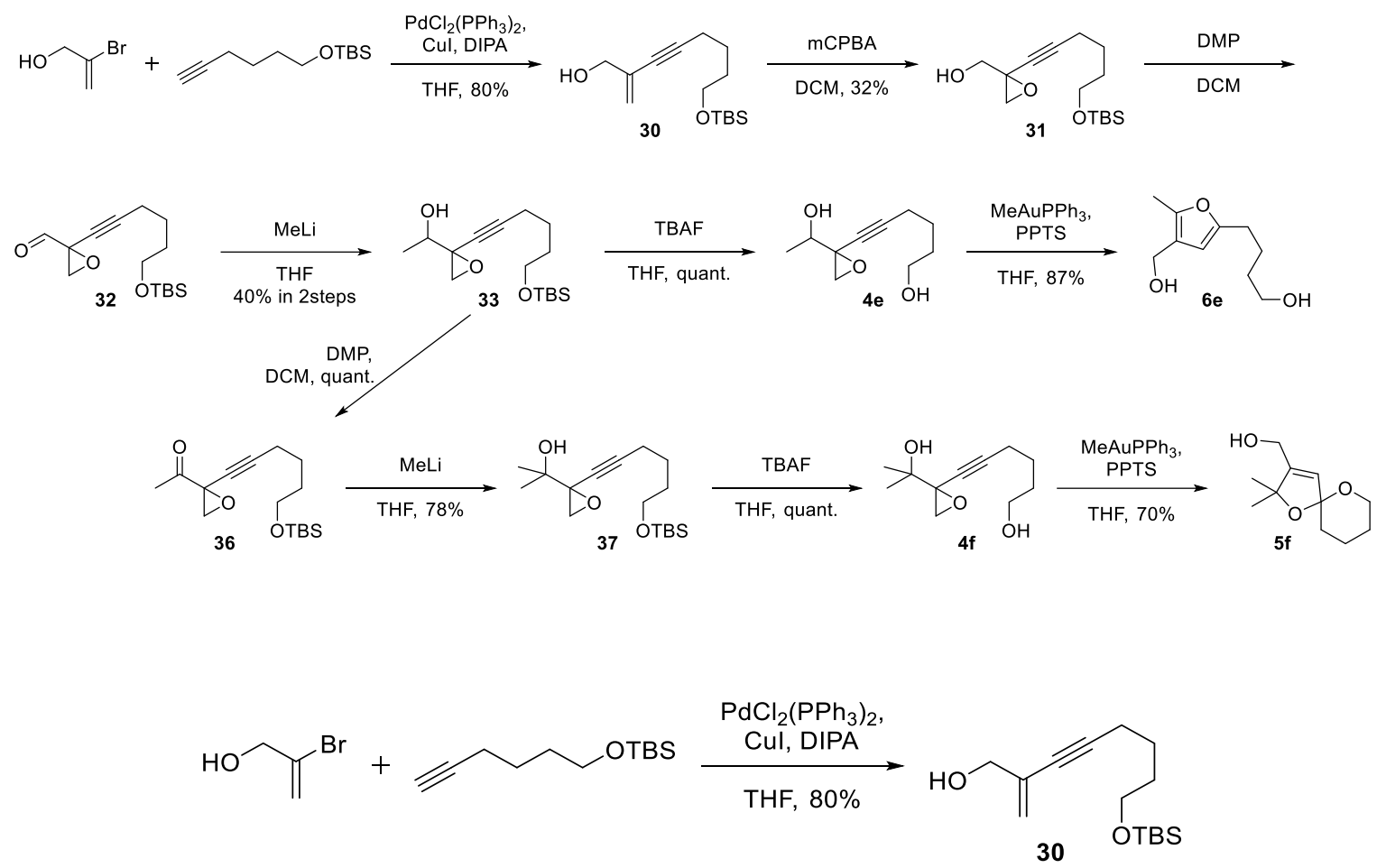

30 : Followed by general procedure of Compound 26a-d, $80 \%$ yield, $1.2 \mathrm{~g}$ obtained, pale yellowish oil ${ }^{1}$ H NMR $\left(599 \mathrm{MHz}\right.$, Benzene- $\left.d_{6}\right) \delta 5.42(\mathrm{~d}, J=1.6 \mathrm{~Hz}, 1 \mathrm{H}), 5.39$ (q, $\left.J=1.8 \mathrm{~Hz}, 1 \mathrm{H}\right), 3.97(\mathrm{~s}, 2 \mathrm{H}), 3.46$ (t, $J=6.0 \mathrm{~Hz}, 2 \mathrm{H}), 2.15(\mathrm{t}, J=6.7 \mathrm{~Hz}, 1 \mathrm{H}), 1.60-1.37(\mathrm{~m}, 4 \mathrm{H}), 0.95(\mathrm{~s}, 9 \mathrm{H}), 0.03(\mathrm{~s}, 6 \mathrm{H})$.

${ }^{13}$ C NMR $\left(151 \mathrm{MHz}\right.$, Benzene- $\left.d_{6}\right) \delta 85.4,77.6,63.5,62.6,51.5,50.9,32.1,26.1,25.2,18.6,-5.2$.

High Resolution MS (ESI-TOF) m/z: [M+Na] ${ }^{+}$Calcd for $\mathrm{C}_{15} \mathrm{H}_{28} \mathrm{O}_{2} \mathrm{SiNa} 291.1756$; Found: 291.1759<smiles>C=C(C#CCCCOCCCCCCCO)CO</smiles><smiles>CCCCCC1(CO)CO1</smiles>

31 : Followed by general procedure of Compound 4a-d, 32\% yield, 410mg obtained, colorless oil

${ }^{1} \mathbf{H}$ NMR $\left(599 \mathrm{MHz}\right.$, Benzene- $\left.d_{6}\right) \delta 3.73(\mathrm{~d}, J=12.5 \mathrm{~Hz}, 1 \mathrm{H}), 3.61(\mathrm{~d}, J=12.5 \mathrm{~Hz}, 1 \mathrm{H}), 3.43(\mathrm{t}, J=5.9$ $\mathrm{Hz}, 2 \mathrm{H}), 2.74(\mathrm{~d}, J=5.9 \mathrm{~Hz}, 1 \mathrm{H}), 2.70(\mathrm{~d}, J=5.8 \mathrm{~Hz}, 1 \mathrm{H}), 1.98(\mathrm{t}, J=6.8 \mathrm{~Hz}, 2 \mathrm{H}), 1.45(\mathrm{ddtd}, J=22.0$, 8.7, 6.6, 6.1, 2.8 Hz, 4H), 0.94 (s, 9H), $0.02(\mathrm{~s}, 6 \mathrm{H})$.

${ }^{13}$ C NMR (151 MHz, Benzene- $\left.d_{6}\right) \delta 85.4,77.6,63.5,62.6,51.5,50.9,32.1,26.1,25.2,18.6,-5.2$.

High Resolution MS (ESI-TOF) m/z: [M+Na] $]^{+}$Calcd for $\mathrm{C}_{15} \mathrm{H}_{28} \mathrm{O}_{3} \mathrm{SiNa}$ 307.1705; Found: 307.1699<smiles>OCC1(C#CCCCCO[Sb])CO1</smiles>

31<smiles>O=CC1(C#CCCCC[AsH3+])CO1</smiles>

32

To a solution of the alcohol compound, $31(80.40 \mathrm{mg}, 0.283 \mathrm{mmol})$ in DCM (4 mL) was added Dess- 
Martin periodinane (DMP; $179.85 \mathrm{mg}, 0.424 \mathrm{mmol}$ ) at $0^{\circ} \mathrm{C}$ and then allowed warm to room temperature. After stirring for $3 \mathrm{~h}$, the reaction mixture was quenched with saturated $\mathrm{Na}_{2} \mathrm{~S}_{2} \mathrm{O}_{3}$, and the organic layer was separated and extracted with DCM. The combined organic layer was dried over $\mathrm{MgSO}_{4}$, filtered, and concentrated under reduced pressure. The residue was purified by column chromatography on silica gel $($ EtOAc : Hexane $=1: 10)$ to give $32(75.94 \mathrm{mg}, 95 \%$, pale yellowish oil).

${ }^{1}$ H NMR $\left(599 \mathrm{MHz}\right.$, Benzene- $\left.d_{6}\right) \delta 8.32(\mathrm{~s}, 1 \mathrm{H}), 3.42(\mathrm{t}, J=6.0 \mathrm{~Hz}, 2 \mathrm{H}), 2.65(\mathrm{~d}, J=5.7 \mathrm{~Hz}, 1 \mathrm{H}), 2.21$ $(\mathrm{d}, J=5.7 \mathrm{~Hz}, 1 \mathrm{H}), 1.98(\mathrm{t}, J=6.9 \mathrm{~Hz}, 2 \mathrm{H}), 1.53-1.47(\mathrm{~m}, 2 \mathrm{H}), 1.47-1.41(\mathrm{~m}, 2 \mathrm{H}), 0.96(\mathrm{~s}, 9 \mathrm{H})$, $0.03(\mathrm{~s}, 6 \mathrm{H})$.

${ }^{13}$ C NMR (151 MHz, Benzene- $\left.d_{6}\right) \delta 192.7,89.1,72.7,62.6,52.8,51.9,32.0,26.1,25.0,18.7,18.4,-$ 5.3.

High Resolution MS (ESI-TOF) m/z: [M+Na] ${ }^{+}$Calcd for $\mathrm{C}_{15} \mathrm{H}_{26} \mathrm{O}_{3} \mathrm{SiNa}$ 305.1549; Found: 305.1544<smiles>O=CC1(C#CCCCC[SeH])CO1</smiles>

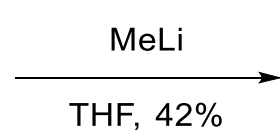

THF, $42 \%$<smiles>CC(O)C1(C#CCCCC[Sb])CO1</smiles>

To a solution of the alcohol compound, 32(58.58mg, $0.207 \mathrm{mmol})$ in THF ( $2 \mathrm{~mL}$ ) was added MeLi ( 0.2 $\mathrm{mL}$ of $1.6 \mathrm{M}$ solution in $\mathrm{Hx}, 0.32 \mathrm{mmol})$ at $-78^{\circ} \mathrm{C}$ and then allowed warm to room temperature. After stirring for $4 \mathrm{~h}$, the reaction mixture was quenched with saturated $\mathrm{NH}_{4} \mathrm{Cl}$, and the organic layer was separated and extracted with $\mathrm{Et}_{2} \mathrm{O}$. The combined organic layer was dried over $\mathrm{MgSO}_{4}$, filtered, and concentrated under reduced pressure. The residue was purified by column chromatography on silica gel (EtOAc : Hexane $=1: 20$ ) to give $\mathbf{3 3}$ (26mg, 42\%, yellowish oil).

${ }^{1}$ H NMR $\left(599 \mathrm{MHz}\right.$, Benzene- $\left.d_{6}\right) \delta 3.80(\mathrm{q}, J=6.3 \mathrm{~Hz}, 1 \mathrm{H}), 3.43(\mathrm{t}, J=5.9 \mathrm{~Hz}, 2 \mathrm{H}), 2.70(\mathrm{~d}, J=5.8$ $\mathrm{Hz}, 1 \mathrm{H}), 2.67(\mathrm{~d}, J=5.8 \mathrm{~Hz}, 1 \mathrm{H}), 1.97(\mathrm{t}, J=6.8 \mathrm{~Hz}, 2 \mathrm{H}), 1.52-1.39(\mathrm{~m}, 4 \mathrm{H}), 1.33(\mathrm{~d}, J=6.3 \mathrm{~Hz}, 31 \mathrm{H})$, 0.96 (s, 9H), 0.03 (s, 6H).

${ }^{13}$ C NMR $\left(151 \mathrm{MHz}\right.$, Benzene- $\left.d_{6}\right) \delta 85.7,77.7,67.8,62.6,54.6,50.3,32.1,26.1,25.2,19.1,18.6,18.4$, -5.2 .

High Resolution MS (ESI-TOF) m/z: [M+Na] ${ }^{+}$Calcd for $\mathrm{C}_{16} \mathrm{H}_{30} \mathrm{O}_{3} \mathrm{SiNa}$ 321.1862; Found: 321.1863
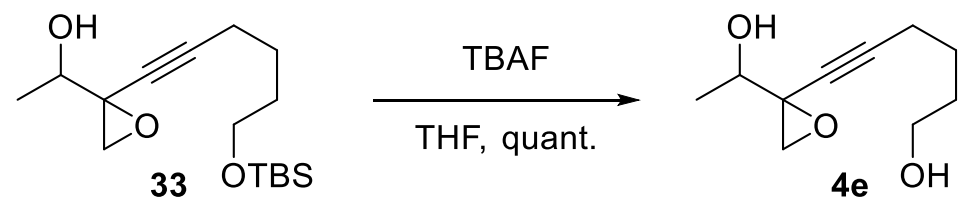

To a solution of $\mathbf{3 3}(8.20 \mathrm{mg}, 0.027 \mathrm{mmol})$ in THF ( $1 \mathrm{~mL}$ ) was added tetra-n-butylammonium fluoride (TBAF; $5.20 \mathrm{~mL}$ of $1.0 \mathrm{M}$ in THF, $5.19 \mathrm{mmol}$ ) and stirred for $3 \mathrm{~h}$. The reaction mixture was quenched with saturated $\mathrm{NH}_{4} \mathrm{Cl}$, and the organic layer was separated and extracted with $\mathrm{Et}_{2} \mathrm{O}$. The combined organic layer was dried over $\mathrm{MgSO}_{4}$, filtered, and concentrated under reduced pressure. The residue was purified by column chromatography on silica gel (EtOAc : Hexane =1:1) to give $\mathbf{3 4}(4.97 \mathrm{mg}$, quant.). 
${ }^{1}$ H NMR $\left(599 \mathrm{MHz}\right.$, Benzene- $\left.d_{6}\right) \delta 3.51(\mathrm{q}, J=6.4 \mathrm{~Hz}, 1 \mathrm{H}), 3.25(\mathrm{t}, J=5.8 \mathrm{~Hz}, 2 \mathrm{H}), 2.70(\mathrm{~d}, J=5.8$ $\mathrm{Hz}, 1 \mathrm{H}), 2.56(\mathrm{~d}, J=5.8 \mathrm{~Hz}, 1 \mathrm{H}), 1.92(\mathrm{t}, J=6.5 \mathrm{~Hz}, 2 \mathrm{H}), 1.38-1.31(\mathrm{~m}, 4 \mathrm{H}), 1.28(\mathrm{~d}, J=6.4 \mathrm{~Hz}$, $3 \mathrm{H})$.

${ }^{13}$ C NMR (101 MHz, Chloroform- $d$ ) $\delta 165.96,143.89,132.76,130.41,130.36,129.62,128.16,123.43$, $121.42,82.85,78.02,70.19,68.39,42.33,31.49,18.85$.

High Resolution MS (ESI): Calculated for $\mathrm{C}_{10} \mathrm{H}_{16} \mathrm{O}_{3}[\mathrm{M}+\mathrm{Na}]^{+}:$207.0997, Found: 207.0996
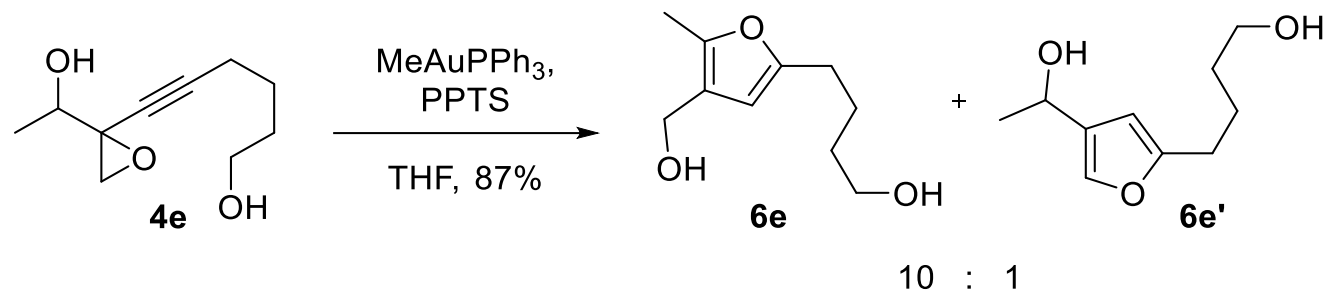

To a solution of $4 \mathbf{e}(7.60 \mathrm{mg}, 0.041 \mathrm{mmol})$ in THF $(1 \mathrm{~mL})$ was added pyridinium $p$-toluenesulfonate (PPTs; $10.50 \mathrm{mg}, 0.042 \mathrm{mmol})$ and $\mathrm{MeAuPPh}_{3}(19.60 \mathrm{mg}, 0.041 \mathrm{mmol})$. The resulting mixture was stirred for $5 \mathrm{~min}$. The reaction mixture was diluted with $\mathrm{Et}_{2} \mathrm{O}$ and saturated $\mathrm{NaHCO}_{3}$ solution. The aqueous layer was extracted with $\mathrm{Et}_{2} \mathrm{O}$ and then the combined organic layer was dried over $\mathrm{MgSO}_{4}$ and filtered. The filtrate was concentrated under reduced pressure. The residue was purified by flash column chromatography on silica gel (EtOAc : Hexane $=1: 1$ ) to afford the inseparable mixture of $\mathbf{6 e}$ and $\mathbf{6 e}^{\prime}(6.57 \mathrm{mg}, 87 \%$, the ratio was measured by $1 \mathrm{H}$ NMR in the ratio of $10: 1)$.

${ }^{1}$ H NMR $\left(599 \mathrm{MHz}\right.$, Benzene- $\left.d_{6}\right) \delta 5.90(\mathrm{~s}, 1 \mathrm{H}), 4.20(\mathrm{~s}, 2 \mathrm{H}), 3.28(\mathrm{t}, J=6.4 \mathrm{~Hz}, 2 \mathrm{H}), 2.45(\mathrm{t}, J=7.6$ $\mathrm{Hz}, 2 \mathrm{H}), 2.00$ (s, 3H), 1.58 (dt, $J=15.2,7.6 \mathrm{~Hz}, 2 \mathrm{H}), 1.35$ (dt, $J=13.6,6.5 \mathrm{~Hz}, 2 \mathrm{H})$.

${ }^{13}$ C NMR (151 MHz, Benzene- $\left.d_{6}\right) \delta 154.03,146.83,120.27,106.65,62.28,56.69,32.42,28.01,24.72$, 11.41 .

High Resolution MS (ESI): Calculated for $\mathrm{C}_{10} \mathrm{H}_{16} \mathrm{O}_{3}[\mathrm{M}+\mathrm{Na}]^{+}:$207.0997, Found: 207.0993
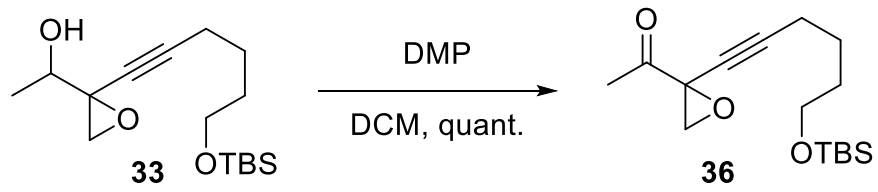

To a solution of the alcohol compound, 33(13.60 mg, $0.046 \mathrm{mmol})$ in DCM (4 mL) was added DessMartin periodinane (DMP; $29.30 \mathrm{mg}, 0.069 \mathrm{mmol}$ ) at $0{ }^{\circ} \mathrm{C}$ and then allowed warm to room temperature. After stirring for $4 \mathrm{~h}$, the reaction mixture was quenched with saturated $\mathrm{Na}_{2} \mathrm{~S}_{2} \mathrm{O}_{3}$, and the organic layer was separated and extracted with DCM. The combined organic layer was dried over $\mathrm{MgSO}_{4}$, filtered, and concentrated under reduced pressure. The residue was purified by column chromatography on silica gel (EtOAc : Hexane $=1: 10$ ) to give 36 (13.64 mg, quant., pale yellowish oil).

${ }^{1}$ H NMR $\left(599 \mathrm{MHz}\right.$, Benzene- $\left.d_{6}\right) \delta 3.44(\mathrm{t}, J=5.9 \mathrm{~Hz}, 2 \mathrm{H}), 2.66(\mathrm{~d}, J=6.3 \mathrm{~Hz}, 1 \mathrm{H}), 2.42(\mathrm{dd}, J=6.4$, $0.9 \mathrm{~Hz}, 1 \mathrm{H}), 2.00(\mathrm{t}, J=6.8 \mathrm{~Hz}, 2 \mathrm{H}), 1.71(\mathrm{~s}, 3 \mathrm{H}), 1.51(\mathrm{dq}, J=10.3,5.9 \mathrm{~Hz}, 2 \mathrm{H}), 1.48-1.42(\mathrm{~m}, 2 \mathrm{H})$, $0.96(\mathrm{~s}, 9 \mathrm{H}), 0.03(\mathrm{~s}, 6 \mathrm{H})$. 
${ }^{13}$ C NMR $\left(151 \mathrm{MHz}\right.$, Benzene- $\left.d_{6}\right) \delta$ 200.0, 86.9, 75.4, 62.6, 53.6, 32.1, 26.1, 25.1, 24.1, 18.7, 18.4, 5.2 .

High Resolution MS (ESI-TOF) m/z: [M+Na] ${ }^{+}$Calcd for $\mathrm{C}_{16} \mathrm{H}_{28} \mathrm{O}_{3} \mathrm{SiNa} 319.1705$; Found: 319.1709<smiles>CC(=O)C1(C#CCCCC[Sb])CO1</smiles>

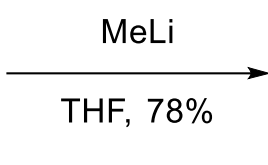<smiles>CC(O)(O)C1(C#CCCCC[SeH])CO1</smiles>

37

To a solution of the alcohol compound, $\mathbf{3 6}(8.55 \mathrm{mg}, 0.029 \mathrm{mmol})$ in THF $(1 \mathrm{~mL})$ was added MeLi $(0.03$ $\mathrm{mL}$ of $1.6 \mathrm{M}$ solution in $\mathrm{Hx}, 0.048 \mathrm{mmol})$ at $-78^{\circ} \mathrm{C}$ and then allowed warm to room temperature. After stirring for $2 \mathrm{~h}$, the reaction mixture was quenched with saturated $\mathrm{NH}_{4} \mathrm{Cl}$, and the organic layer was separated and extracted with $\mathrm{Et}_{2} \mathrm{O}$. The combined organic layer was dried over $\mathrm{MgSO}_{4}$, filtered, and concentrated under reduced pressure. The residue was purified by column chromatography on silica gel (EtOAc : Hexane $=1: 10)$ to give $\mathbf{3 7}(7.07 \mathrm{mg}, \mathbf{7 8 \%}$, pale yellowish oil).

${ }^{1} \mathbf{H}$ NMR $\left(599 \mathrm{MHz}\right.$, Benzene- $\left.d_{6}\right) \delta 3.43(\mathrm{t}, J=6.0 \mathrm{~Hz}, 2 \mathrm{H}), 2.91(\mathrm{~d}, J=6.1 \mathrm{~Hz}, 1 \mathrm{H}), 2.68(\mathrm{~d}, J=6.1$ $\mathrm{Hz}, 1 \mathrm{H}), 1.96(\mathrm{t}, J=6.9 \mathrm{~Hz}, 2 \mathrm{H}), 1.50-1.45(\mathrm{~m}, 2 \mathrm{H}), 1.43(\mathrm{~s}, 3 \mathrm{H}), 1.45-1.40(\mathrm{~m}, 2 \mathrm{H}), 1.39(\mathrm{~s}, 3 \mathrm{H})$, $0.96(\mathrm{~s}, 9 \mathrm{H}), 0.03(\mathrm{~s}, 6 \mathrm{H})$.

${ }^{13}$ C NMR $\left(151 \mathrm{MHz}\right.$, Benzene- $\left.d_{6}\right) \delta 84.9,78.4,69.7,62.6,57.3,51.2,32.1,26.9,26.1,25.5,25.3,18.6$, $18.4,-5.2$.

High Resolution MS (ESI-TOF) m/z: [M+Na] ${ }^{+}$Calcd for $\mathrm{C}_{17} \mathrm{H}_{32} \mathrm{O}_{3} \mathrm{SiNa}$ 335.2018; Found: 335.2007
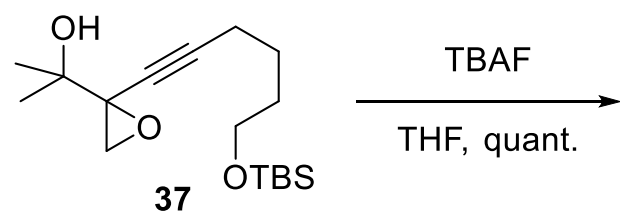<smiles>CC(C)(O)C1(C#CCCCCO)CO1</smiles>

To a solution of $37(6.10 \mathrm{mg}, 0.020 \mathrm{mmol})$ in THF (1 mL) was added tetra-n-butylammonium fluoride (TBAF; $0.06 \mathrm{~mL}$ of $1.0 \mathrm{M}$ in THF, $0.06 \mathrm{mmol}$ ) and stirred for $3 \mathrm{~h}$. The reaction mixture was quenched with saturated $\mathrm{NH}_{4} \mathrm{Cl}$, and the organic layer was separated and extracted with $\mathrm{Et}_{2} \mathrm{O}$. The combined organic layer was dried over $\mathrm{MgSO}_{4}$, filtered, and concentrated under reduced pressure. The residue was purified by column chromatography on silica gel (EtOAc : Hexane $=1: 1)$ to give $\mathbf{4 f}(3.96 \mathrm{mg}$, quant., pale yellow oil).

${ }^{1}$ H NMR $\left(599 \mathrm{MHz}\right.$, Benzene- $\left.d_{6}\right) \delta 3.26(\mathrm{t}, J=5.7 \mathrm{~Hz}, 2 \mathrm{H}), 2.92(\mathrm{~d}, J=6.0 \mathrm{~Hz}, 1 \mathrm{H}), 2.68(\mathrm{~d}, J=6.0$ $\mathrm{Hz}, 1 \mathrm{H}), 1.91(\mathrm{t}, J=6.7 \mathrm{~Hz}, 2 \mathrm{H}), 1.43(\mathrm{~s}, 3 \mathrm{H}), 1.39(\mathrm{~s}, 3 \mathrm{H}), 1.38-1.31(\mathrm{~m}, 4 \mathrm{H})$.

${ }^{13}$ C NMR (151 MHz, Benzene- $\left.d_{6}\right) \delta 84.9,78.4,69.8,61.8,57.3,51.2,32.0,26.9,25.5,25.1,18.6$.

High Resolution MS (ESI-TOF) m/z: [M+Na] ${ }^{+}$Calcd for $\mathrm{C}_{11} \mathrm{H}_{18} \mathrm{O}_{3} \mathrm{Na}$ 221.1154; Found: 221.1143 

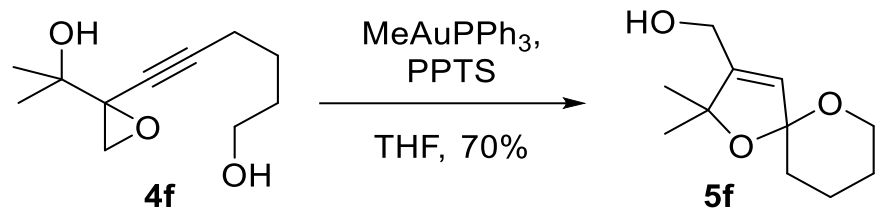

To a solution of $\mathbf{4 f}(11.40 \mathrm{mg}, 0.058 \mathrm{mmol})$ in THF $(1 \mathrm{~mL})$ was added pyridinium $p$-toluenesulfonate (PPTs; $14.8 \mathrm{mg}, 0.071 \mathrm{mmol})$ and $\mathrm{MeAuPPh}_{3}(27.30 \mathrm{mg}, 0.058 \mathrm{mmol})$. The resulting mixture was stirred for 5 min. The reaction mixture was diluted with $\mathrm{Et}_{2} \mathrm{O}$ and saturated $\mathrm{NaHCO}_{3}$ solution. The aqueous layer was extracted with $\mathrm{Et}_{2} \mathrm{O}$ and then the combined organic layer was dried over $\mathrm{MgSO}_{4}$ and filtered. The filtrate was concentrated under reduced pressure. The residue was purified by flash column chromatography on silica gel (EtOAc : Hexane $=1: 1)$ to afford $\mathbf{5 f}(8.05 \mathrm{mg}, 70 \%$, amorphous solid).

${ }^{1}$ H NMR $\left(599 \mathrm{MHz}\right.$, Benzene- $\left.d_{6}\right) \delta 5.57(\mathrm{~s}, 1 \mathrm{H}), 4.13(\mathrm{ddd}, J=11.9,11.0,2.6 \mathrm{~Hz}, 1 \mathrm{H}), 3.88-3.78(\mathrm{~m}$, $2 \mathrm{H}), 3.70(\mathrm{ddt}, J=11.1,4.4,1.9 \mathrm{~Hz}, 1 \mathrm{H}), 2.06-1.93(\mathrm{~m}, 1 \mathrm{H}), 1.79-1.61(\mathrm{~m}, 2 \mathrm{H}), 1.57-1.43(\mathrm{~m}$, $2 \mathrm{H}), 1.41(\mathrm{~s}, 3 \mathrm{H}), 1.33-1.24(\mathrm{~m}, 1 \mathrm{H}), 1.21(\mathrm{~s}, 3 \mathrm{H})$.

${ }^{13}$ C NMR (151 MHz, Benzene- $\left.d_{6}\right) \delta 151.7,124.4,107.6,86.8,62.3,58.4,36.0,28.5,27.7,25.7,20.0$.

High Resolution MS (ESI-TOF) m/z: [M+Na] ${ }^{+}$Calcd for $\mathrm{C}_{11} \mathrm{H}_{18} \mathrm{O}_{3} \mathrm{Na} 221.1154$; Found: 221.1143

\section{Compound 7}

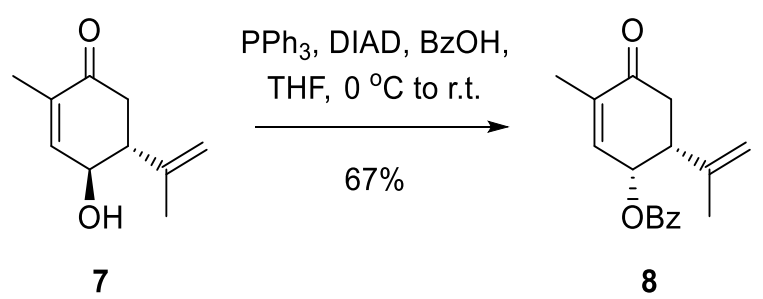

To a stirred solution of allylic alcohol starting material,7 (1.133 g, $6.82 \mathrm{mmol})$ in THF (50 $\mathrm{mL})$ were added triphenylphosphine (3.58 g, $13.63 \mathrm{mmol})$, benzoic acid (1.67 g, $13.63 \mathrm{mmol})$, and diisopropyl azodicarboxylate (DIAD; $2.68 \mathrm{~mL}, 13.63 \mathrm{mmol}$ ) at $0{ }^{\circ} \mathrm{C}$. The resulting mixture was allowed to warm to r.t. and stirred for $1 \mathrm{~h}$. The reaction mixture was diluted with $\mathrm{Et}_{2} \mathrm{O}$ and saturated $\mathrm{NaHCO}_{3}$ solution $(50 \mathrm{~mL})$. The aqueous layer was extracted with $\mathrm{Et}_{2} \mathrm{O}(50 \mathrm{~mL} \times 3)$ and then the combined organic layer was dried over $\mathrm{MgSO}_{4}$ and filtered. The filtrate was concentrated under reduced pressure. The residue was purified by flash column chromatography on silica gel (EtOAc : Hexane $=1: 15$ ) to afford 8 (1.234 g, $4.56 \mathrm{mmol}, 67 \%$, yellowish crystalline solid).

${ }^{1} \mathrm{H}$ NMR $(400 \mathrm{MHz}$, Chloroform- $d$ ) $\delta 7.96(\mathrm{~d}, J=9.6 \mathrm{~Hz}, 2 \mathrm{H}), 7.58-7.50(\mathrm{~m}, 1 \mathrm{H}), 7.41(\mathrm{t}, J=7.7 \mathrm{~Hz}$, $2 \mathrm{H}), 6.89(\mathrm{~d}, J=7.1 \mathrm{~Hz}, 1 \mathrm{H}), 5.76(\mathrm{~d}, J=2.2 \mathrm{~Hz}, 1 \mathrm{H}), 4.93(\mathrm{~s}, 1 \mathrm{H}), 4.81(\mathrm{~s}, 1 \mathrm{H}), 2.96(\mathrm{~d}, J=11.3 \mathrm{~Hz}$, $2 \mathrm{H}), 2.61(\mathrm{q}, J=11.3,10.7 \mathrm{~Hz}, 1 \mathrm{H}), 1.83(\mathrm{~s}, 3 \mathrm{H}), 1.80(\mathrm{~s}, 3 \mathrm{H})$.

${ }^{13}$ C NMR (101 MHz, Chloroform-d) $\delta$ 199.2, 165.8, 142.8, 139.0, 138.7, 133.2, 129.8 , 129.6, 128.4, $113.0,66.8,44.4,37.9,21.9,15.6$.

High Resolution MS (ESI-TOF) m/z: [M+Na] $]^{+}$Calcd for $\mathrm{C}_{17} \mathrm{H}_{18} \mathrm{O}_{3} \mathrm{Na}$ 293.1154; Found: 293.1147 $[\alpha]^{25}=-369.78\left(\mathrm{c} 1.9425, \mathrm{CH}_{2} \mathrm{Cl}_{2}\right)$ 


\section{Compound 8}

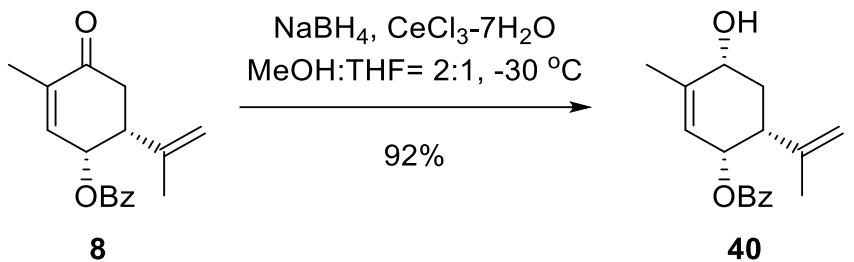

To a solution of enone, $8(1.66 \mathrm{~g}, 6.14 \mathrm{mmol})$ in $\mathrm{MeOH}(16 \mathrm{~mL})$ and THF $(8 \mathrm{~mL})$ cooled to $-30{ }^{\circ} \mathrm{C}$ was added sodium borohydride $(255.52 \mathrm{mg}, 6.75 \mathrm{mmol})$ and cerium(III) chloride heptahydrate $(2.29 \mathrm{~g}$, $6.14 \mathrm{mmol}$ ) at $-30^{\circ} \mathrm{C}$ (Eyela PSL-1810, constant temperature bath with magnetic stirrer) and the reaction mixture was stirred for $1 \mathrm{~h}$. The reaction mixture was quenched with addition of water $(2.1 \mathrm{~mL}), \mathrm{Et}_{2} \mathrm{O}$. The filtrate was concentrated under reduced pressure and the residue was purified by column chromatography on silica gel (EtOAc : Hexane $=1: 5)$ to give $\mathbf{4 0}(1.54 \mathrm{~g}, 92 \%$, pale yellow oil).

${ }^{1}$ H NMR (400 MHz, Chloroform- $d$ ) $\delta 7.99-7.93(\mathrm{~m}, 2 \mathrm{H}), 7.54-7.46(\mathrm{~m}, 1 \mathrm{H}), 7.38(\mathrm{dd}, J=8.4,7.1$ $\mathrm{Hz}, 2 \mathrm{H}), 5.79(\mathrm{dp}, J=4.7,1.6 \mathrm{~Hz}, 1 \mathrm{H}), 5.54-5.47(\mathrm{~m}, 1 \mathrm{H}), 4.84(\mathrm{q}, J=1.4 \mathrm{~Hz}, 1 \mathrm{H}), 4.81(\mathrm{q}, J=1.2$ $\mathrm{Hz}, 1 \mathrm{H}), 4.24-4.03$ (m, 1H), 2.38 (dt, $J=13.4,2.9 \mathrm{~Hz}, 1 \mathrm{H}), 2.17$ (dddd, $J=12.3,6.1,2.5,1.3 \mathrm{~Hz}, 1 \mathrm{H}$ ), $2.00(\mathrm{td}, J=12.6,10.3 \mathrm{~Hz}, 1 \mathrm{H}), 1.83(\mathrm{dt}, J=1.7,0.9,3 \mathrm{H}), 1.77-1.75(\mathrm{~m}, 3 \mathrm{H})$

${ }^{13}$ C NMR (101 MHz, Chloroform- $d$ ) $\delta$ 166.0, 144.4, 144.2, 132.6, 130.3, 129.4, 128.1, 121.2, 111.6, 70.4, 67.7, 43.2, 32.0, 21.9, 18.8 .

High Resolution MS (ESI-TOF) m/z: $[\mathrm{M}+\mathrm{Na}]^{+}$Calcd for $\mathrm{C}_{17} \mathrm{H}_{20} \mathrm{O}_{3} \mathrm{Na}$ 295.1310; Found: 295.1306 $[\alpha]^{25}=-247.81\left(\mathrm{c} 2.121, \mathrm{CH}_{2} \mathrm{Cl}_{2}\right)$

\section{Compound 9}

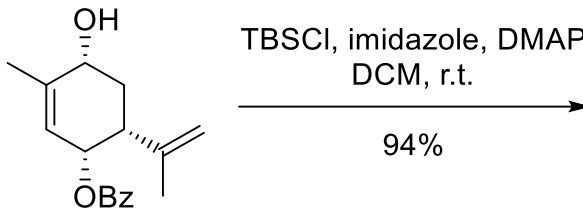

30

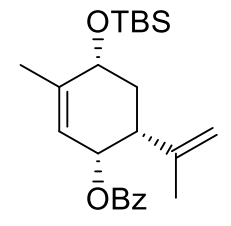

9

To a solution of allylic alcohol, $30(1.42 \mathrm{~g}, 5.21 \mathrm{mmol})$ in DCM $(20 \mathrm{~mL})$ was added tbutyldimethylsilyl chloride (TBSCl; $1.57 \mathrm{~g}, 10.43 \mathrm{mmol}$ ), 4-dimethylamine pyridine (DMAP; 319.21 $\mathrm{mg}, 2.61 \mathrm{mmol})$ and imidazole $(711.29 \mathrm{mg}, 10.44 \mathrm{mmol})$. After stirring for $3 \mathrm{~h}$, the reaction mixture was quenched with water and saturated $\mathrm{NH}_{4} \mathrm{Cl}$ solution and extracted with DCM. The combined organic layers were dried over $\mathrm{MgSO}_{4}$, filtered and concentrated under reduced pressure. The residue was purified by column chromatography on silica gel (EtOAc : Hexane $=1: 30)$ to afford $9(1.89 \mathrm{~g}, 94 \%$, pale yellow oil).

${ }^{1}$ H NMR (400 MHz, Chloroform- $d$ ) $\delta 8.02-7.93(\mathrm{~m}, 2 \mathrm{H}), 7.50(\mathrm{t}, J=6.9 \mathrm{~Hz}, 1 \mathrm{H}), 7.39(\mathrm{t}, J=7.6 \mathrm{~Hz}$, $2 \mathrm{H}), 5.74(\mathrm{~d}, J=5.2 \mathrm{~Hz}, 1 \mathrm{H}), 5.50(\mathrm{~d}, J=4.0 \mathrm{~Hz}, 1 \mathrm{H}), 4.84(\mathrm{~s}, 1 \mathrm{H}), 4.80(\mathrm{~s}, 1 \mathrm{H}), 4.23-4.12(\mathrm{~m}, 1 \mathrm{H})$, $2.35(\mathrm{~d}, J=13.7 \mathrm{~Hz}, 1 \mathrm{H}), 2.10-1.92(\mathrm{~m}, 2 \mathrm{H}), 1.76(\mathrm{~s}, 6 \mathrm{H}), 0.92(\mathrm{~s}, 9 \mathrm{H}), 0.12(\mathrm{~d}, J=4.8 \mathrm{~Hz}, 6 \mathrm{H}) ;{ }^{13} \mathrm{C}$ 
NMR (101 MHz, Chloroform- $d$ ) $\delta$ 166.1, 145.2, 144.8, 132.7, 130.6, 129.7, 128.2, 120.8, 111.6, 71.4, $67.7,43.4,32.6,25.9,22.1,19.7,18.2,-4.1,-4.9$;

High Resolution MS (ESI-TOF) m/z: [M+Na] $]^{+}$Calcd for $\mathrm{C}_{23} \mathrm{H}_{34} \mathrm{O}_{3} \mathrm{SiNa}$ 409.2175; Found: 409.2164 $[\alpha]_{D}^{25}=-226.61\left(\mathrm{c} 1.148, \mathrm{CH}_{2} \mathrm{Cl}_{2}\right)$

\section{Compound 10}

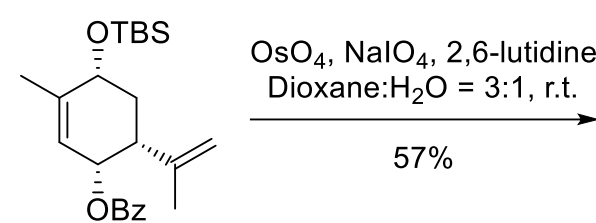

9<smiles>CC(=O)O[C@H]1C=C(C)[C@@H]([SeH])C[C@H]1C(C)=O</smiles>

10

To a compound, $9(1.839 \mathrm{~g}, 4.76 \mathrm{mmol})$ in dioxane $(75 \mathrm{~mL})$ and $\mathrm{H}_{2} \mathrm{O}(25 \mathrm{~mL})$ was added 2,6-lutidine $(1.11 \mathrm{~mL}, 9.51 \mathrm{mmol}), \mathrm{OsO}_{4}(2.5 \mathrm{wt} \%$ in tBuOH$, 967.6 \mu \mathrm{L}, 0.095 \mathrm{mmol})$ and sodium periodate $(4.07 \mathrm{~g}$, $19.03 \mathrm{mmol}$ ). The mixture was stirred for $1 \mathrm{~h}$ before being diluted with $\mathrm{H}_{2} \mathrm{O}$ and $\mathrm{Na}_{2} \mathrm{~S}_{2} \mathrm{O}_{3}$. After $30 \mathrm{~min}$, organic layer was extracted with DCM. The combined organic layers were dried over $\mathrm{MgSO}_{4}$, filtered, and concentrated under reduced pressure. The residue was purified by column chromatography on silica gel (EtOAc : Hexane $=1: 15$ to 1:10) to give $\mathbf{1 0}(1.05 \mathrm{~g}, 57 \%$, crystalline solid).

${ }^{1}$ H NMR (400 MHz, Chloroform-d) $\delta 7.96-7.89(\mathrm{~m}, 2 \mathrm{H}), 7.52(\mathrm{td}, J=7.5,1.3 \mathrm{~Hz}, 1 \mathrm{H}), 7.43-7.34$ $(\mathrm{m}, 2 \mathrm{H}), 5.78(\mathrm{~d}, J=1.3 \mathrm{~Hz}, 2 \mathrm{H}), 4.11(\mathrm{dd}, J=10.7,5.6 \mathrm{~Hz}, 1 \mathrm{H}), 2.79(\mathrm{dt}, J=13.2,3.0 \mathrm{~Hz}, 1 \mathrm{H}), 2.24$ $-2.17(\mathrm{~m}, 1 \mathrm{H}), 2.15(\mathrm{~s}, 3 \mathrm{H}), 2.01$ (td, $J=13.1,10.7 \mathrm{~Hz}, 1 \mathrm{H}), 1.76(\mathrm{~s}, 3 \mathrm{H}), 0.90(\mathrm{~s}, 9 \mathrm{H}), 0.12(\mathrm{~s}, 3 \mathrm{H})$, $0.09(\mathrm{~s}, 3 \mathrm{H})$

${ }^{13}$ C NMR (101 MHz, Chloroform- $d$ ) $\delta$ 206.2, 166.2, $146.8,133.4,131.0,130.0,128.6,119.7,70.6$, $67.8,50.4,29.3,27.9,26.0,19.9,18.3,-3.9,-4.7$.

High Resolution MS (ESI-TOF) m/z: [M+Na] $]^{+}$Calcd for $\mathrm{C}_{22} \mathrm{H}_{32} \mathrm{O}_{4} \mathrm{SiNa} 411.1968$; Found: 411.1975 $[\alpha]^{25}=+41.9923\left(\mathrm{c} 1.1645, \mathrm{CH}_{2} \mathrm{Cl}_{2}\right)$

\section{Compound 11}<smiles>CC(=O)O[C@H]1C=C(C)[C@@H](O[SbH3])[C@H](C(C)=O)C1</smiles>

10

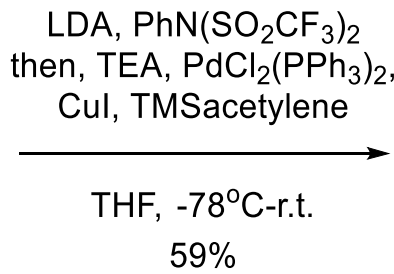

$59 \%$

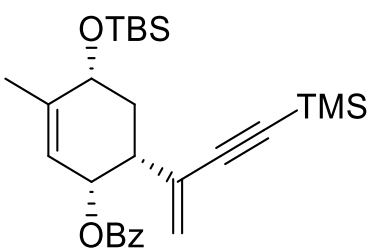

11

To a solution of diisopropylamine (DIPA; $412.5 \mu \mathrm{L}, 2.94 \mathrm{mmol})$ in THF $(22 \mathrm{~mL})$ was added $\mathrm{n}$ butyllithium $(2.467 \mathrm{M}$ in hexane, $1.181 \mathrm{~mL}, 2.94 \mathrm{mmol})$ dropwise at $0{ }^{\circ} \mathrm{C}$. After stirring for $10 \mathrm{~min}$, the reaction mixture was cooled to $-78^{\circ} \mathrm{C}$. To this reaction mixture, was added ketone compound $(879.97$ 
$\mathrm{mg}, 2.264 \mathrm{mmol})$ in THF $(2 \mathrm{~mL})$ via cannula at $-78{ }^{\circ} \mathrm{C}$. After stirring for $1 \mathrm{~h}, \mathrm{~N}-$ phenyltrimethylsulfoneimide $(1.618 \mathrm{~g}, 4.53 \mathrm{mmol})$ was added dropwise at $-78^{\circ} \mathrm{C}$. After stirring for 30 min. at $-78{ }^{\circ} \mathrm{C}$, the mixture was allowed to warm up to $0{ }^{\circ} \mathrm{C}$ and stirred for $1 \mathrm{~h}$. Them after warm up to r.t., TEA (5.83 mL, $41.78 \mathrm{mmol}), \mathrm{PdCl}_{2}\left(\mathrm{PPh}_{3}\right)$ (39.097 mg, mmol), CuI (26.52 mg, mmol) and trimethylsilylacetylene $(472.43 \mu \mathrm{L}, \mathrm{mmol})$ were added to the reaction mixture. After $10 \mathrm{~min}$., the reaction mixture was quenched with brine, and the organic layer was separated and extracted with $\mathrm{Et}_{2} \mathrm{O}$. The combined organic layer was dried over $\mathrm{MgSO}_{4}$, filtered, and concentrated under reduced pressure. The residue was purified by column chromatography on silica gel (EtOAc : Hexane $=1: 50$ ) to give 11 (626.18 mg, 59\% / if it is carried out in 2 steps, $77 \%$ yield of product was gained, pale yellow oil)

${ }^{1} \mathbf{H}$ NMR (400 MHz, Chloroform- $d$ ) $\delta 8.04-7.97(\mathrm{~m}, 2 \mathrm{H}), 7.54-7.46(\mathrm{~m}, 1 \mathrm{H}), 7.38(\mathrm{t}, J=7.7 \mathrm{~Hz}$, $2 \mathrm{H}), 5.82(\mathrm{dd}, J=3.7,1.7 \mathrm{~Hz}, 1 \mathrm{H}), 5.53(\mathrm{~s}, 1 \mathrm{H}), 5.50-5.45(\mathrm{~m}, 1 \mathrm{H}), 5.38(\mathrm{~s}, 1 \mathrm{H}), 4.21-4.15(\mathrm{~m}$, $1 \mathrm{H}), 2.59(\mathrm{~d}, J=12.0 \mathrm{~Hz}, 1 \mathrm{H}), 2.12-2.00(\mathrm{~m}, 2 \mathrm{H}), 1.76(\mathrm{~s}, 3 \mathrm{H}), 0.92(\mathrm{~s}, 9 \mathrm{H}), 0.13-0.10(\mathrm{~m}, 15 \mathrm{H})$; ${ }^{13}$ C NMR (101 MHz, Chloroform- $d$ ) $\delta 166.0,144.9,132.6,131.6,130.7,129.7,128.1,122.5,120.8$, 104.3, 95.1, 71.0, 68.9, 42.0, 32.1, 25.8, 25.6, 19.6, 18.1, -0.2, -4.2, -4.9.

High Resolution MS (ESI-TOF) m/z: [M+Na] ${ }^{+}$Calcd for $\mathrm{C}_{27} \mathrm{H}_{40} \mathrm{O}_{3} \mathrm{Si}_{2} \mathrm{Na}$ 491.2414; Found:491.2427 $[\alpha]^{25}=-163.6551\left(\mathrm{c} 0.8910, \mathrm{CH}_{2} \mathrm{Cl}_{2}\right)$

\section{Compound 12}

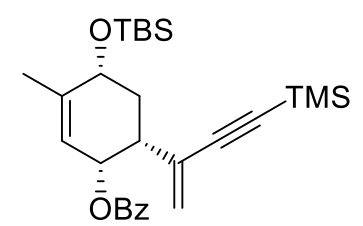

11

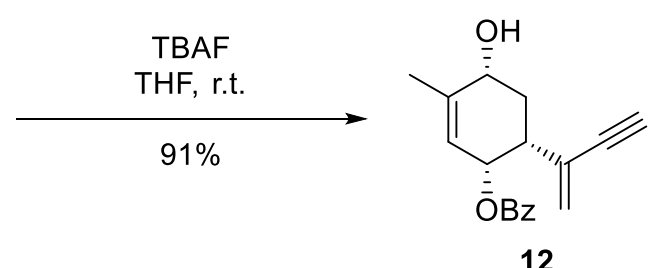

12

To a solution of $\mathbf{1 1}(812.26 \mathrm{mg}, 1.73 \mathrm{mmol})$ in THF $(30 \mathrm{~mL})$ was added tetra-n-butylammonium fluoride (TBAF; $5.20 \mathrm{~mL}$ of $1.0 \mathrm{M}$ in THF, $5.19 \mathrm{mmol}$ ) and stirred for $1 \mathrm{~h}$. The reaction mixture was quenched with saturated $\mathrm{NH}_{4} \mathrm{Cl}$, and the organic layer was separated and extracted with $\mathrm{Et}_{2} \mathrm{O}$. The combined organic layer was dried over $\mathrm{MgSO}_{4}$, filtered, and concentrated under reduced pressure. The residue was purified by column chromatography on silica gel (EtOAc : Hexane $=1: 5$ ) to give 12 (444.48 mg, 91\%, colorless oil).

${ }^{1}$ H NMR (400 MHz, Chloroform- $d$ ) $\delta 8.01-7.96(\mathrm{~m}, 2 \mathrm{H}), 7.54-7.47(\mathrm{~m}, 1 \mathrm{H}), 7.42-7.34(\mathrm{~m}, 2 \mathrm{H})$, $5.86(\mathrm{dt}, J=5.3,1.5 \mathrm{~Hz}, 1 \mathrm{H}), 5.60-5.54(\mathrm{~m}, 2 \mathrm{H}), 5.44(\mathrm{t}, J=0.9 \mathrm{~Hz}, 1 \mathrm{H}), 4.20(\mathrm{ddd}, J=7.7,6.0,3.0$ $\mathrm{Hz}, 1 \mathrm{H}), 2.86(\mathrm{~s}, 1 \mathrm{H}), 2.63(\mathrm{dt}, J=14.5,2.8 \mathrm{~Hz}, 1 \mathrm{H}), 2.25$ (dddd, $J=9.4,7.4,2.7,1.4 \mathrm{~Hz}, 1 \mathrm{H}), 2.03$ (ddd, $J=13.1,12.2,10.3 \mathrm{~Hz}, 1 \mathrm{H}), 1.83(\mathrm{~d}, J=1.2 \mathrm{~Hz}, 3 \mathrm{H})$

${ }^{13}$ C NMR (101 MHz, Chloroform- $d$ ) $\delta$ 166.0, 143.9, 132.8, 130.4, 130.4, 129.6, 128.2, 123.4, 121.4, 82.9, 78.0, 70.2, 68.4, 42.3, 31.5, 18.9 .

High Resolution MS (ESI-TOF) m/z: [M+Na] ${ }^{+} \mathrm{Calcd}$ for $\mathrm{C}_{18} \mathrm{H}_{18} \mathrm{O}_{3} \mathrm{Na}$ 305.1154; Found: 305.1130 $[\alpha]^{25}=-311.0170\left(\mathrm{c} 0.59, \mathrm{CH}_{2} \mathrm{Cl}_{2}\right)$ 


\section{Compound 13}<smiles>C#CC(=C)[C@H]1C[C@H](O)C(C)=C[C@H]1OC(=O)O</smiles>

12

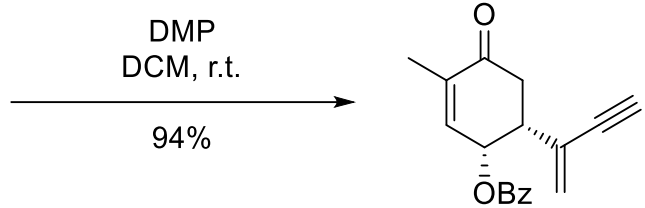

13

To a solution of the alcohol compound, $12(356.36 \mathrm{mg}, 1.262 \mathrm{mmol})$ in DCM (10 mL) was added Dess-Martin periodinane (DMP; $642.42 \mathrm{mg}, 1.51 \mathrm{mmol}$ ) at $0{ }^{\circ} \mathrm{C}$ and then allowed warm to room temperature. After stirring for $1 \mathrm{~h}$, the reaction mixture was quenched with saturated $\mathrm{Na}_{2} \mathrm{~S}_{2} \mathrm{O}_{3}$, and the organic layer was separated and extracted with DCM. The combined organic layer was dried over $\mathrm{MgSO}_{4}$, filtered, and concentrated under reduced pressure. The residue was purified by column chromatography on silica gel (EtOAc : Hexane $=1: 20)$ to give $\mathbf{1 3}(321.93 \mathrm{~g}, 91 \%$, crystalline solid).

${ }^{1}$ H NMR (400 MHz, Benzene- $\left.d_{6}\right) \delta 8.16(\mathrm{~d}, J=9.7 \mathrm{~Hz}, 2 \mathrm{H}), 7.20-7.14(\mathrm{~m}, 1 \mathrm{H}), 7.14-7.05(\mathrm{~m}, 2 \mathrm{H})$, $6.56(\mathrm{~d}, J=6.7 \mathrm{~Hz}, 1 \mathrm{H}), 5.82-5.74(\mathrm{~m}, 1 \mathrm{H}), 5.43(\mathrm{~s}, 1 \mathrm{H}), 5.04(\mathrm{~s}, 2 \mathrm{H}), 2.87$ (dd, $J=16.3,11.7 \mathrm{~Hz}$, $1 \mathrm{H}), 2.64(\mathrm{dd}, J=12.3,3.2 \mathrm{~Hz}, 1 \mathrm{H}), 2.57(\mathrm{~s}, 1 \mathrm{H}), 2.46(\mathrm{dd}, J=16.7,4.5 \mathrm{~Hz}, 1 \mathrm{H}), 1.73(\mathrm{~s}, 3 \mathrm{H})$ ${ }^{13}$ C NMR (101 MHz, Benzene- $\left.d_{6}\right) \delta 196.3,165.6,138.9,138.2,133.1,130.5,130.0,129.8,128.5,124.4$, 82.5, 79.3, 68.1, 43.4, 37.7, 15.7 .

High Resolution MS (ESI-TOF) m/z: [M+Na] ${ }^{+}$Calcd for $\mathrm{C}_{18} \mathrm{H}_{16} \mathrm{O}_{3} \mathrm{Na}$ 303.0997; Found: 303.0970 $[\alpha]^{25} \mathrm{D}=-264.3359\left(\mathrm{c} 0.5220, \mathrm{CH}_{2} \mathrm{Cl}_{2}\right)$

\section{Compound 14}<smiles>C#CC(=C)[C@H]1CC(=O)C(C)=C[C@H]1OC(=O)O</smiles>

13

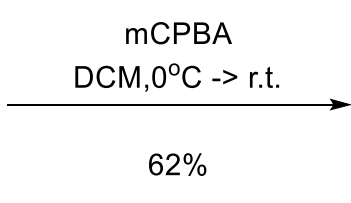

$62 \%$<smiles>C#CC1([C@@H]2CC(=O)C(C)=C[C@H]2OC(=O)c2ccccc2)CO1</smiles>

14

To a solution of ene-yne compound $13(84.9 \mathrm{mg}, 0.303 \mathrm{mmol})$ in DCM $(\mathrm{mL})$ cooled to $0{ }^{\circ} \mathrm{C}$ was added meta-chloroperbenzoic acid(mCPBA, $\leq 77 \% ; 1.11 \mathrm{~g}, 4.955 \mathrm{mmol})$. then warmed to room temperature slowly. The resulting was quenched with saturated $\mathrm{NaHCO}_{3}$ solution and extracted with DCM. The combined organic layers were dried over $\mathrm{MgSO}_{4}$, filtered and concentrated under reduced pressure. The residue was purified by column chromatography on silica gel (EtOAc : Hexane $=1: 20$ ) to afford diastereomeric mixture of $\mathbf{1 4}(55.67 \mathrm{mg}, 62 \%$, dr $1.8: 1$, crystalline solid).

High Resolution MS (ESI-TOF) m/z: [M+Na] ${ }^{+}$Calcd for $\mathrm{C}_{18} \mathrm{H}_{16} \mathrm{O}_{4} \mathrm{Na} 319.0946$; Found: 319.0925 


\section{Compound 17}

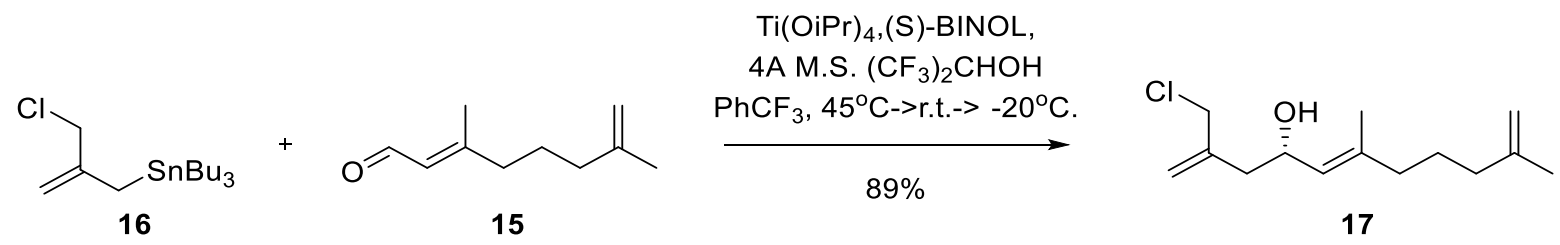

A flame-dried round bottom flask that contained (S)-BINOL (252 $\mathrm{mg}, 0.88 \mathrm{mmol})$ and activated powdered 4-A molecular sieves $(600 \mathrm{mg}$ ) was evacuated, carefully purged with argon, and then charged with dry $\mathrm{PhCF}_{3}(2.0 \mathrm{~mL})$ followed by $\operatorname{Ti}(\mathrm{O} i \mathrm{Pr})(257 \mu \mathrm{L}, 0.88 \mathrm{mmol})$. The mixture was allowed to proceed at $45^{\circ} \mathrm{C}$ in oil bath for $2 \mathrm{~h}$. After cooling to $25^{\circ} \mathrm{C}, 1,1,1,3,3,3$,-hexafluoro-2-propanol $(185 \mu \mathrm{L}$, $1.76 \mathrm{mmol}$ ) wa added. The resulting solution was warmed to $45^{\circ} \mathrm{C}$ in oil bath for $2 \mathrm{~h}$. The solution was cooled to $25^{\circ} \mathrm{C}$ and concentrated under vacuum (Schlenk line) and then purged with argon. A solution of Fragemnt $15^{1}(67 \mathrm{mg}, 0.44 \mathrm{mmol})$ in dry $\mathrm{PhCF}_{3}(1.0 \mathrm{~mL})$ was added to this reaction mixture at $25^{\circ} \mathrm{C}$. The solution was cooled to $-20^{\circ} \mathrm{C}$ (Eyela PSL-1810, constant temperature bath with magnetic stirrer), allyl tin compound, $\mathbf{1 6}^{2}$ (333.44 $\mathrm{mg}, 0.88 \mathrm{mmol}$ ) in dry $\mathrm{PhCF}_{3}(1.0 \mathrm{~mL})$ was added dropwise through cannula. The reaction was kept at $-20^{\circ} \mathrm{C}$, and after $24 \mathrm{~h}$, additional allyltin compound $(167 \mathrm{mg}, 0.44$ $\mathrm{mmoL})$ in dry $\mathrm{PhCF}_{3}(1.0 \mathrm{~mL})$ was added via cannula. After total 3days at $-20^{\circ} \mathrm{C}$, reaction mixture was qeunched with saturated aqueous $\mathrm{NaHCO}_{3}(5.0 \mathrm{~mL})$ and diluted with DCM $(5.0 \mathrm{~mL})$. The molecular sieves were removed by filtration, and the aqueous layer was extracted with DCM $(5.0 \mathrm{~mL})$ for three times. The combined organic layers were dried over $\mathrm{MgSO}_{4}$, filtered and concentrated under reduced pressure, then residue was purified by column chromatography on silica gel (EtOAc: Hexane $=1: 5$ to $1: 3$ ) to give compound 17 . (95.10 mg, 89\%, yellowish oil).

${ }^{1}$ H NMR (400 MHz, Chloroform- $d$ ) $\delta 5.23(\mathrm{~s}, 1 \mathrm{H}), 5.19$ (dq, $\left.J=8.5,1.3 \mathrm{~Hz}, 1 \mathrm{H}\right), 5.06$ (d, $J=1.2 \mathrm{~Hz}$ $1 \mathrm{H}), 4.69(\mathrm{~s}, 1 \mathrm{H}), 4.65(\mathrm{~d}, J=1.2 \mathrm{~Hz}, 1 \mathrm{H}), 4.56(\mathrm{q}, J=7.5 \mathrm{~Hz}, 1 \mathrm{H}), 4.14-4.04(\mathrm{~m}, 2 \mathrm{H}), 2.41-2.34(\mathrm{~m}$, 2H), 2.01-1.90 (m, 4H), 1.73-1.63 (m, 6H), 1.61-1.44 (m, 2H);

${ }^{13}$ C NMR (101 MHz, Chloroform- $d$ ) $\delta 145.71,141.92,139.19,126.95,117.39,109.95,66.76$, $48.36,41.35,39.00,37.29,25.52,22.39,16.59$

High Resolution MS (ESI-TOF) m/z: [M+Na] $]^{+}$Calcd for $\mathrm{C}_{14} \mathrm{H}_{23} \mathrm{ClONa} 265.1335$; Found: 265.1316 

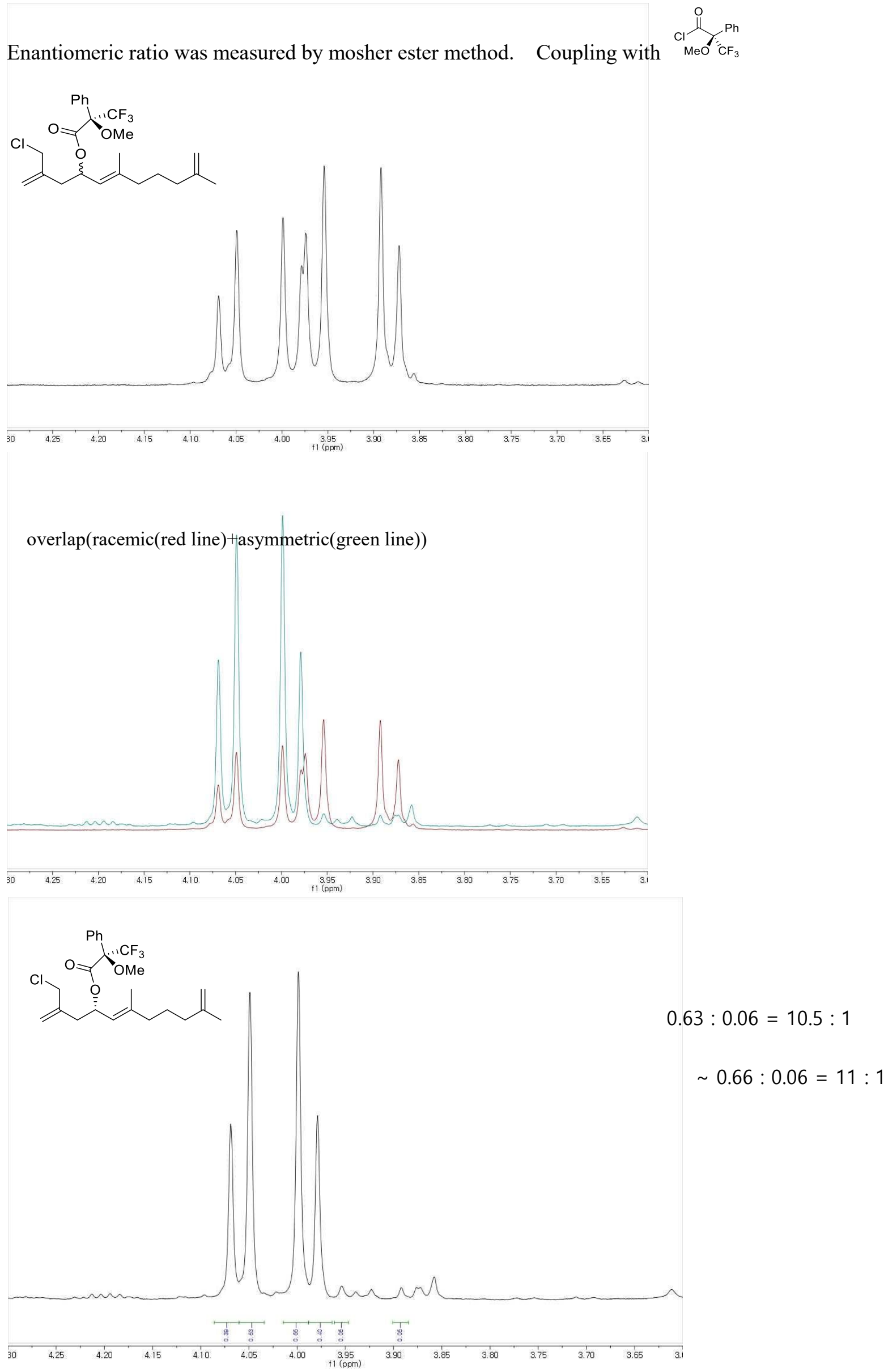


\section{Compound 18}<smiles>C#CC(C)(C)C1CC(=O)C(C)=CC1OCC</smiles>

14

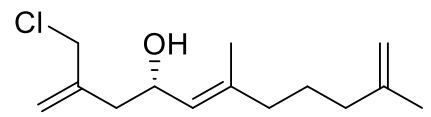

17

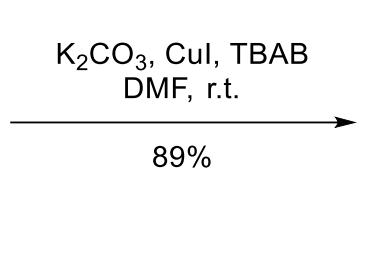

To a stirred solution of diasteromeric mixture of enone compound 14 (25.01 $\mathrm{mg}, 0.084 \mathrm{mmol})$ in DMF $(0.5 \mathrm{~mL})$ were added $\mathrm{K}_{2} \mathrm{CO}_{3}(17.50 \mathrm{mg}, 0.13 \mathrm{mmol})$, tetrabutylammonium bromide (TBAB; $54.42 \mathrm{mg}$, $0.17 \mathrm{mmol})$ and $\mathrm{CuI}(16.07 \mathrm{mg}, 0.13 \mathrm{mmol})$. Then allylic alochol 17(24.6 mg, $0.1 \mathrm{mmol})$ in DMF (1.0 $\mathrm{mL}$ ) was added via cannula. The resulting mixture was stirred for $36 \mathrm{~h}$. The resulting mixture was diluted with $\mathrm{Et}_{2} \mathrm{O}$ and quenched with $\mathrm{H}_{2} \mathrm{O}$ and brine. The resulting mixture was extracted with $\mathrm{Et}_{2} \mathrm{O}$, and then combined organic layers were dried over $\mathrm{MgSO}_{4}$, filtered, and concentrated under reduced pressure. The residue was purified by column chromatography on silica gel (EtOAc : Hexane $=1: 20$ ) to afford 18 (37.79 mg, 89\%, dr 1.8:1, pale yellow oil).

High Resolution MS (ESI-TOF) m/z: [M+Na] ${ }^{+}$Calcd for $\mathrm{C}_{32} \mathrm{H}_{38} \mathrm{O}_{5} \mathrm{Na}$ 525.2617; Found: 525.2620

\section{Compound 19}
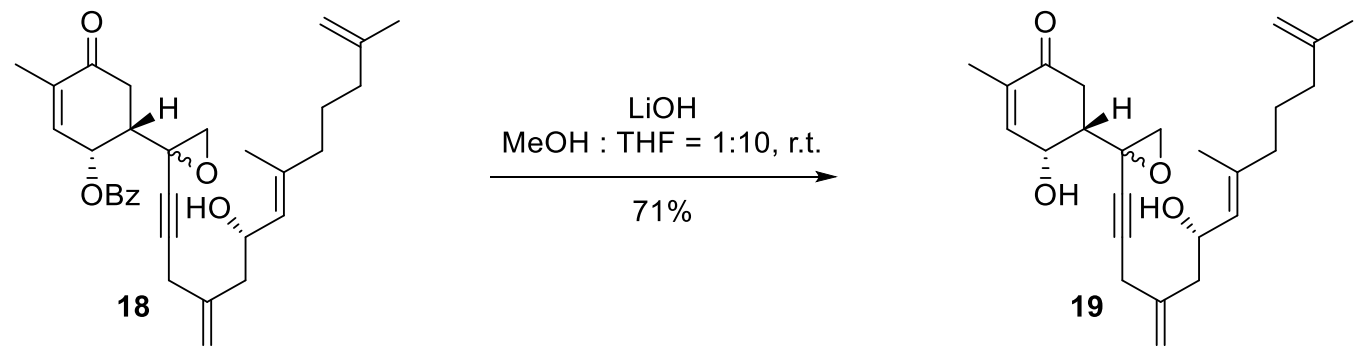

To a stirred solution of $18(24.8 \mathrm{mg}, 0.049 \mathrm{mmol})$ in $\mathrm{MeOH}(0.1 \mathrm{~mL})$ and THF $(1 \mathrm{~mL})$ was added $\mathrm{LiOH}(1.77 \mathrm{mg}, 0.074 \mathrm{mmol})$. The resulting mixture was stirred for $4 \mathrm{~h}$. The resulting mixture was diluted with $\mathrm{Et}_{2} \mathrm{O}$ and quenched with saturated $\mathrm{NaHCO}_{3}$ solution. The organic layers were extracted with $\mathrm{Et}_{2} \mathrm{O}$ and combined organic layers were dried over $\mathrm{MgSO}_{4}$, filtered, and concentrated under reduced pressure. The residue was purified by column chromatography on silica gel (EtOAc : Hexane $=1: 2$ ) to afford 19 in diastereomeric mixture (13.94 mg, 71\%, pale yellow oil). NMR was measured with sligltly separted major and minor compound of $\mathbf{1 9 .}$

Major : ${ }^{1}$ H NMR $(599 \mathrm{MHz}$, Chloroform- $d$ ) $\delta 6.72(\mathrm{~d}, J=5.4 \mathrm{~Hz}, 1 \mathrm{H}), 5.17(\mathrm{~d}, J=8.3 \mathrm{~Hz}, 1 \mathrm{H}), 5.12$ (s, 1H), $4.95(\mathrm{~s}, 1 \mathrm{H}), 4.72(\mathrm{~s}, 1 \mathrm{H}), 4.69(\mathrm{~s}, 1 \mathrm{H}), 4.65(\mathrm{~s}, 1 \mathrm{H}), 4.55-4.49(\mathrm{~m}, 1 \mathrm{H}), 3.02(\mathrm{~s}, 2 \mathrm{H}), 2.97(\mathrm{~d}$, $J=5.1 \mathrm{~Hz}, 1 \mathrm{H}), 2.92(\mathrm{~d}, J=5.1 \mathrm{~Hz}, 1 \mathrm{H}), 2.76(\mathrm{dd}, J=16.1,11.5 \mathrm{~Hz}, 1 \mathrm{H}), 2.40(\mathrm{dd}, J=11.7,3.7 \mathrm{~Hz}$, $1 \mathrm{H}), 2.36(\mathrm{dd}, J=16.3,4.2 \mathrm{~Hz}, 1 \mathrm{H}), 2.31(\mathrm{dd}, J=14.2,8.2 \mathrm{~Hz}, 1 \mathrm{H}), 2.23(\mathrm{dd}, J=14.4,5.0 \mathrm{~Hz}, 1 \mathrm{H})$, $1.96(\mathrm{q}, J=7.2 \mathrm{~Hz}, 4 \mathrm{H}), 1.79$ (s, 3H), 1.69 (s, 3H), 1.67 (s, 3H), 1.52 (td, $J=7.7,3.8 \mathrm{~Hz}, 2 \mathrm{H})$

${ }^{13}$ C NMR (151 MHz, Chloroform- $d$ ) $\delta$ 198.0, 145.7, 142.1, 140.2, 139.3, 137.4, 127.0, 114.7, 110.0, 82.9, 79.9, 66.8, 65.1, 51.6, 51.4, 43.9, 43.4, 39.0, 37.3, 34.3, 26.4, 25.6, 22.4, 16.6, 15.6 . 
High Resolution MS (ESI-TOF) m/z: [M+Na] ${ }^{+}$Calcd for $\mathrm{C}_{25} \mathrm{H}_{34} \mathrm{O}_{4} \mathrm{Na} 421.2355$; Found: 421.2360

Minor : ${ }^{1} \mathbf{H}$ NMR $(599 \mathrm{MHz}$, Chloroform- $d$ ) $\delta 6.71(\mathrm{~d}, J=5.6 \mathrm{~Hz}, 1 \mathrm{H}), 5.17(\mathrm{~d}, J=8.3 \mathrm{~Hz}, 1 \mathrm{H}), 5.12$ (s, 1H), $4.95(\mathrm{~s}, 1 \mathrm{H}), 4.69(\mathrm{~s}, 1 \mathrm{H}), 4.65(\mathrm{~s}, 1 \mathrm{H}), 4.61(\mathrm{~s}, 1 \mathrm{H}), 4.55-4.48(\mathrm{~m}, 1 \mathrm{H}), 3.05(\mathrm{~d}, J=5.1 \mathrm{~Hz}$, $1 \mathrm{H}), 3.01(\mathrm{~s}, 2 \mathrm{H}), 2.95(\mathrm{~d}, J=5.1 \mathrm{~Hz}, 1 \mathrm{H}), 2.79-2.70(\mathrm{~m}, 1 \mathrm{H}), 2.49(\mathrm{dd}, J=16.4,3.8 \mathrm{~Hz}, 1 \mathrm{H}), 2.33-$ $2.28(\mathrm{~m}, 2 \mathrm{H}), 2.23(\mathrm{dd}, J=14.1,4.7 \mathrm{~Hz}, 1 \mathrm{H}), 1.97(\mathrm{q}, J=7.1 \mathrm{~Hz}, 4 \mathrm{H}), 1.79(\mathrm{~s}, 3 \mathrm{H}), 1.69(\mathrm{~s}, 3 \mathrm{H}), 1.67$ $(\mathrm{s}, 3 \mathrm{H}), 1.53(\mathrm{td}, J=7.7,3.4 \mathrm{~Hz}, 2 \mathrm{H})$;

${ }^{13}$ C NMR (151 MHz, Chloroform- $d$ ) $\delta$ 198.1, 145.7, 141.7, 140.1, 139.3, 137.5, 127.0, 114.8, 110.0, 83.2, 79.6, 66.8, 64.3, 53.6, 51.5, 43.9, 43.4, 39.0, 37.3, 34.7, 26.5, 25.6, 22.4, 16.6, 15.7.

High Resolution MS (ESI-TOF) $\mathbf{m} / \mathbf{z}:[\mathrm{M}+\mathrm{Na}]^{+}$Calcd for $\mathrm{C}_{25} \mathrm{H}_{34} \mathrm{O}_{4} \mathrm{Na} 421.2355$; Found: 421.2357

\section{Compound 1}
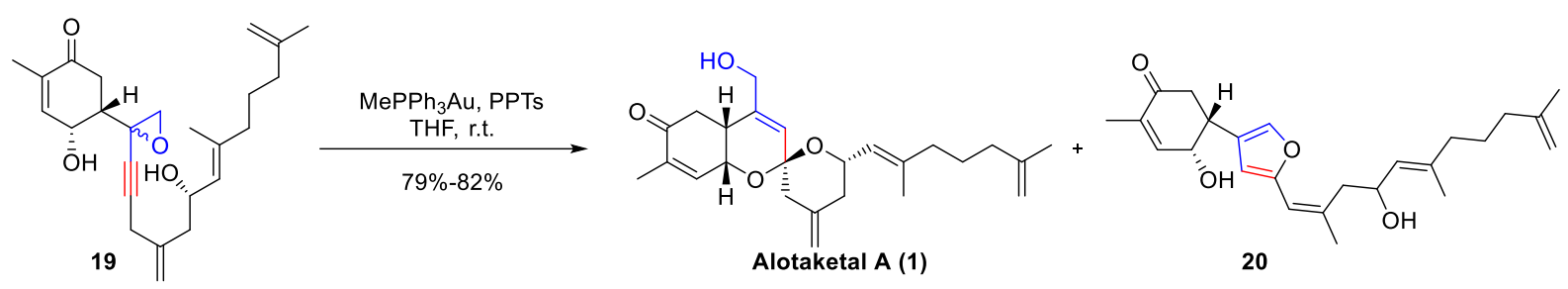

- $\quad 1$ eq. of $\mathrm{PPh}_{3} \mathrm{AuMe}$

To a stirred solution of $\mathrm{MePPh}_{3} \mathrm{Au}(11.20 \mathrm{mg}, 0.024 \mathrm{mmol})$ and PPTs $(5.93 \mathrm{mg}, 0.024 \mathrm{mmol})$ in THF $(1 \mathrm{~mL})$ was added diol compound $19(9.41 \mathrm{mg}, 0.024 \mathrm{mmol})$ in THF $(1 \mathrm{~mL})$. After 30min, the resulting mixture was diluted with $\mathrm{Et}_{2} \mathrm{O}$ and quenched with saturated $\mathrm{NaHCO}_{3}$ solution. The organic layers were extracted with $\mathrm{Et}_{2} \mathrm{O}$ and combined organic layers were dried over $\mathrm{MgSO}_{4}$, filtered, and concentrated under reduced pressure. The residue was purified by column chromatography on silica gel (EtOAc : Hexane $=1: 3$ to $1: 2)$ to give alotaketal A $(7.44 \mathrm{mg}, 79 \%$, amorphous solid). The ratio of alotaketal $\mathrm{A}(\mathbf{1})$ and 20 was about $25: 1$, measured in $400 \mathrm{MHz}$ NMR with silica- filtered- product mixture.

\section{- $\quad 0.1$ eq. of $\mathrm{PPh}_{3} \mathrm{AuMe}$}

To a stirred solution of $\mathrm{MePPh}_{3} \mathrm{Au}(1.21 \mathrm{mg}, 0.0025 \mathrm{mmol})$ and PPTs $(6.39 \mathrm{mg}, 0.025 \mathrm{mmol})$ in THF $(1 \mathrm{~mL})$ was added diol compound $19(10.13 \mathrm{mg}, 0.025 \mathrm{mmol})$ in THF $(1 \mathrm{~mL})$. After $2 \mathrm{~h}$, the resulting mixture was diluted with $\mathrm{Et}_{2} \mathrm{O}$ and quenched with saturated $\mathrm{NaHCO}_{3}$ solution. The organic layers were extracted with $\mathrm{Et}_{2} \mathrm{O}$ and combined organic layers were dried over $\mathrm{MgSO}_{4}$, filtered, and concentrated under reduced pressure. The residue was purified by column chromatography on silica gel (EtOAc : Hexane $=1: 3$ to $1: 2)$ to give alotaketal $\mathrm{A}(8.35 \mathrm{mg}, 82 \%$, amorphous solid $)$. The ratio of alotaketal $\mathrm{A}(\mathbf{1})$ and 20 was about $11: 1$, measured in $400 \mathrm{MHz}$ NMR with silica- filtered- product mixture.

${ }^{1}$ H NMR $\left(599 \mathrm{MHz}\right.$, Benzene- $\left.d_{6}\right) \delta 6.33(\mathrm{dd}, J=5.6,1.7 \mathrm{~Hz}, 1 \mathrm{H}), 5.59(\mathrm{~s}, 1 \mathrm{H}), 5.48(\mathrm{dd}, J=8.4,1.3$ $\mathrm{Hz}, 1 \mathrm{H}), 4.89$ (s, 1H), $4.87(\mathrm{~s}, 1 \mathrm{H}), 4.87-4.81(\mathrm{~m}, 1 \mathrm{H}), 4.81(\mathrm{~s}, 1 \mathrm{H}), 4.80(\mathrm{~s}, 1 \mathrm{H}), 4.37(1 \mathrm{H}, \mathrm{dd}, \mathrm{J}=$ 5.6, 3.5 Hz), 3.59 (d, $J=14.2 \mathrm{~Hz}, 1 \mathrm{H}), 3.56$ (d, $J=14.3 \mathrm{~Hz}, 1 \mathrm{H}), 2.49$ (dd, $J=15.7,4.5 \mathrm{~Hz}, 1 \mathrm{H}), 2.41$ $(\mathrm{dd}, J=15.8,13.1 \mathrm{~Hz}, 1 \mathrm{H}), 2.34(\mathrm{~d}, J=13.2 \mathrm{~Hz}, 1 \mathrm{H}), 2.34-2.27(\mathrm{~m}, 1 \mathrm{H}), 2.20(\mathrm{t}, J=12.3 \mathrm{~Hz}, 1 \mathrm{H}), 2.09$ $(\mathrm{dt}, J=13.2,4.1 \mathrm{~Hz}, 1 \mathrm{H}), 1.98(\mathrm{t}, \mathrm{J}=7.5 \mathrm{H}, 2 \mathrm{H}), 1.94(\mathrm{t}, J=7.7 \mathrm{~Hz}, 2 \mathrm{H}), 1.71(\mathrm{~s}, 3 \mathrm{H}), 1.67(\mathrm{~s}, 3 \mathrm{H})$, $1.63(\mathrm{~s}, 3 \mathrm{H}), 1.60-1.51(\mathrm{~m}, 2 \mathrm{H})$; 
${ }^{13}$ C NMR (151 MHz, Benzene- $\left.d_{6}\right) \delta$ 197.6, 145.9, 142.5, 141.5, 139.6, 139.2, 139.1, 126.9, 125.2, 111.4, 110.9, 97.2, 68.6, 63.8, 63.6, 44.1, 40.8, 39.7, 38.6, 38.0, 34.1, 26.4, 22.8, 17.1, 16.4.

High Resolution MS (ESI-TOF) m/z: $[\mathrm{M}+\mathrm{Na}]^{+}$Calcd for $\mathrm{C}_{25} \mathrm{H}_{34} \mathrm{O}_{4} \mathrm{Na}$ 421.2355; Found: 421.2355 $[\alpha]_{\mathrm{D}}^{25}=-56.7404(\mathrm{c} 0.9940, \mathrm{MeOH}) \quad$ lit: $[\alpha]^{25}=-38.9(\mathrm{c} 0.01, \mathrm{MeOH})$ 


\section{Comparison of synthetic NMR data of Alotaketal A with literature}

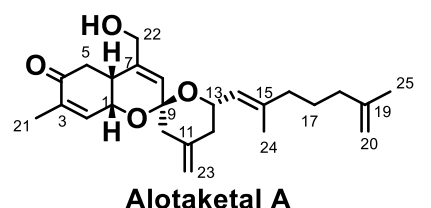

\begin{tabular}{|c|c|c|c|c|}
\hline \multirow[t]{2}{*}{ Position \# } & \multicolumn{2}{|c|}{${ }^{1} \mathrm{H}$ NMR } & \multicolumn{2}{|c|}{${ }^{13} \mathrm{C}$ NMR } \\
\hline & Andersen et al. ${ }^{a}$ & Our group ${ }^{b}$ & Andersen et al. ${ }^{a}$ & Our group ${ }^{c}$ \\
\hline 1 & $4.36(1 \mathrm{H}, \mathrm{dd}, J=4.9,6 \mathrm{~Hz})$ & $4.37(1 \mathrm{H}, \mathrm{dd}, J=5.6,3.5 \mathrm{~Hz})$ & 63.6 & 63.64 \\
\hline 2 & $6.32(1 \mathrm{H}, \mathrm{dq}, J=6,1.2 \mathrm{~Hz})$ & $6.33(1 \mathrm{H}, \mathrm{dd}, J=5.6,1.7 \mathrm{~Hz})$ & 139.6 & 139.63 \\
\hline 3 & & & 139.0 & 139.05 \\
\hline 4 & & & 197.7 & 197.61 \\
\hline $5 a$ & $2.47(1 \mathrm{H}, \mathrm{dd}, J=4.9,15.6 \mathrm{~Hz})$ & $2.49(1 \mathrm{H}, \mathrm{dd}, J=4.5,15.7 \mathrm{~Hz})$ & 38.6 & 38.64 \\
\hline $5 b$ & $2.39(1 \mathrm{H}, \mathrm{dd}, J=13.2,15.6 \mathrm{~Hz})$ & $2.41(1 \mathrm{H}, \mathrm{dd}, J=13.1,15.8 \mathrm{~Hz})$ & 38.6 & 38.64 \\
\hline 6 & $2.06(1 \mathrm{H}, \mathrm{dt}, J=4.9,13.2 \mathrm{~Hz})$ & $2.09(1 \mathrm{H}, \mathrm{dt}, J=4.1,13.2 \mathrm{~Hz})$ & 34.0 & 34.11 \\
\hline 7 & & & 142.5 & 142.50 \\
\hline 8 & $5.56(1 \mathrm{H}, \mathrm{s})$ & $5.59(1 \mathrm{H}, \mathrm{s})$ & 125.1 & 125.22 \\
\hline 9 & & & 97.2 & 97.19 \\
\hline $10 a$ & $2.34(1 \mathrm{H}, \mathrm{d}, J=13.2 \mathrm{~Hz})$ & $2.34(1 \mathrm{H}, \mathrm{d}, J=13.2 \mathrm{~Hz})$ & 44.1 & 44.11 \\
\hline $10 \mathrm{~b}$ & $2.29(1 \mathrm{H}, \mathrm{d}, J=13.2 \mathrm{~Hz})$ & $2.32-2.27(1 \mathrm{H}, \mathrm{m})$ & 44.1 & 44.11 \\
\hline 11 & & & 141.5 & 141.53 \\
\hline $12 a$ & $2.31(1 \mathrm{H}, \mathrm{m})$ & $2.32-2.27(1 \mathrm{H}, \mathrm{m})$ & 40.7 & 40.77 \\
\hline $12 b$ & $2.20(1 \mathrm{H}, \mathrm{t}, J=12.6 \mathrm{~Hz})$ & $2.20(1 \mathrm{H}, \mathrm{t}, J=12.4 \mathrm{~Hz})$ & 40.7 & 40.77 \\
\hline 13 & $4.85(1 \mathrm{H}, \mathrm{ddd}, J=3,8.4,12.6 \mathrm{~Hz})$ & $4.87-4.81(1 \mathrm{H}, \mathrm{m})$ & 68.5 & 68.56 \\
\hline 14 & $5.48(1 \mathrm{H}, \mathrm{d}, J=8.4 \mathrm{~Hz})$ & $5.48(1 \mathrm{H}, \mathrm{dd}, J=8.4,1.3 \mathrm{~Hz})$ & 126.8 & 126.87 \\
\hline 15 & & & 139.2 & 139.22 \\
\hline 16 & $1.98(2 \mathrm{H}, \mathrm{t}, J=7.2 \mathrm{~Hz})$ & $1.98(2 \mathrm{H}, \mathrm{t}, J=7.5 \mathrm{~Hz})$ & 39.7 & 39.71 \\
\hline 17 & $1.55(2 \mathrm{H}, \mathrm{q}, J=7.2 \mathrm{~Hz})$ & $1.60-1.51(2 \mathrm{H}, \mathrm{m})$ & 26.3 & 26.40 \\
\hline 18 & $1.94(2 \mathrm{H}, \mathrm{t}, J=7.2 \mathrm{H})$ & $1.94(2 \mathrm{H}, \mathrm{t}, J=7.7 \mathrm{H})$ & 38.0 & 38.03 \\
\hline 19 & & & 145.9 & 145.88 \\
\hline 20 & $4.80(1 \mathrm{H}, \mathrm{bs}) / 4.81(1 \mathrm{H}, \mathrm{bs})$ & $4.80(1 \mathrm{H}, \mathrm{s}) / 4.81(1 \mathrm{H}, \mathrm{s})$ & 110.9 & 110.91 \\
\hline 21 & $1.71(3 \mathrm{H}, \mathrm{s})$ & $1.71(3 \mathrm{H}, \mathrm{s})$ & 16.4 & 16.39 \\
\hline 22 & $3.53(2 \mathrm{H}, \mathrm{m})$ & $\mathrm{H}, \mathrm{d}, J=14.2 \mathrm{~Hz}), 3.56(1 \mathrm{H}, \mathrm{d}, J=14.3 \mathrm{~Hz})$ & 63.8 & 63.84 \\
\hline $22-\mathrm{OH}$ & $0.53(1 \mathrm{H}, \mathrm{t}, J=5.4 \mathrm{~Hz})$ & & & \\
\hline 23 & $4.87(1 \mathrm{H}, \mathrm{bs}) / 4.89(1 \mathrm{H}, \mathrm{bs})$ & $4.87(1 \mathrm{H}, \mathrm{br} \mathrm{s}) / 4.89(1 \mathrm{H}, \mathrm{br} \mathrm{s})$ & 111.4 & 111.36 \\
\hline 24 & $1.68(3 \mathrm{H}, \mathrm{s})$ & $1.67(3 \mathrm{H}, \mathrm{s})$ & 17.1 & 17.11 \\
\hline 25 & $1.62(3 \mathrm{H}, \mathrm{s})$ & $1.63(3 \mathrm{H}, \mathrm{s})$ & 22.8 & 22.78 \\
\hline
\end{tabular}

a. Forestieri, R.; Merchant, C. E.; de Voogd, N. J.; Matainaho, T.; Kieffer, T. J.; Anderson, R. J. Org. Lett. 2009, 11, 5166

b. Agilent Technologies DD2 $(600 \mathrm{MHz})$, the residual $\mathrm{C}_{6} \mathrm{D}_{6}$ peak $(\delta=7.16)$

c. Agilent Technologies DD2 $(150 \mathrm{MHz})$, the center line of the $\mathrm{C}_{6} \mathrm{D}_{6}$ signal $(128.4 \mathrm{ppm})$ 
4. Comparison of ${ }^{1} \mathrm{H}$ NMR spectrum of synthetic Alotaketal A (600MHz) with literature

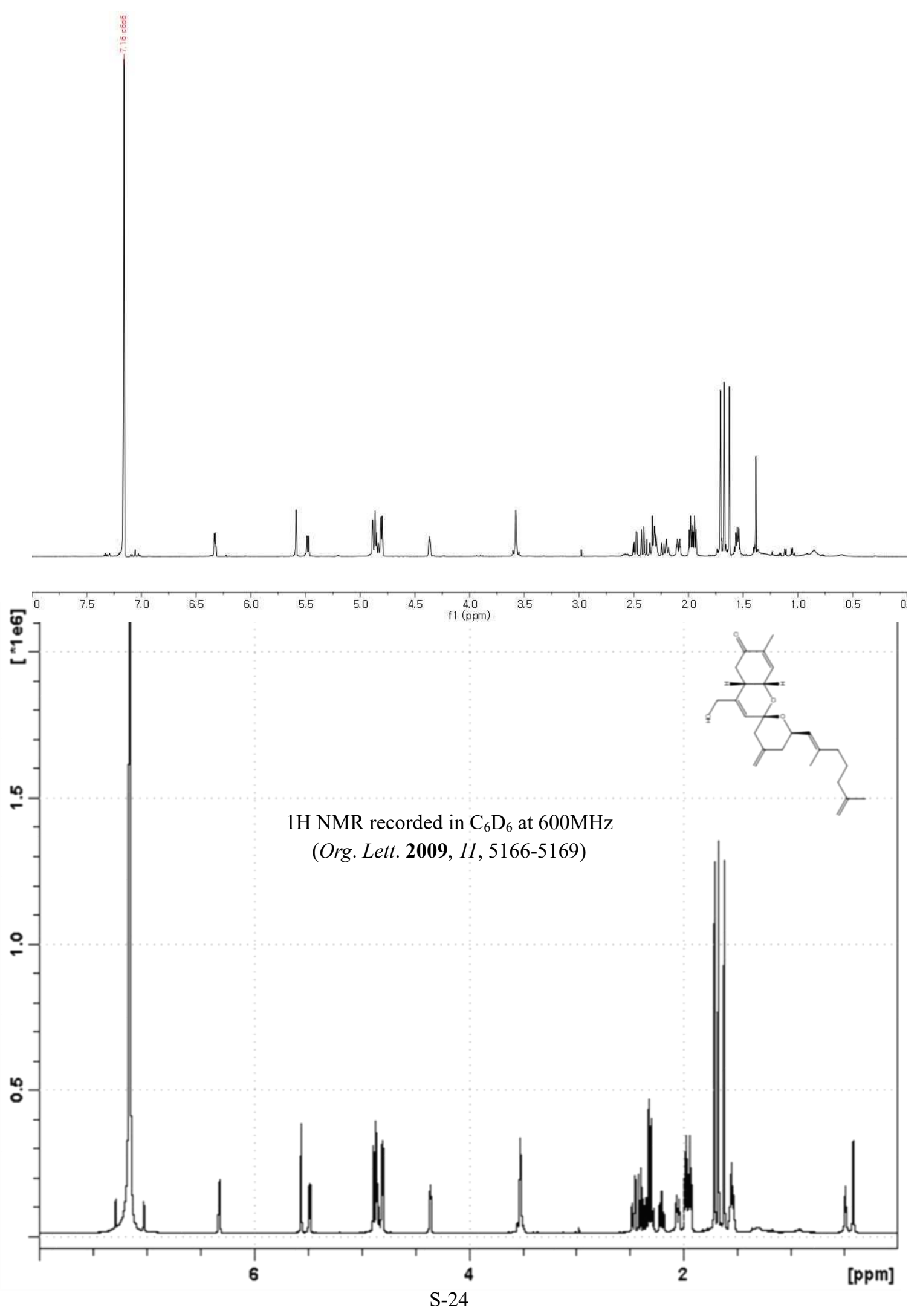




\section{NMR Spectra}
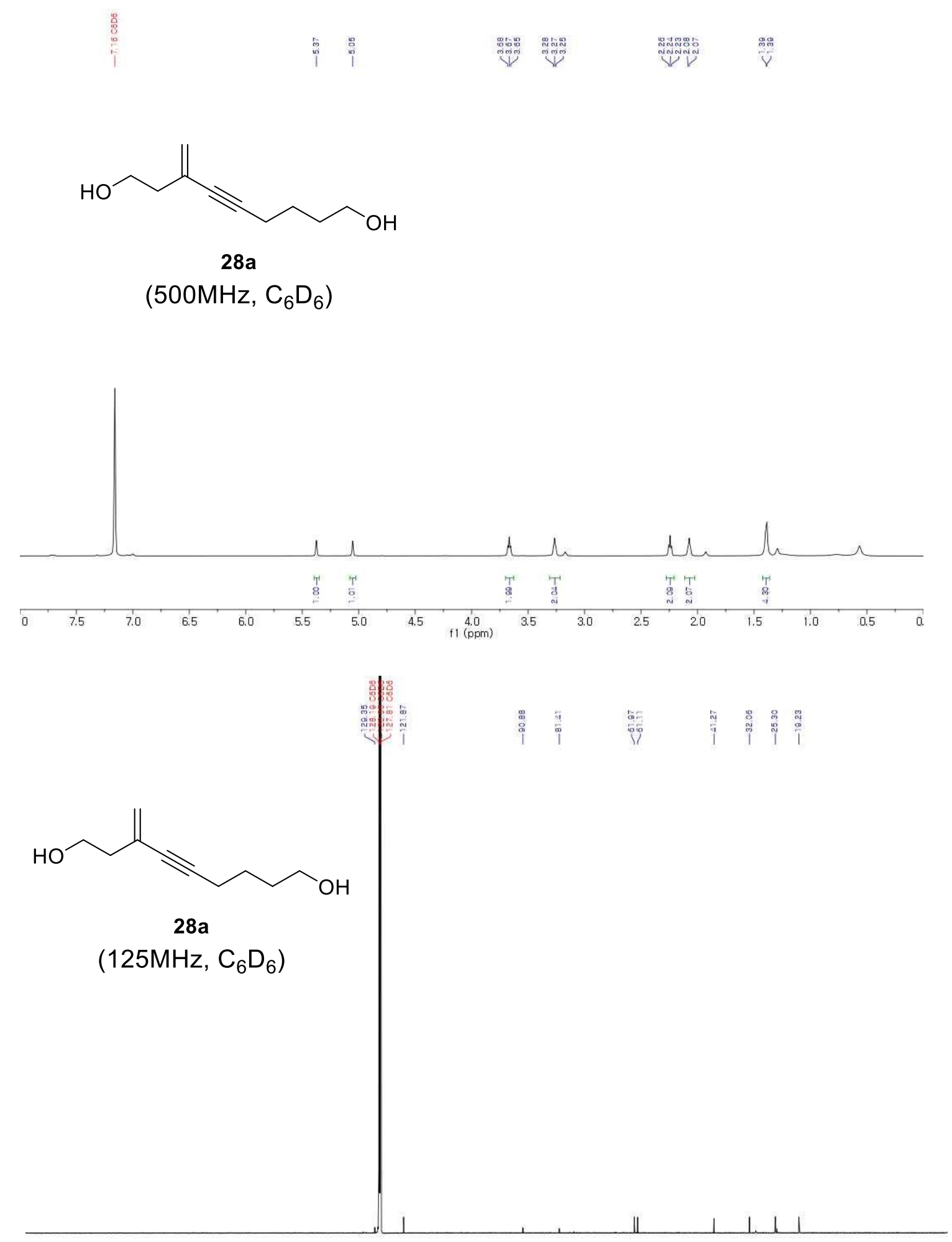

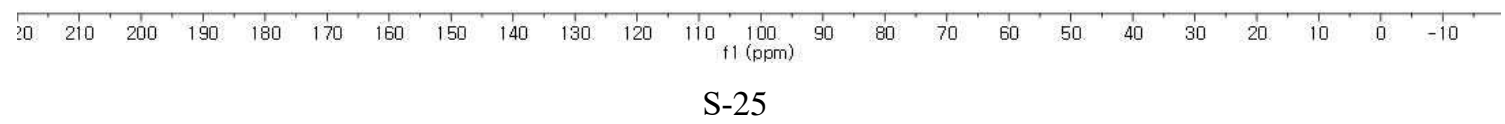


<smiles>C=C(C#CCCO)CCO</smiles>

28b

(400MHz, $\mathrm{C}_{6} \mathrm{D}_{6}$ )
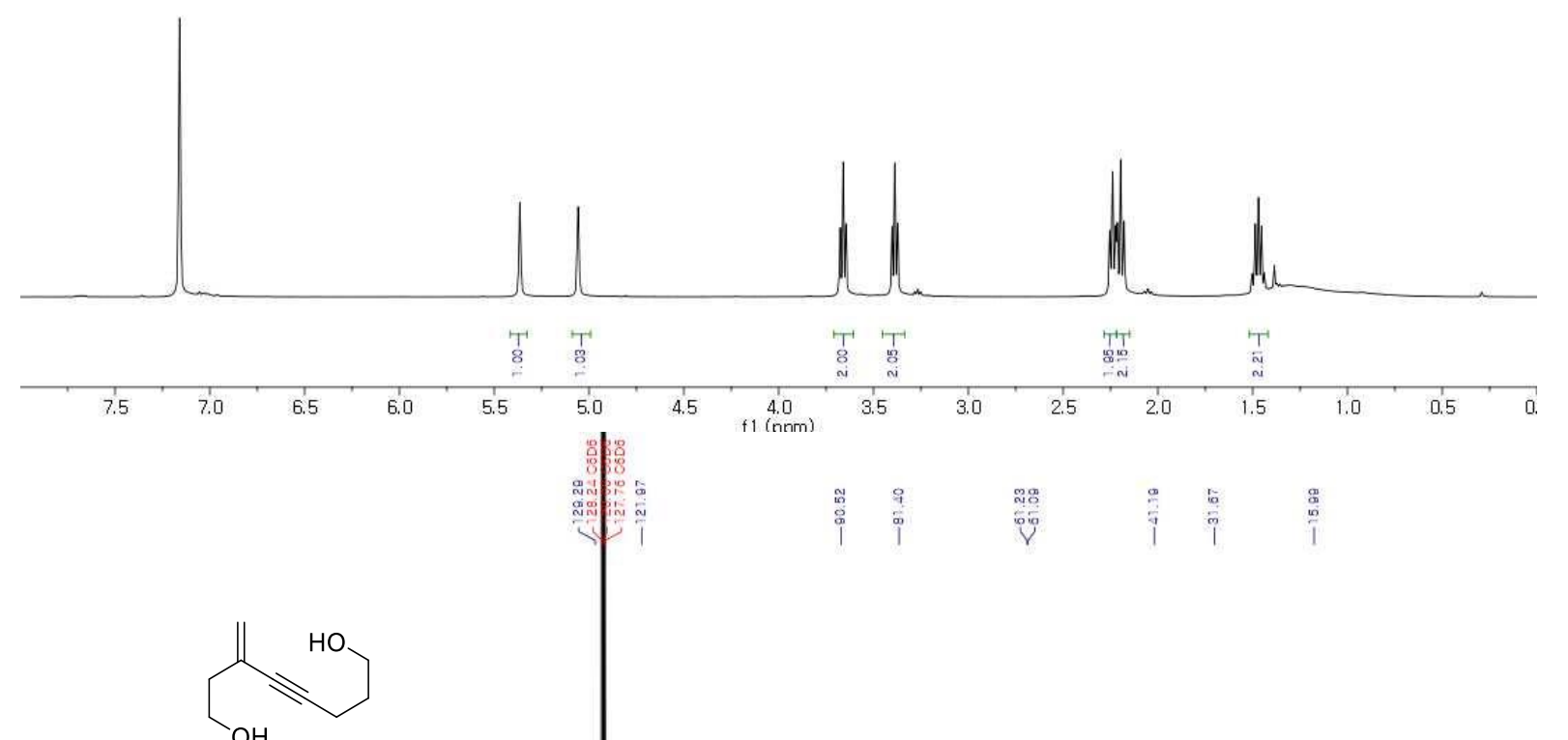

28b

$\left(100 \mathrm{MHz}, \mathrm{C}_{6} \mathrm{D}_{6}\right)$

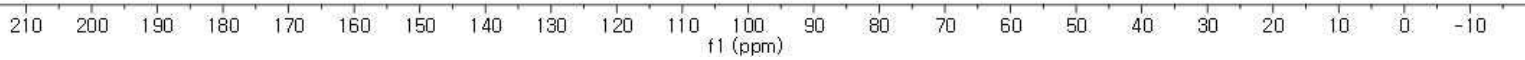




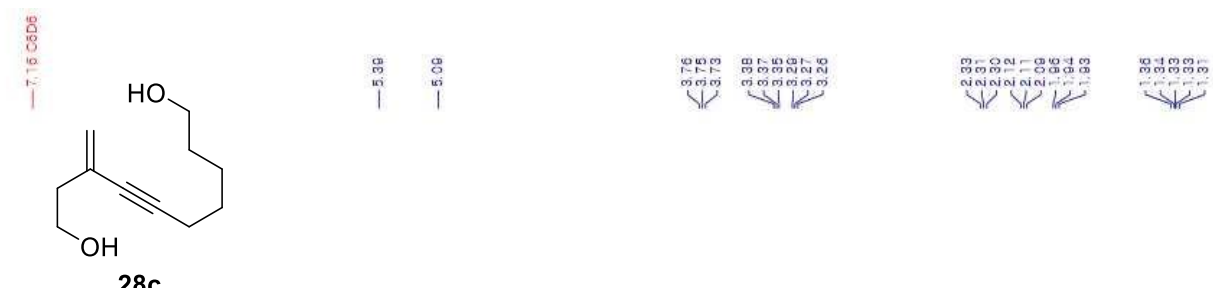

(400MHz, $\mathrm{C}_{6} \mathrm{D}_{6}$ )
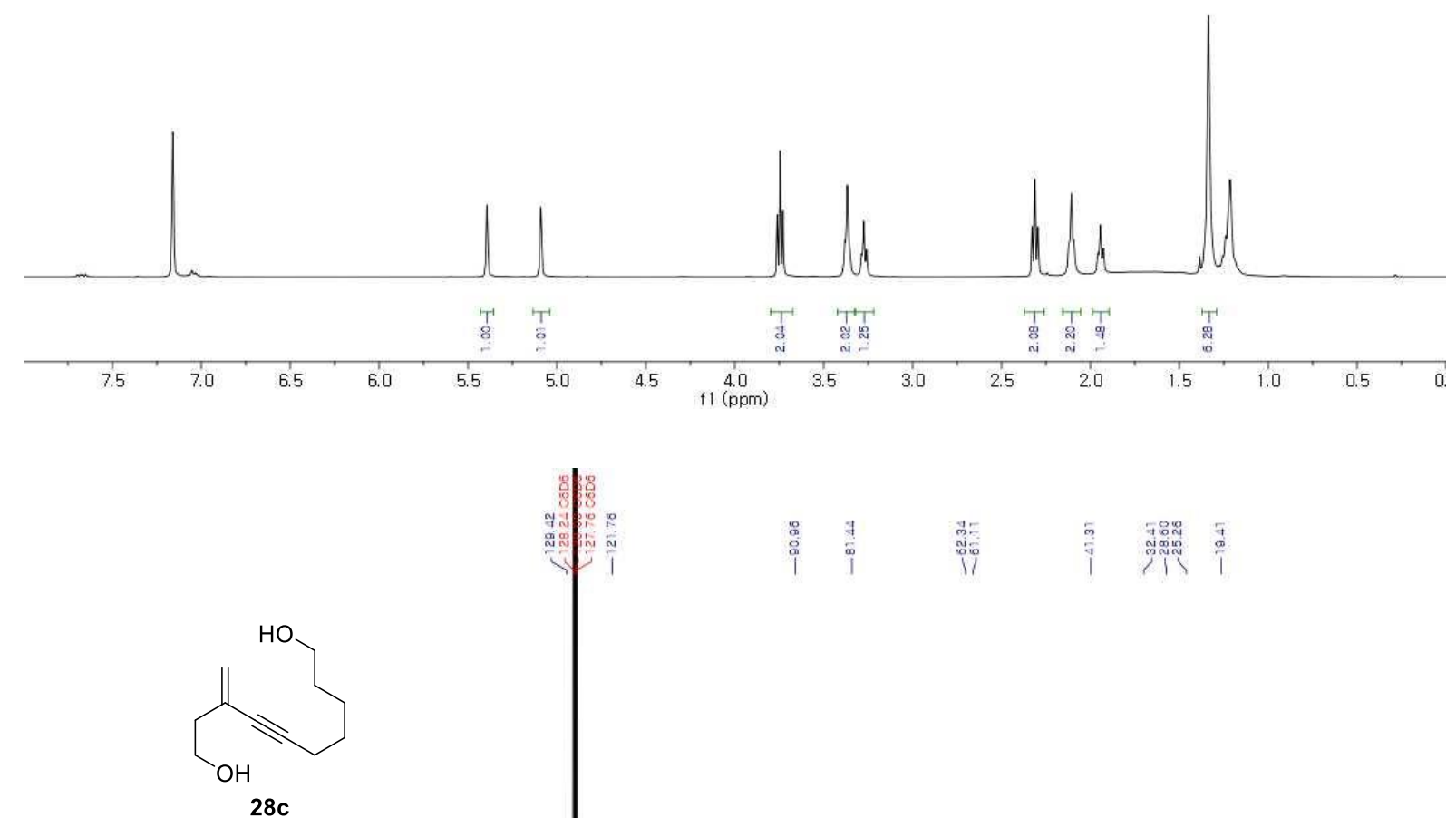

$\left(100 \mathrm{MHz}, \mathrm{C}_{6} \mathrm{D}_{6}\right)$

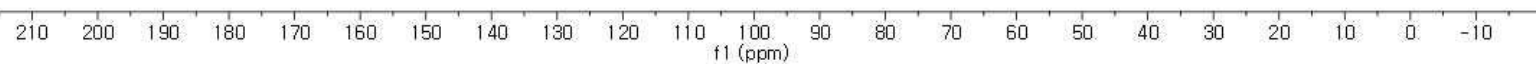



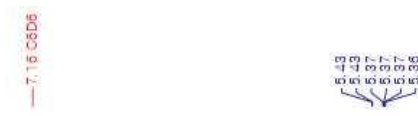

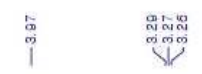

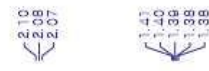<smiles>C=C(C#CCCCO)CO</smiles>

28d

(400MHz, $\mathrm{C}_{6} \mathrm{D}_{6}$ )
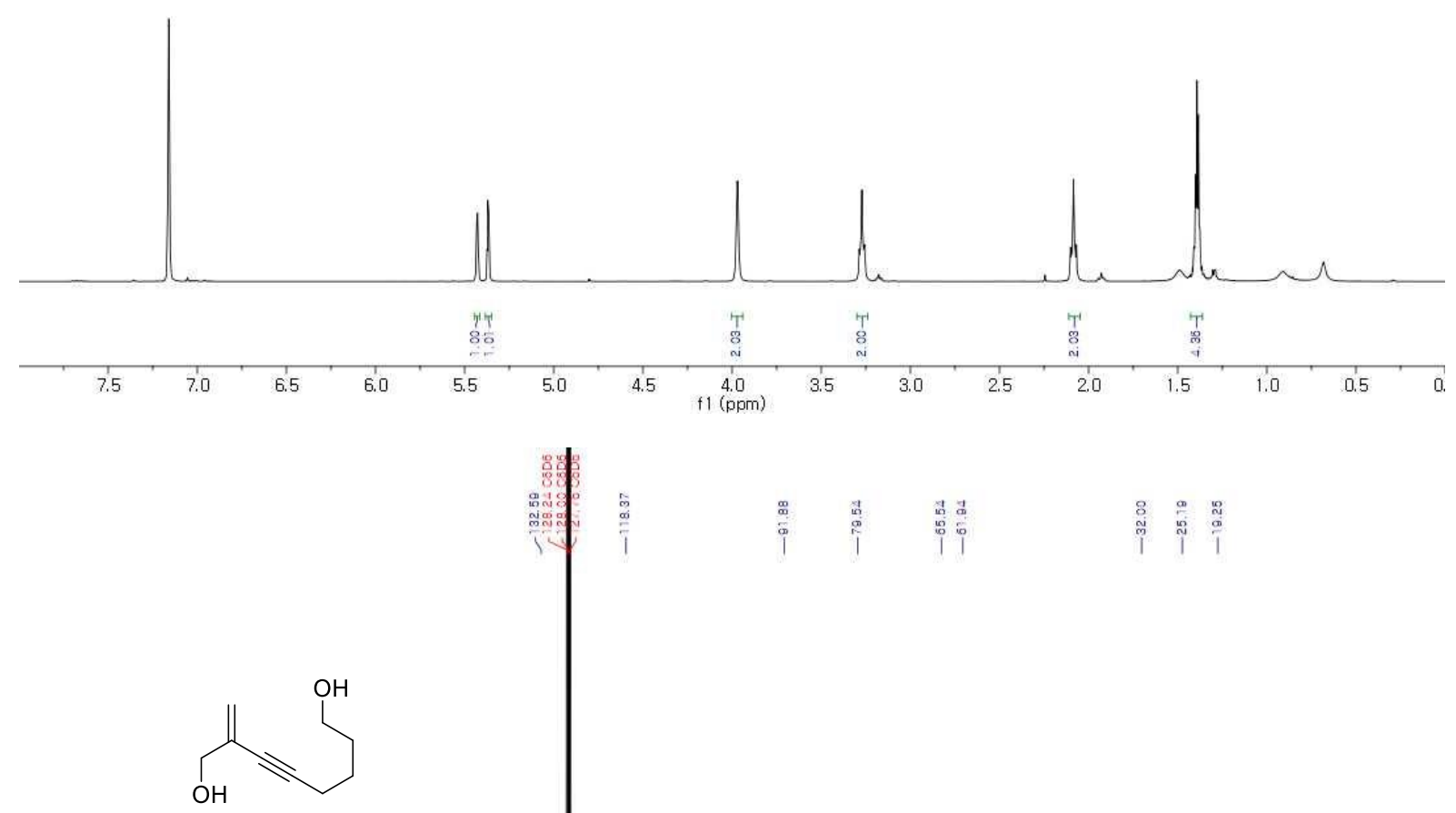

28d

$\left(100 \mathrm{MHz}, \mathrm{C}_{6} \mathrm{D}_{6}\right)$

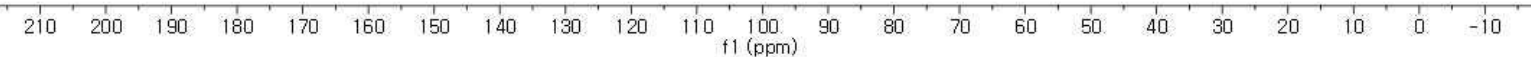




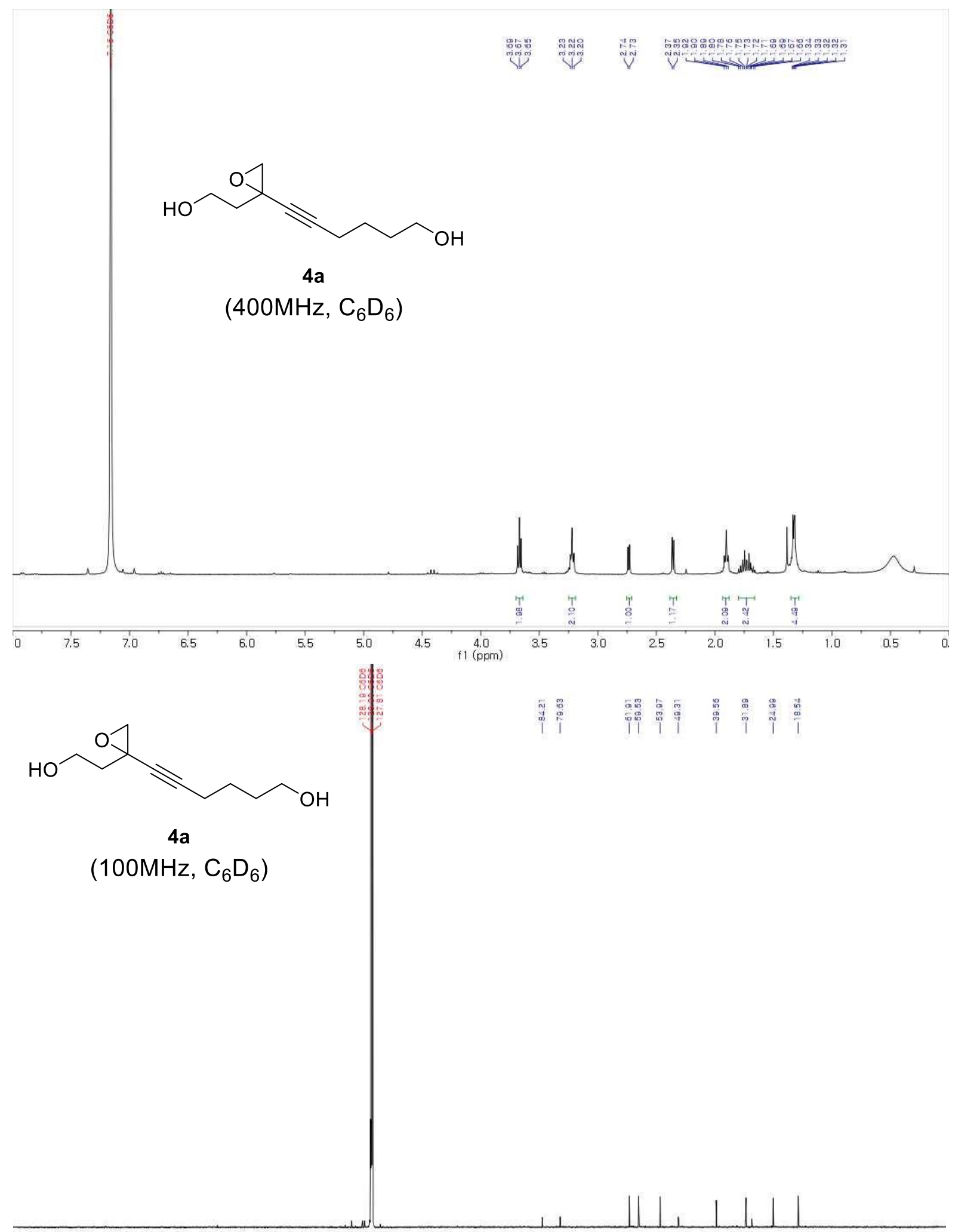

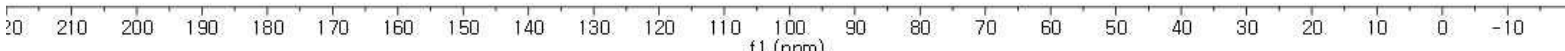




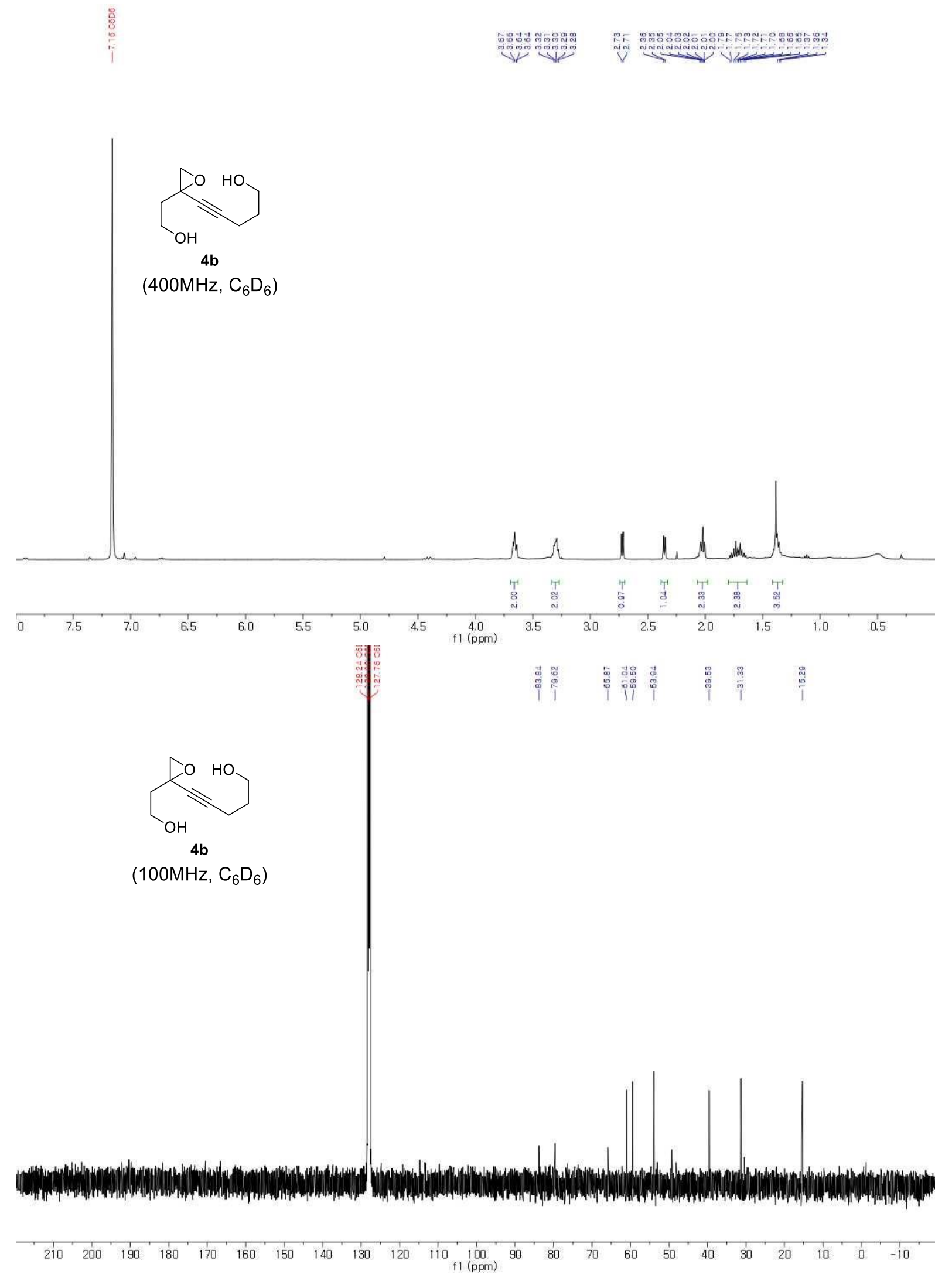




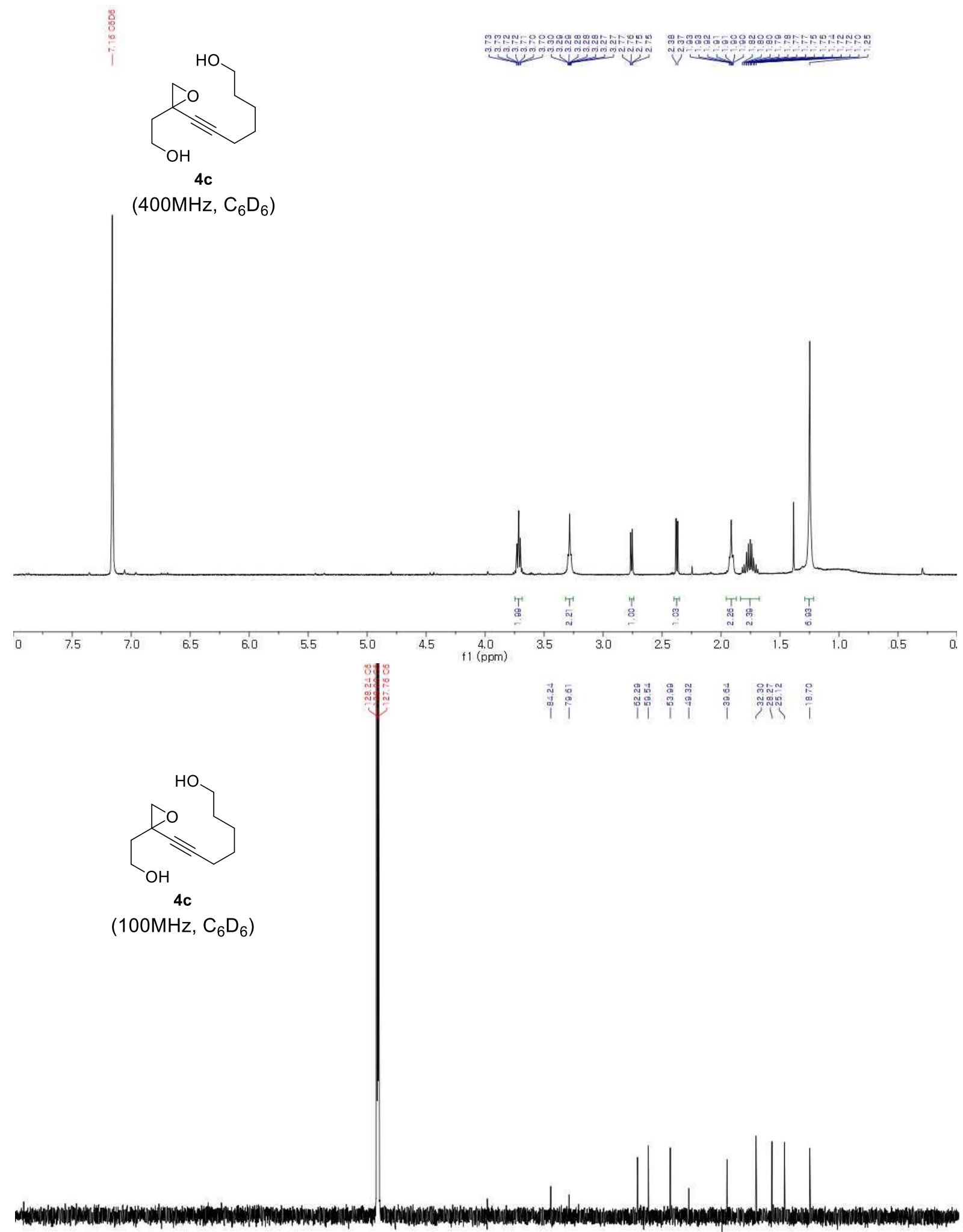

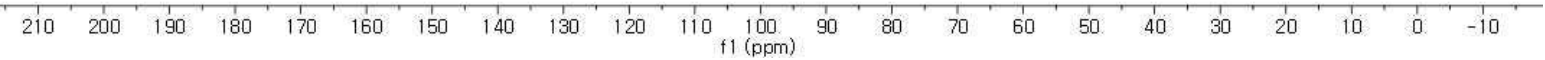




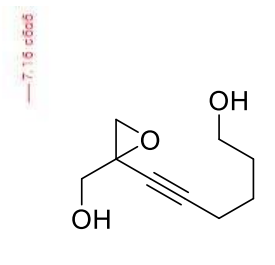

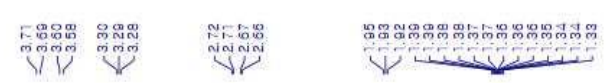

$\left(400 \mathrm{MHz}, \mathrm{C}_{6} \mathrm{D}_{6}\right)$
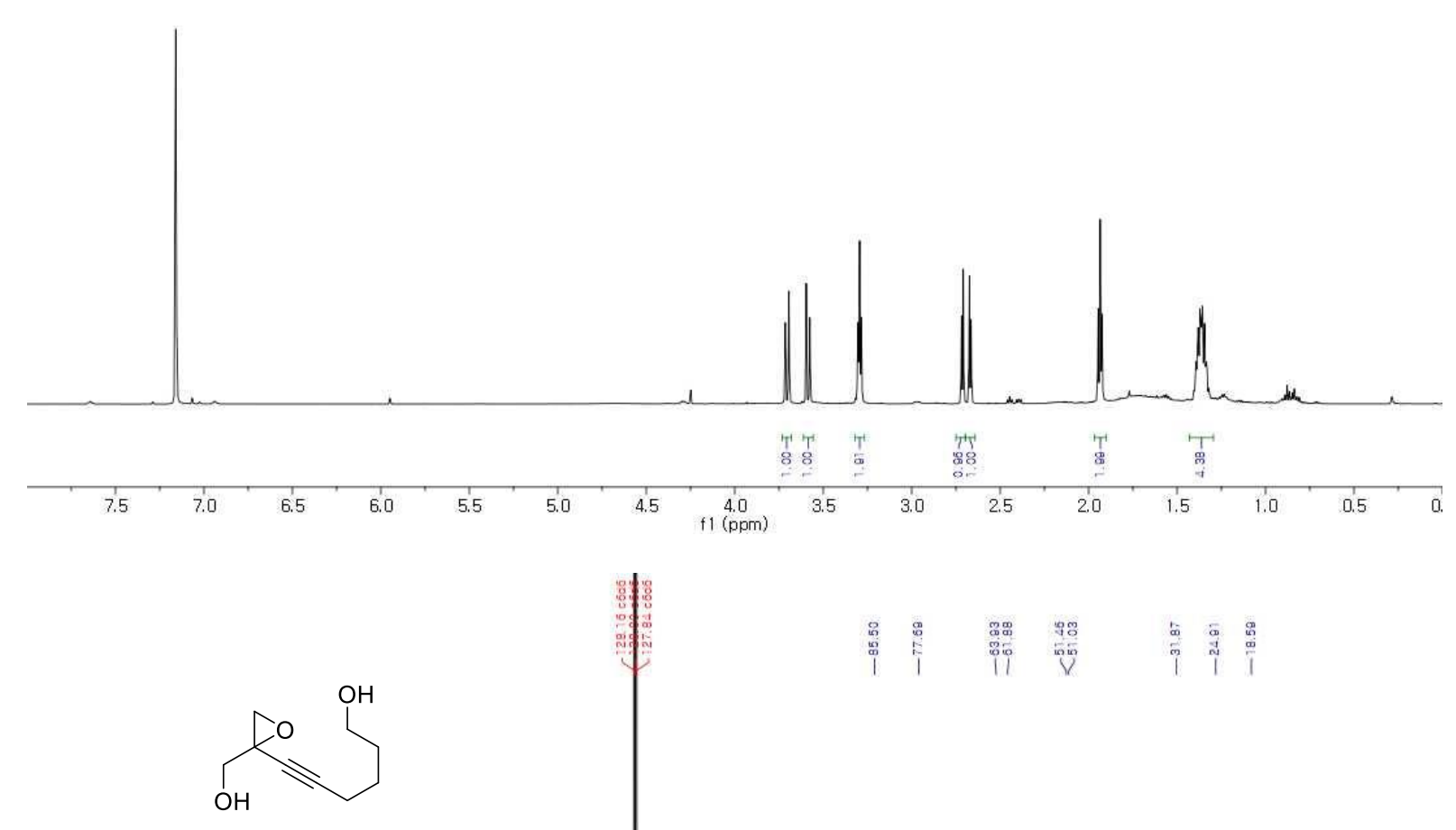

$4 d$

$\left(100 \mathrm{MHz}, \mathrm{C}_{6} \mathrm{D}_{6}\right)$ 


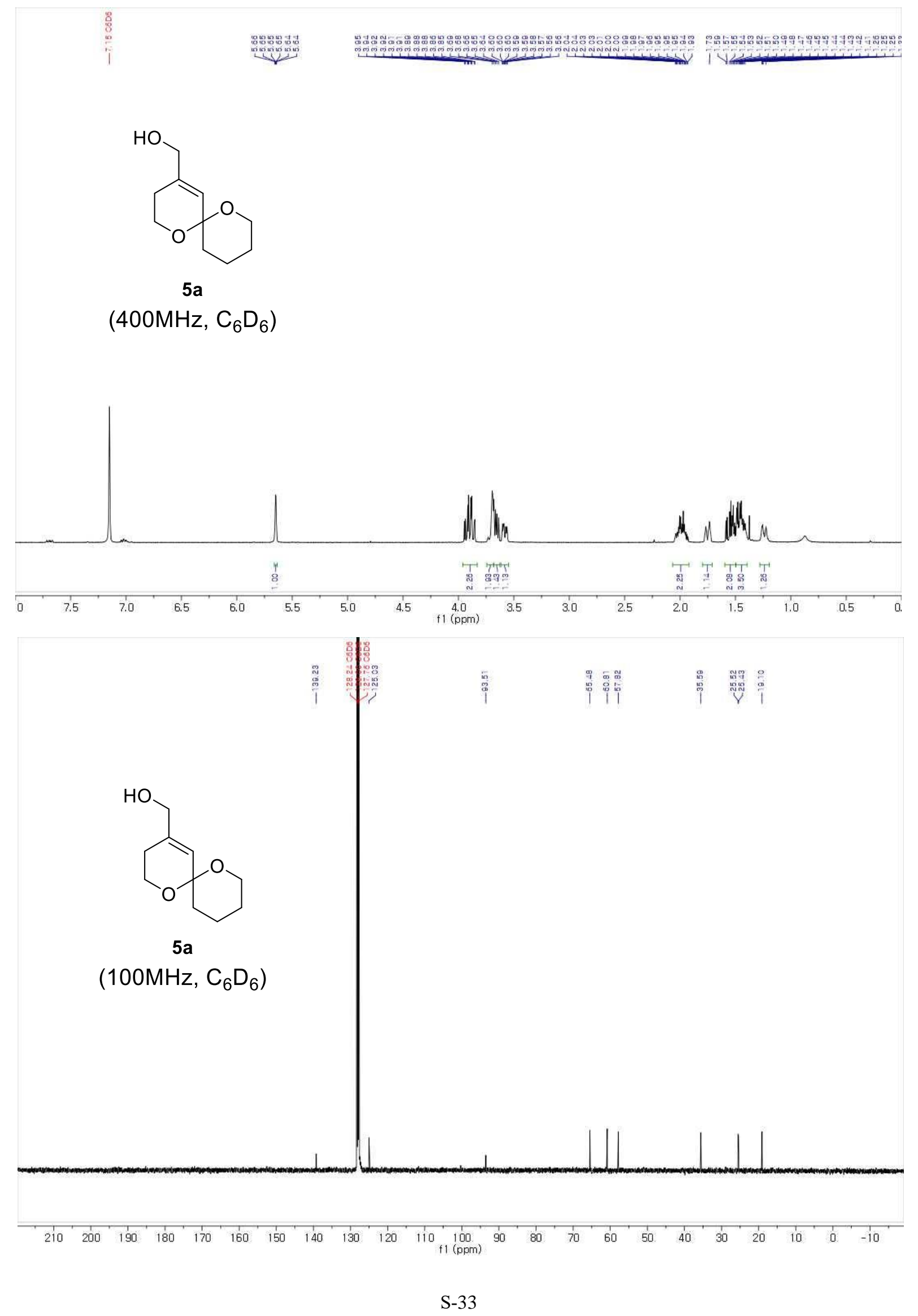



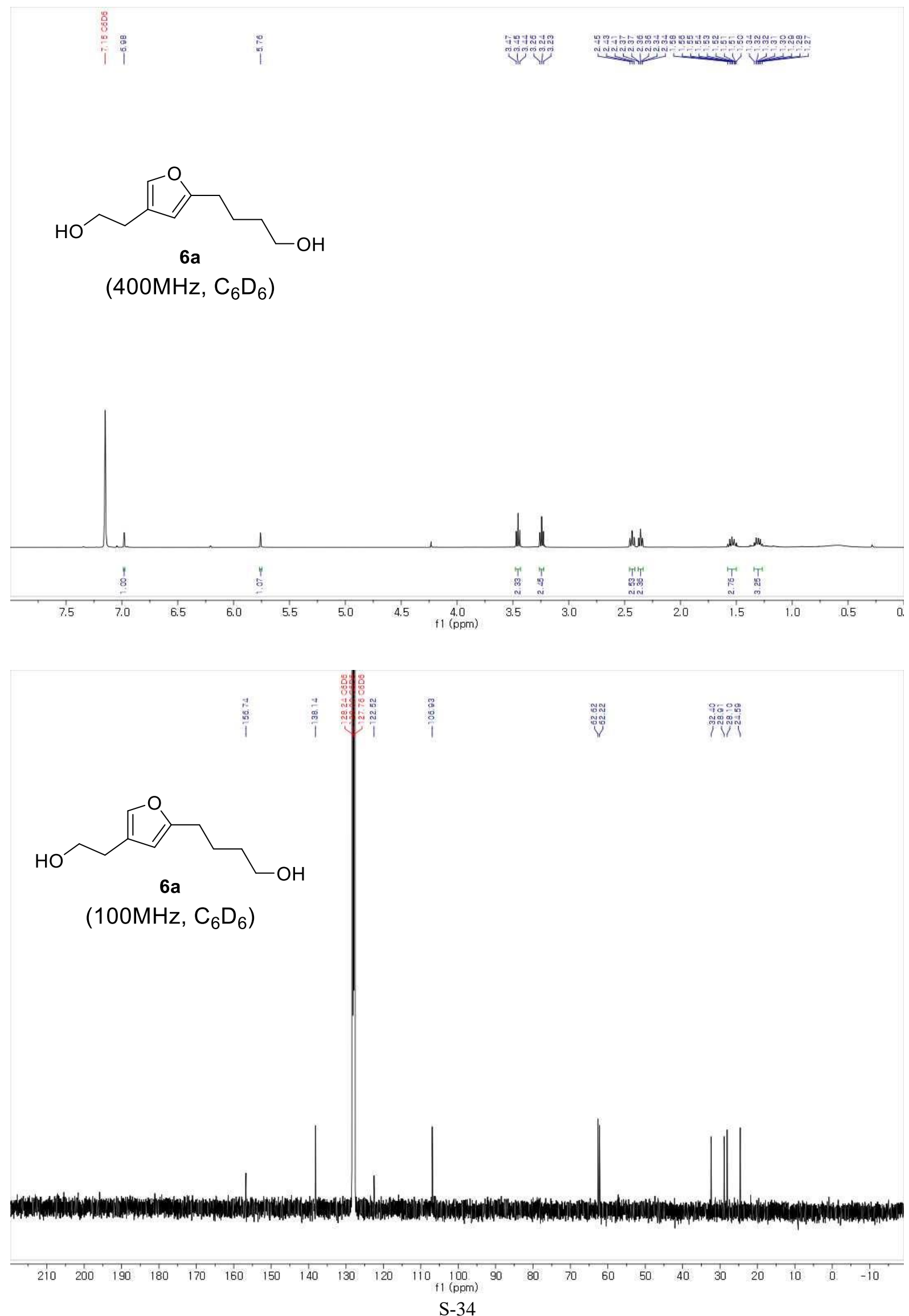

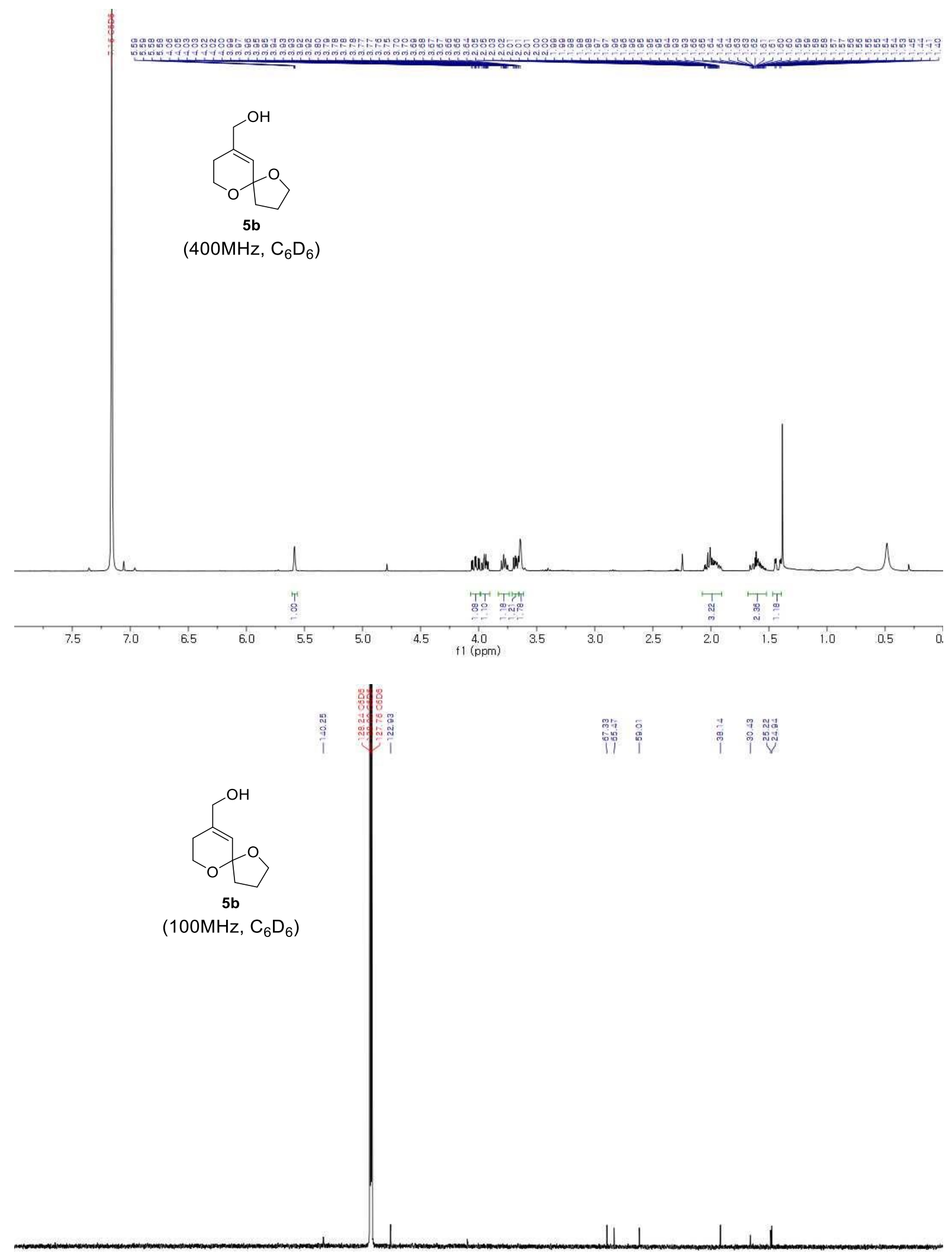

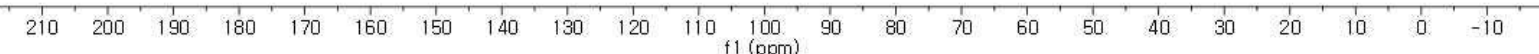
S-35 

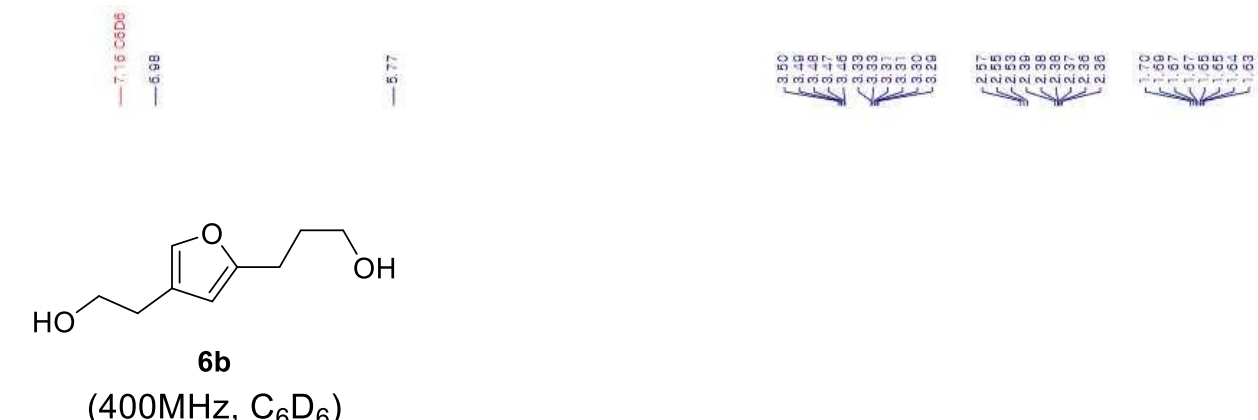

$\left(400 \mathrm{MHz}, \mathrm{C}_{6} \mathrm{D}_{6}\right)$
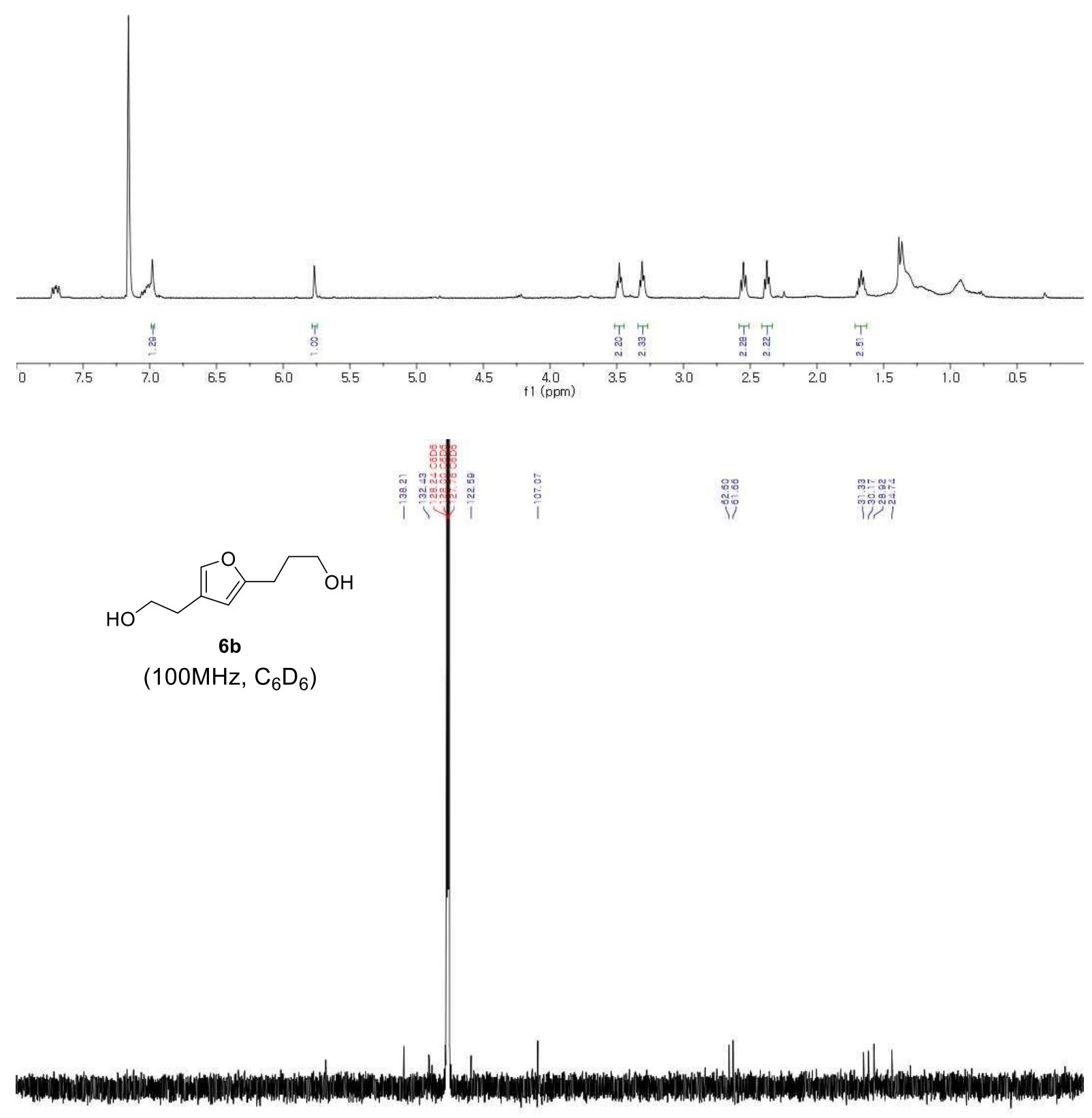

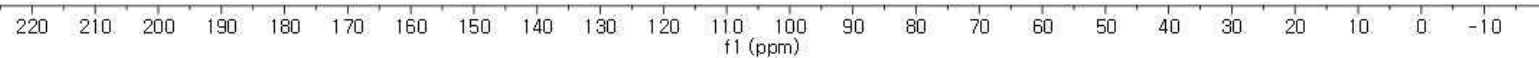
S-36 


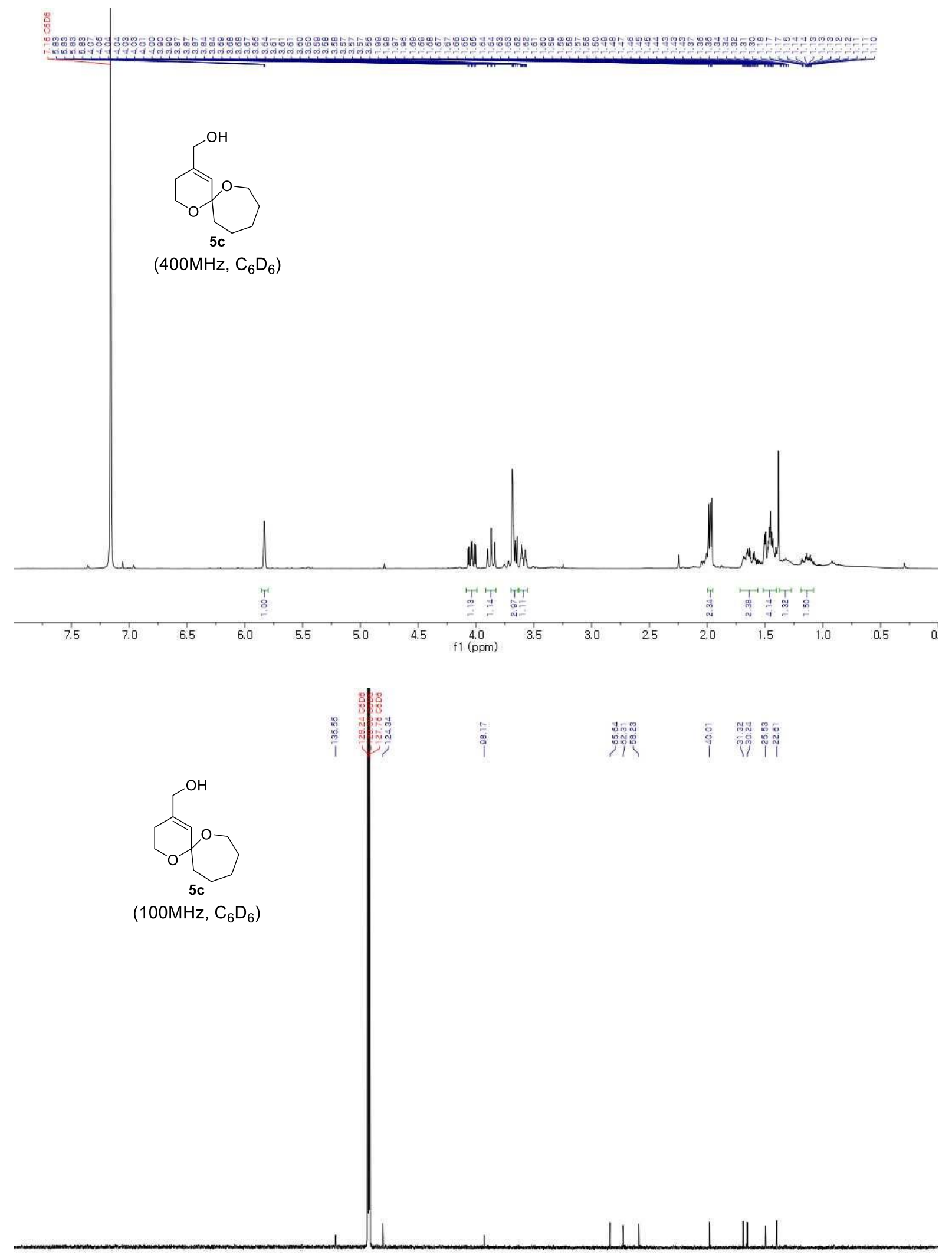

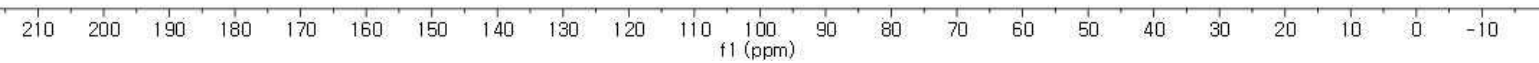
S-37 


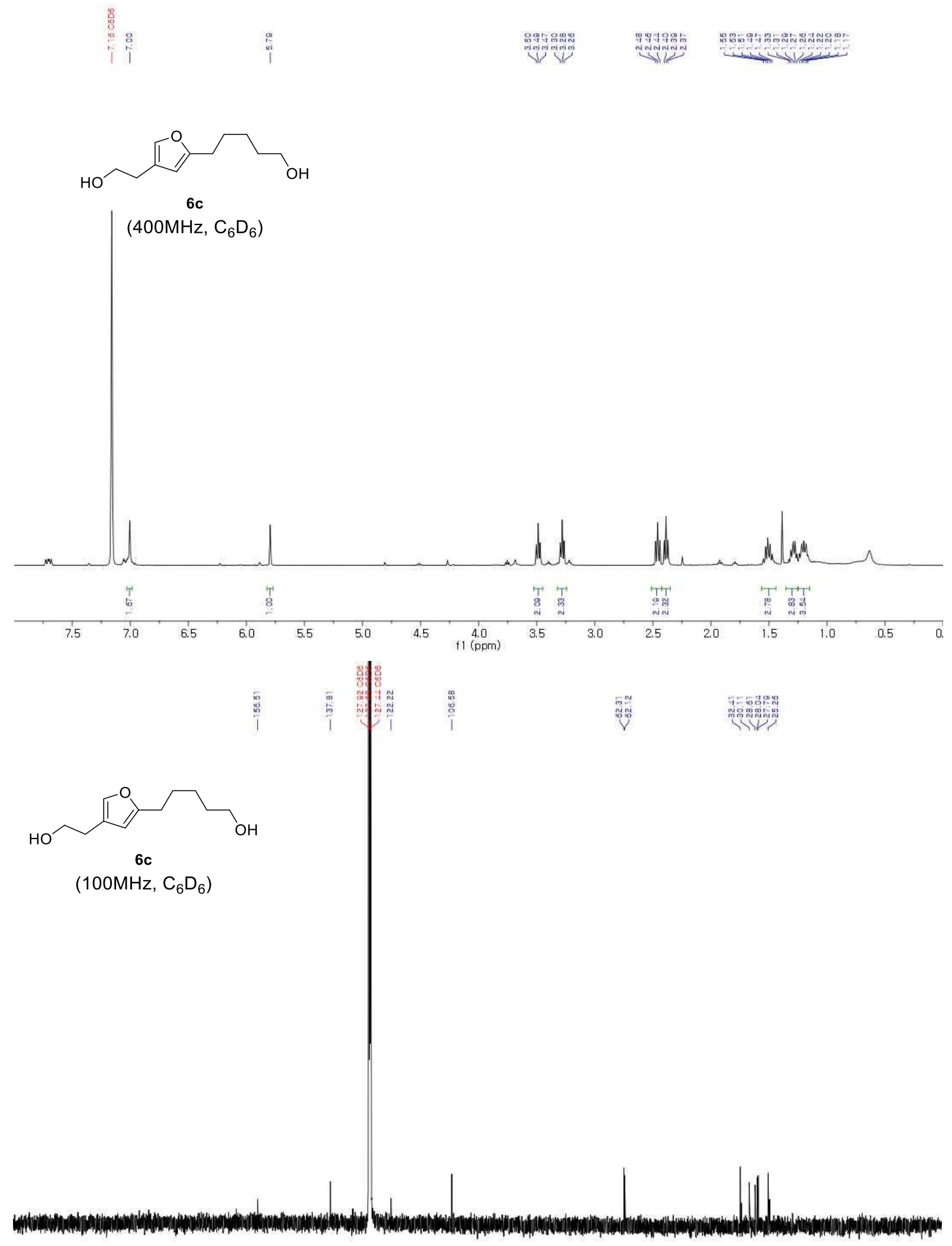

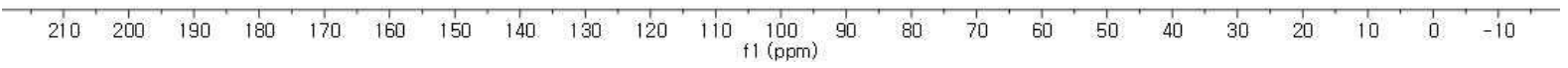




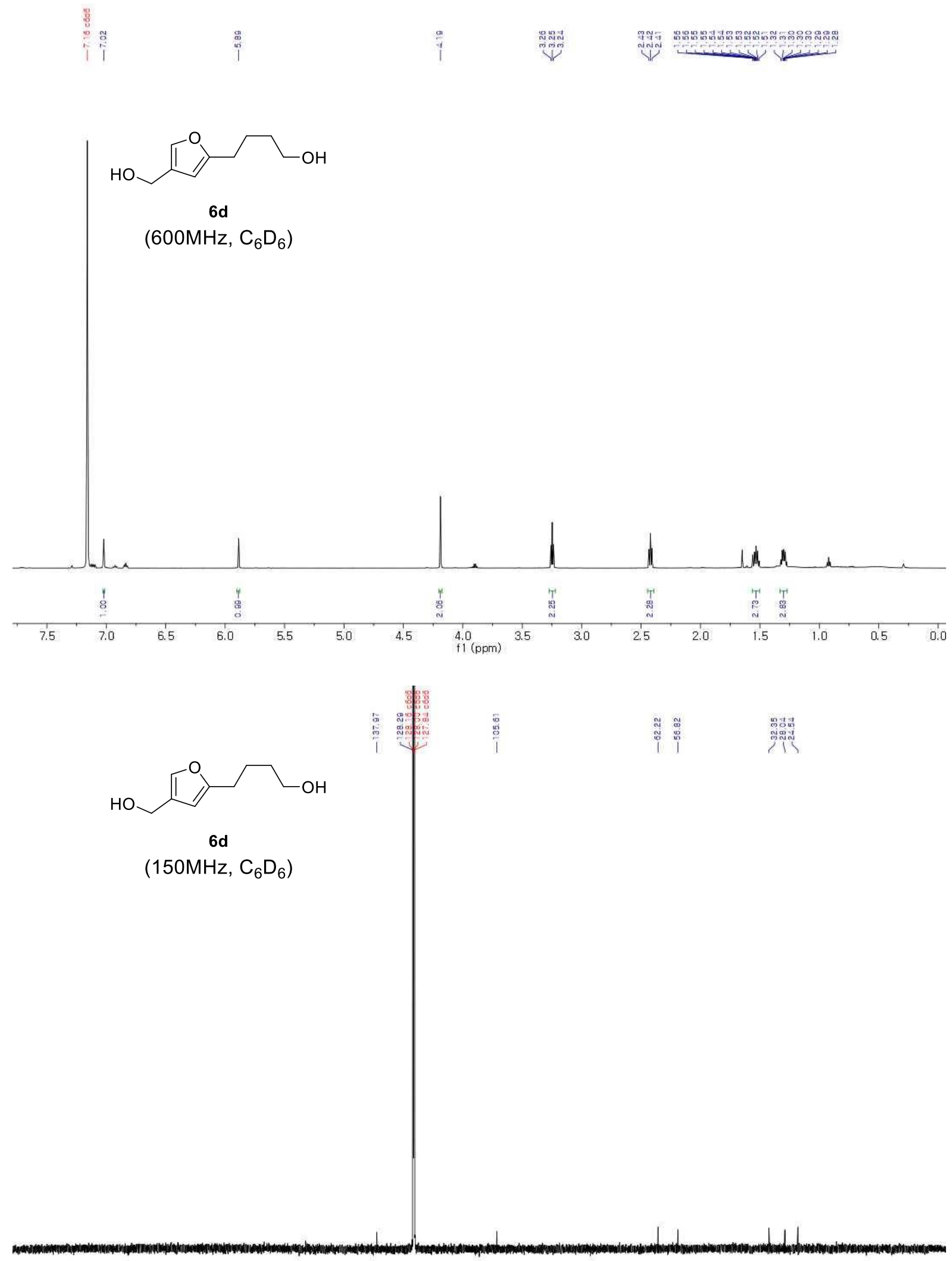

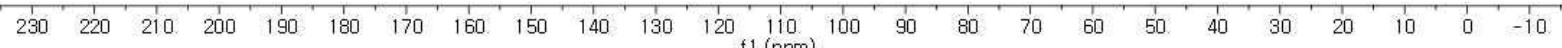
S-39 

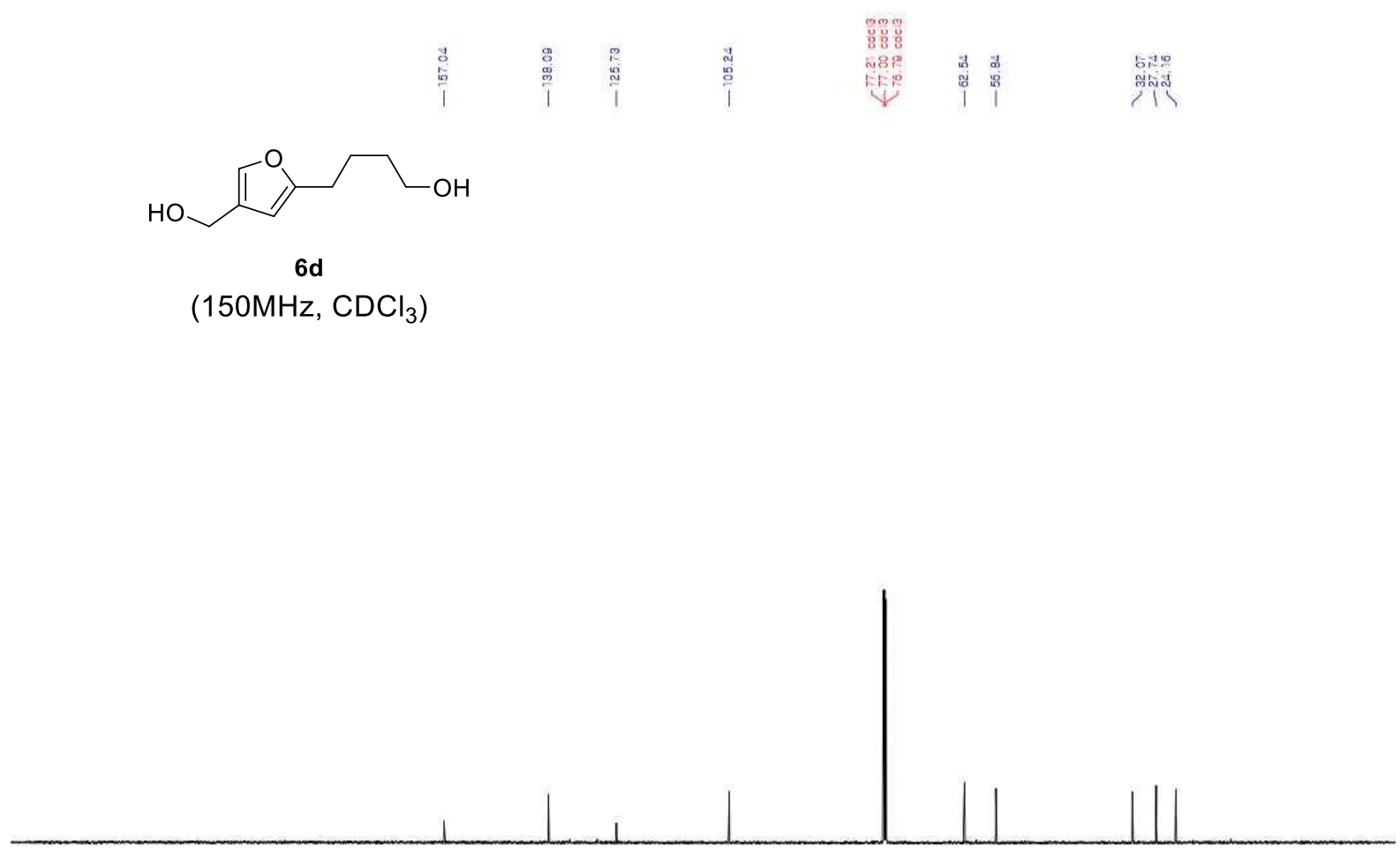

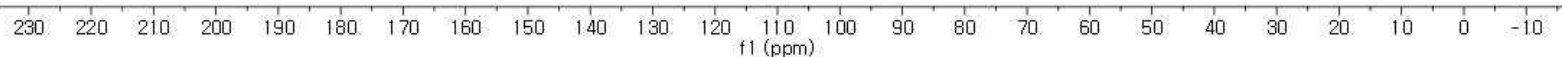




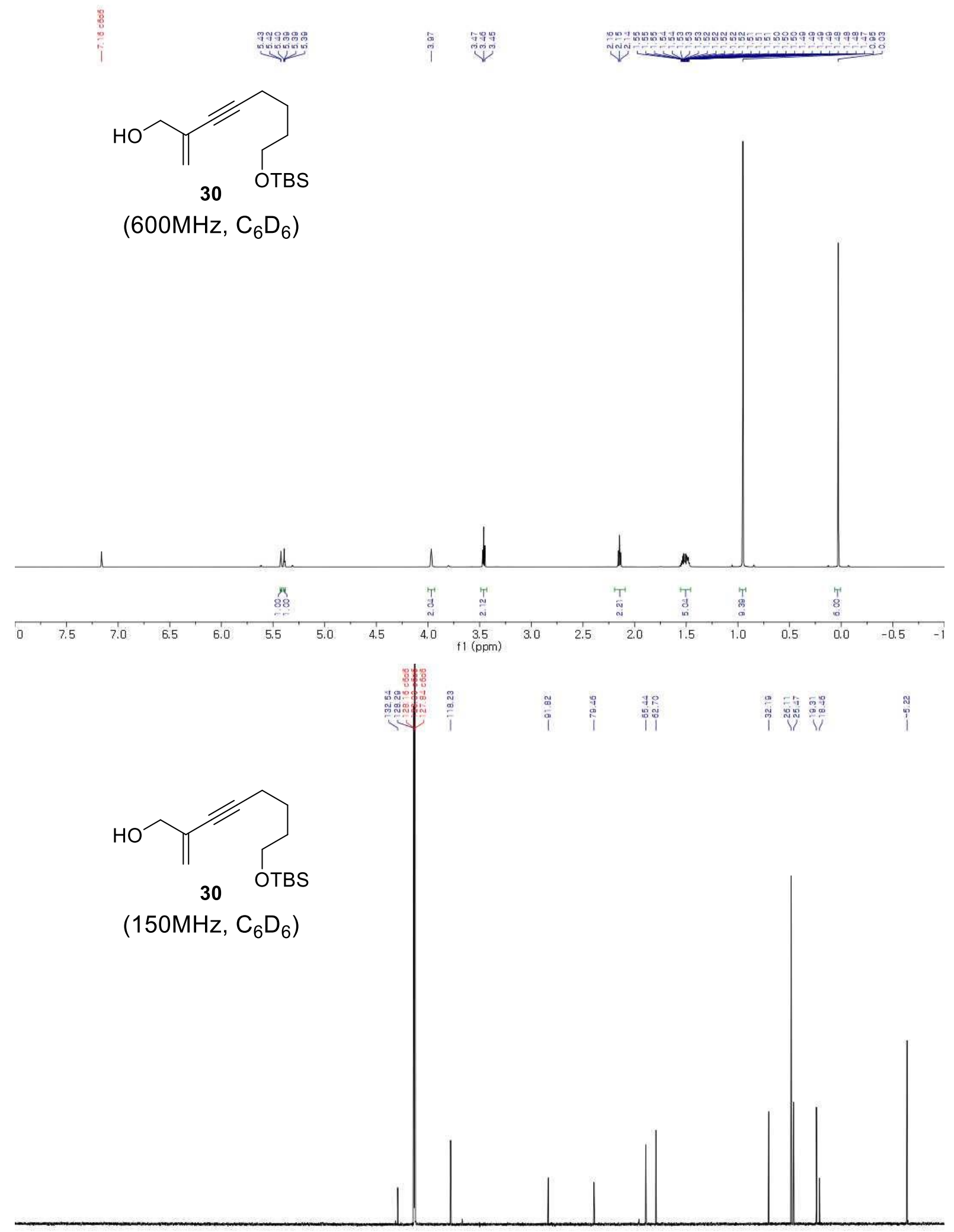

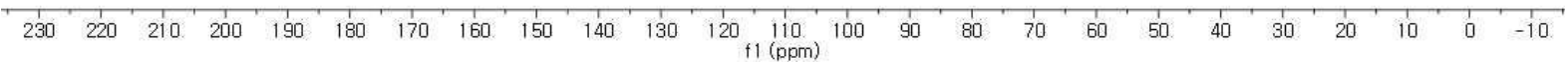
S-41 


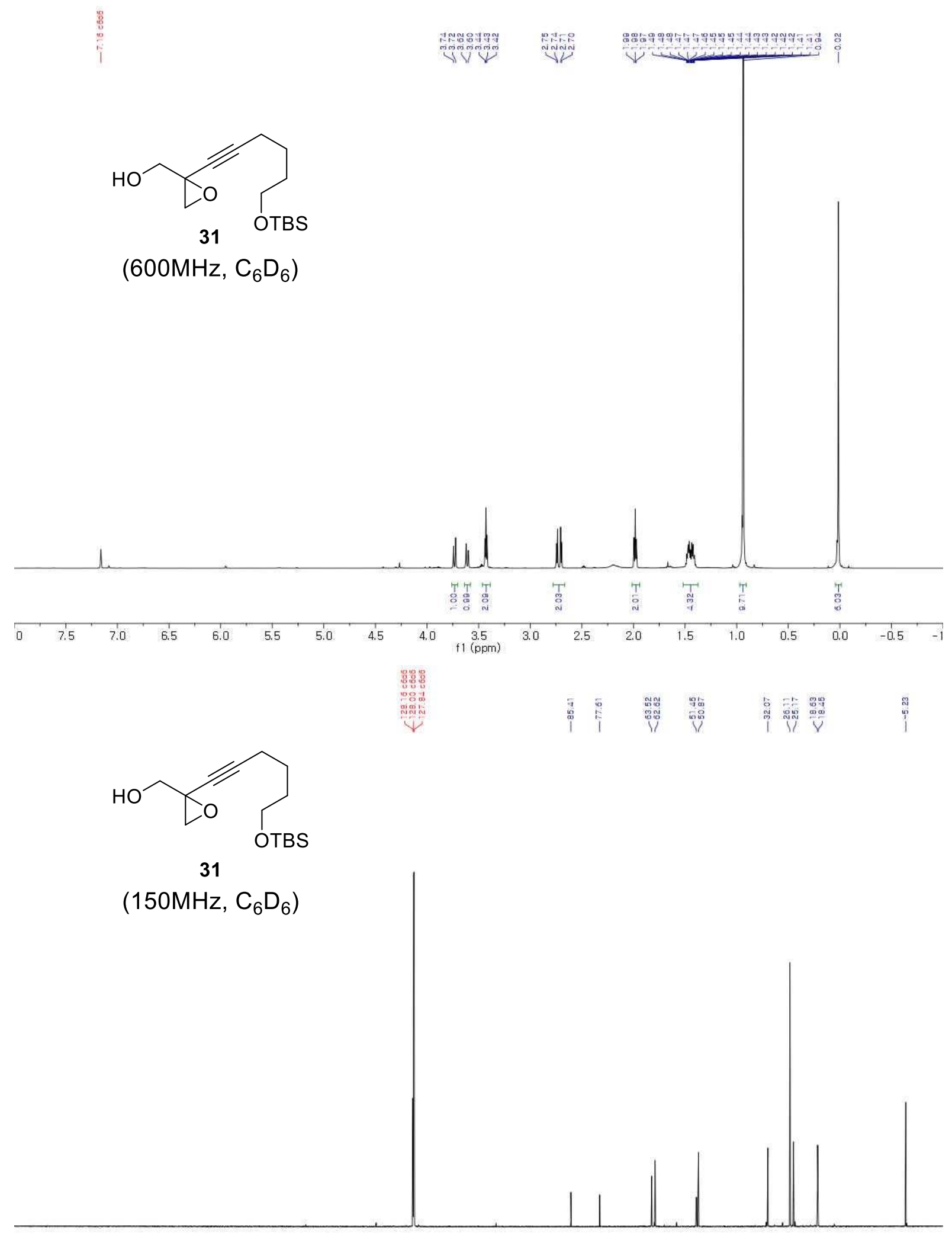

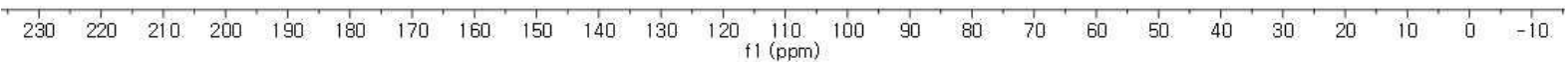
S-42 

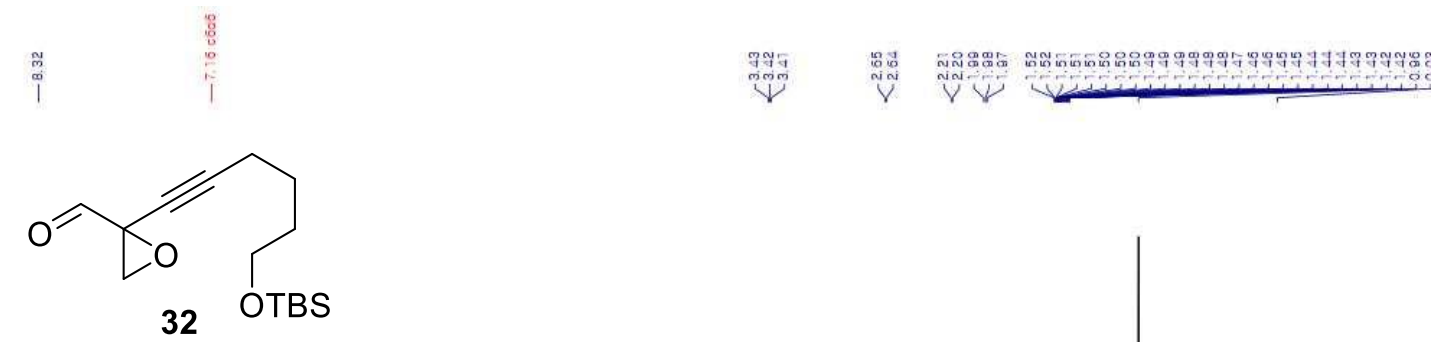

(600MHz, $\mathrm{C}_{6} \mathrm{D}_{6}$ )
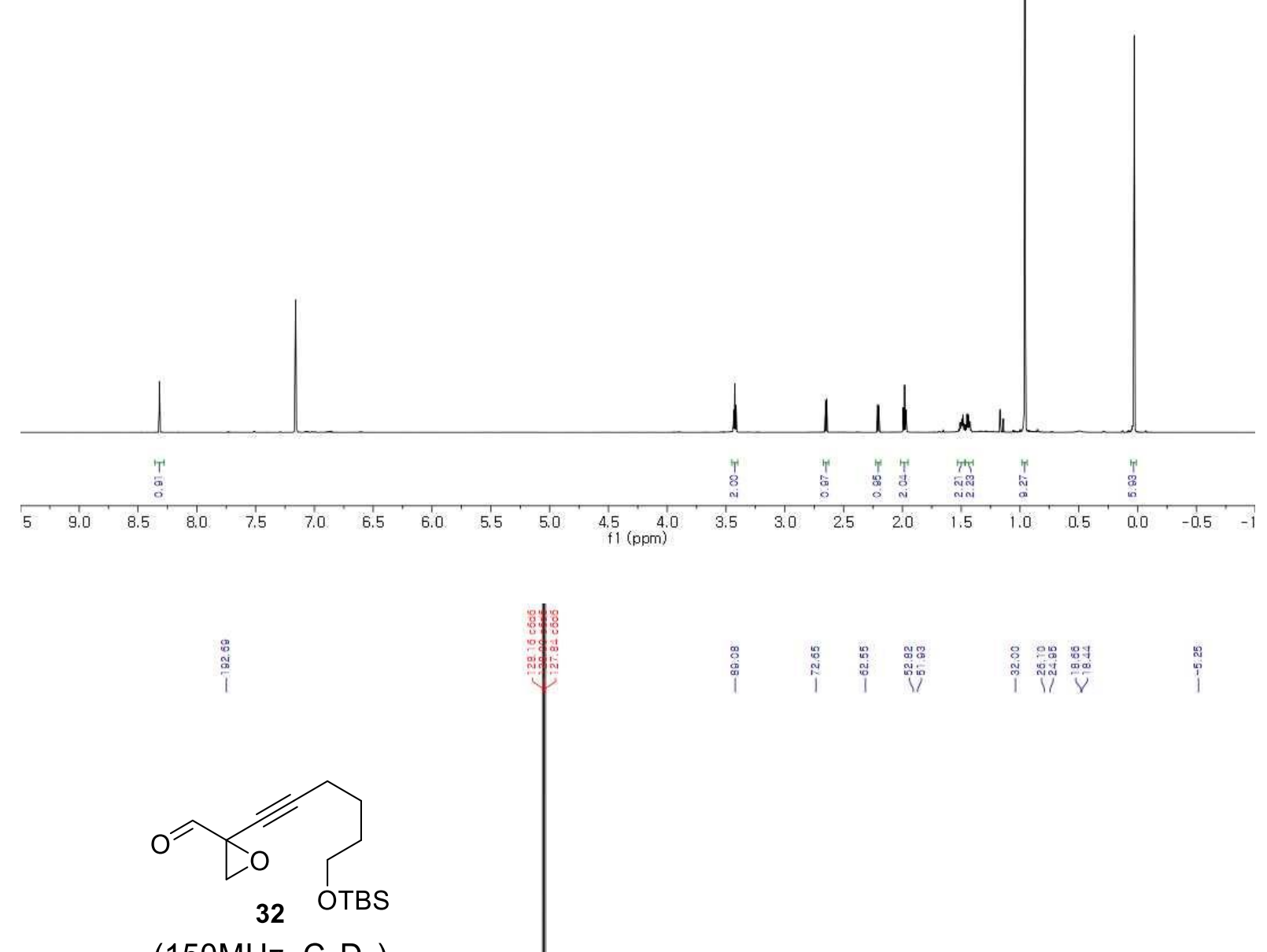

$\left(150 \mathrm{MHz}, \mathrm{C}_{6} \mathrm{D}_{6}\right.$ )

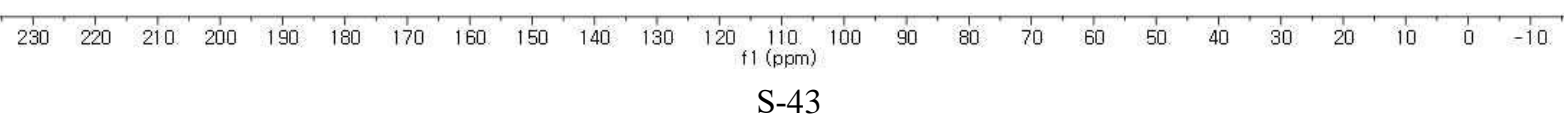




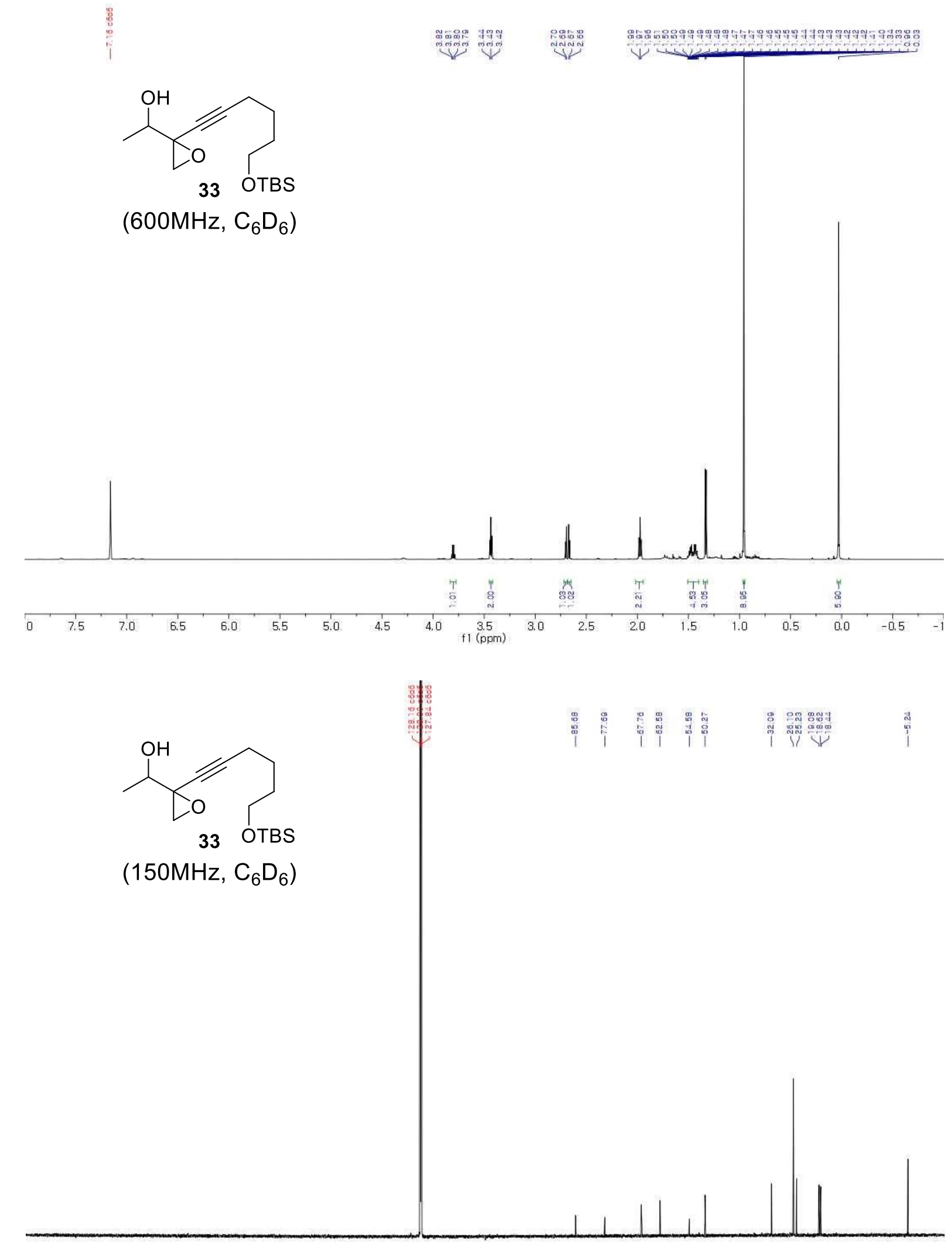

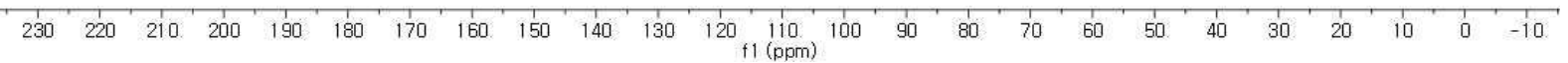
S-44 

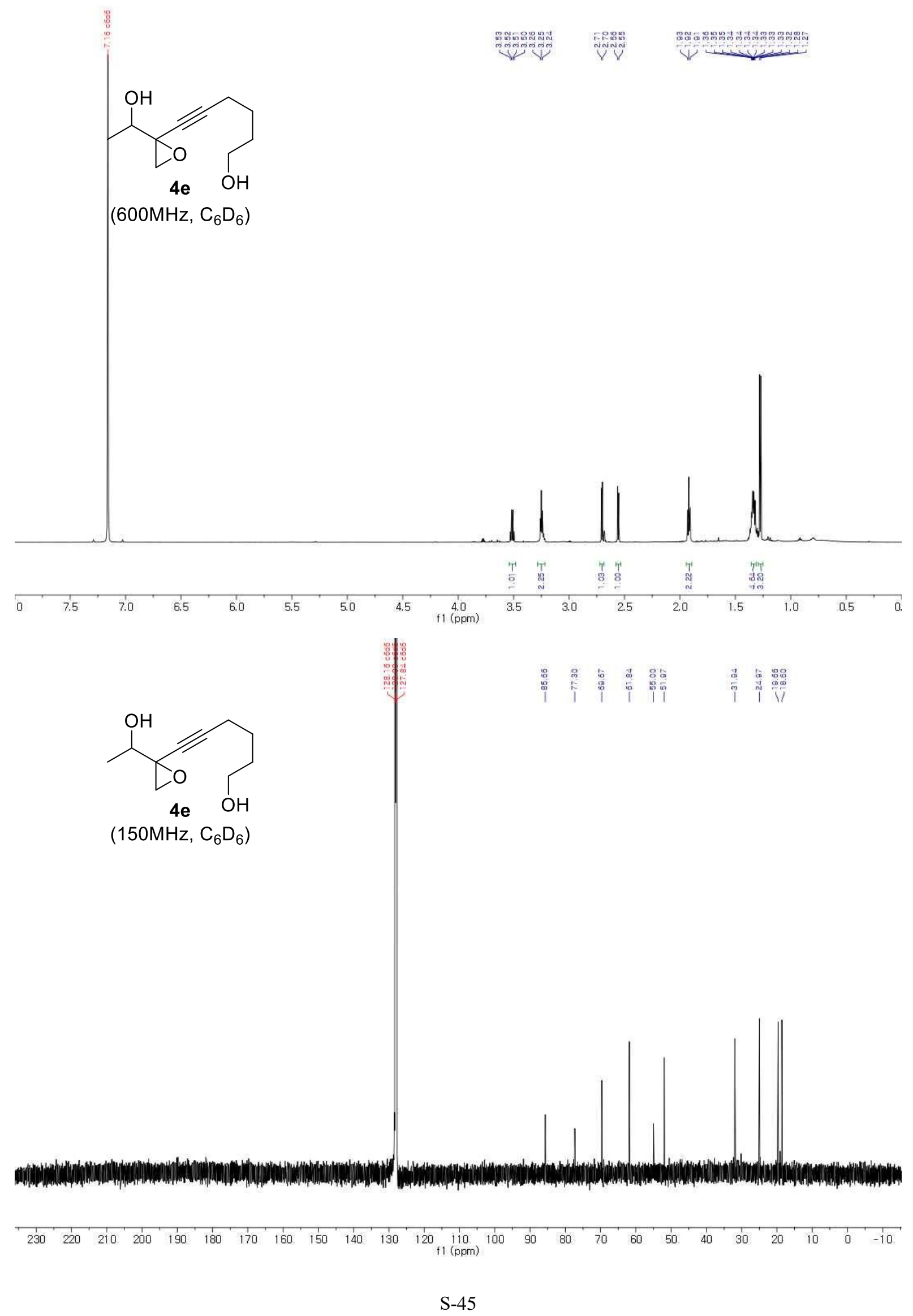

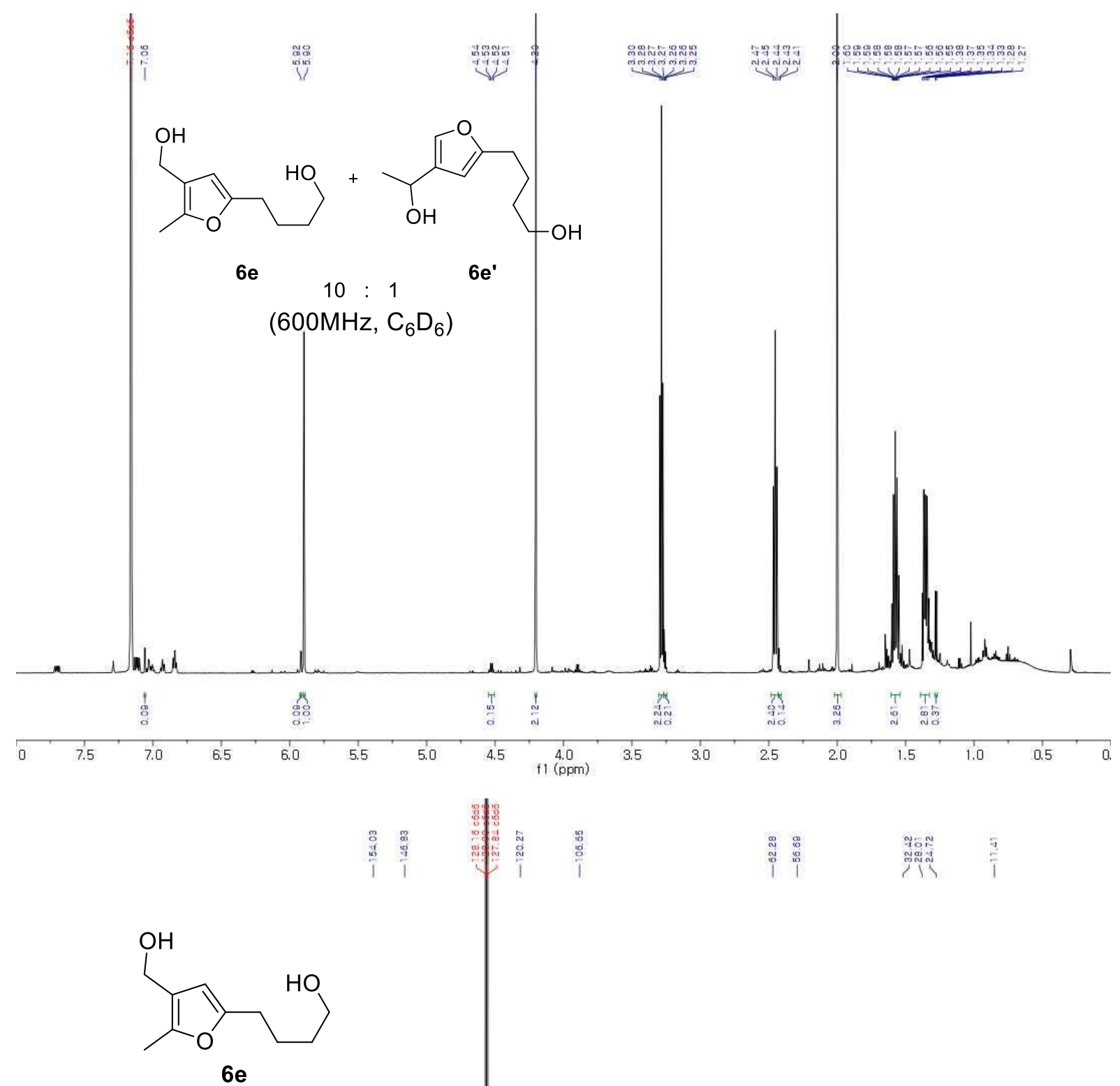

$\left(150 \mathrm{MHz}, \mathrm{C}_{6} \mathrm{D}_{6}\right)$

$\begin{array}{lllllllllllllllllllllllllllllllllllllll}230 & 220 & 210 & 200 & 190 & 180 & 170 & 160 & 150 & 140 & 130 & 120 & 110 & 100 & 90 & 80 & 70 & 60 & 50 & 40 & 30 & 20 & 10 & 0 & -10\end{array}$ 


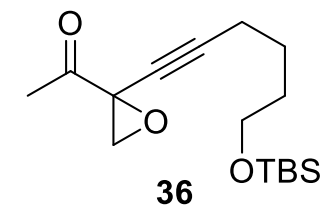

$\left(600 \mathrm{MHz}, \mathrm{C}_{6} \mathrm{D}_{6}\right)$
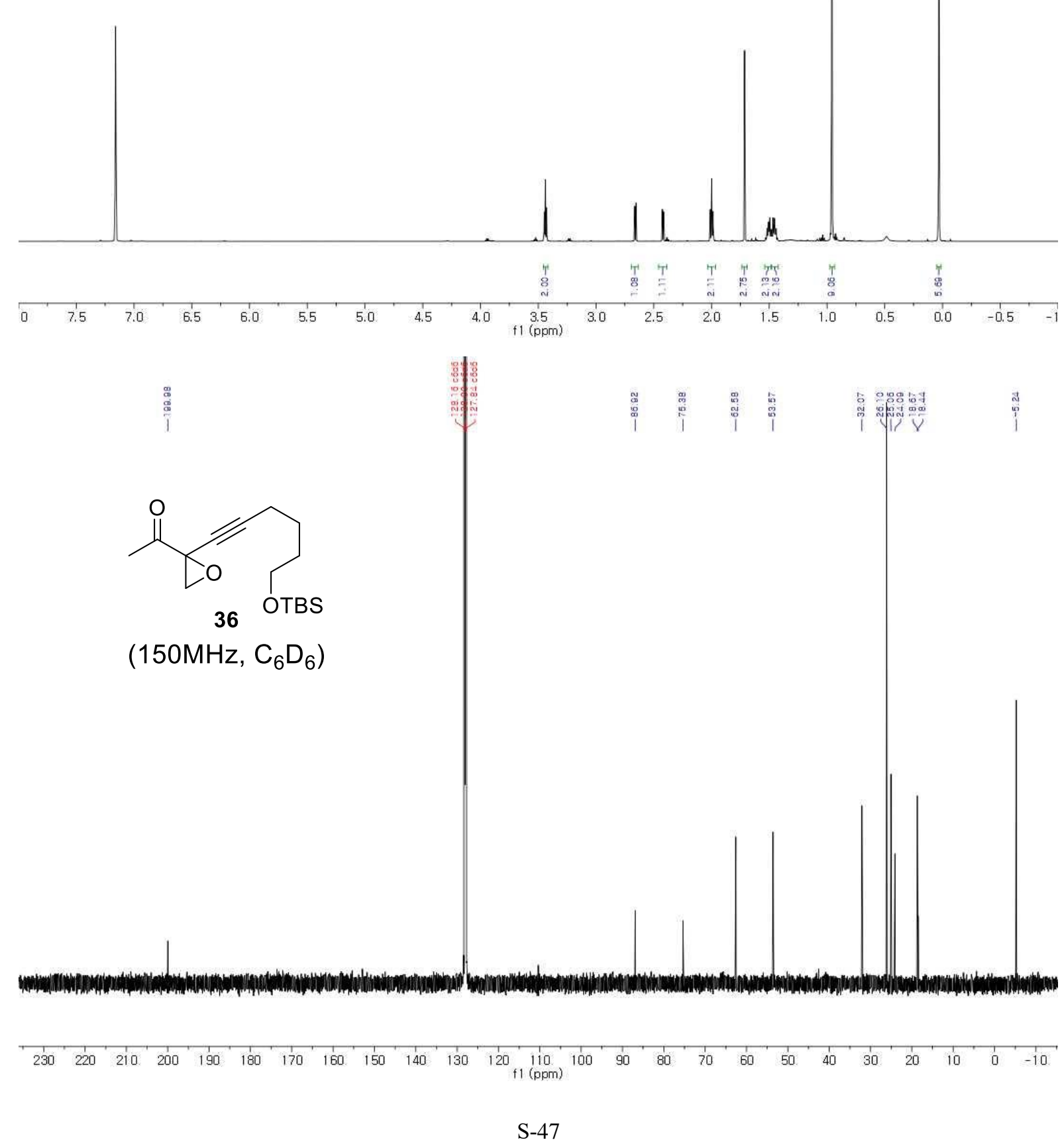


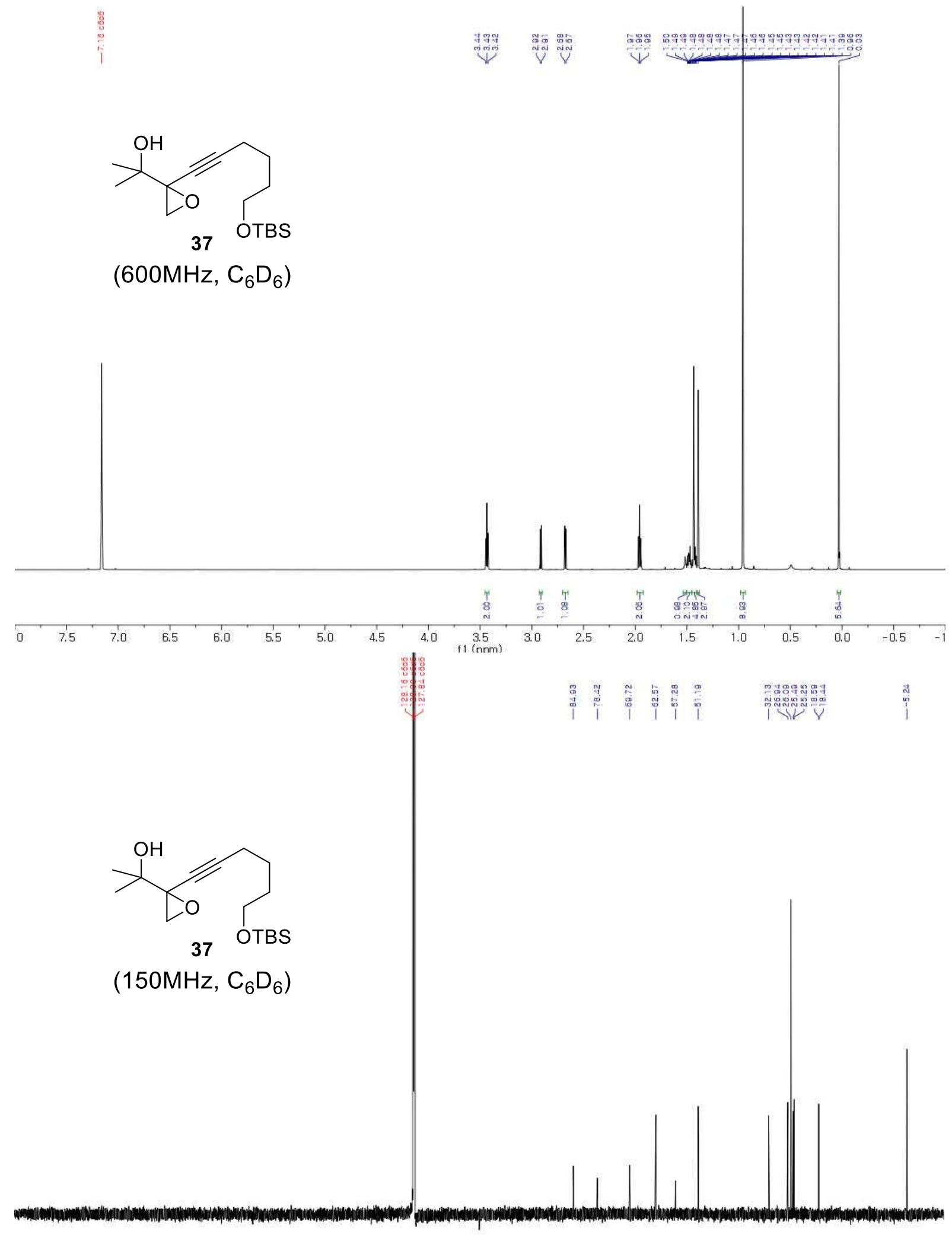

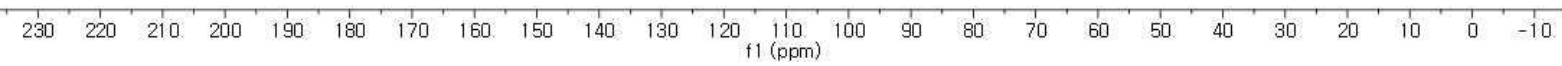




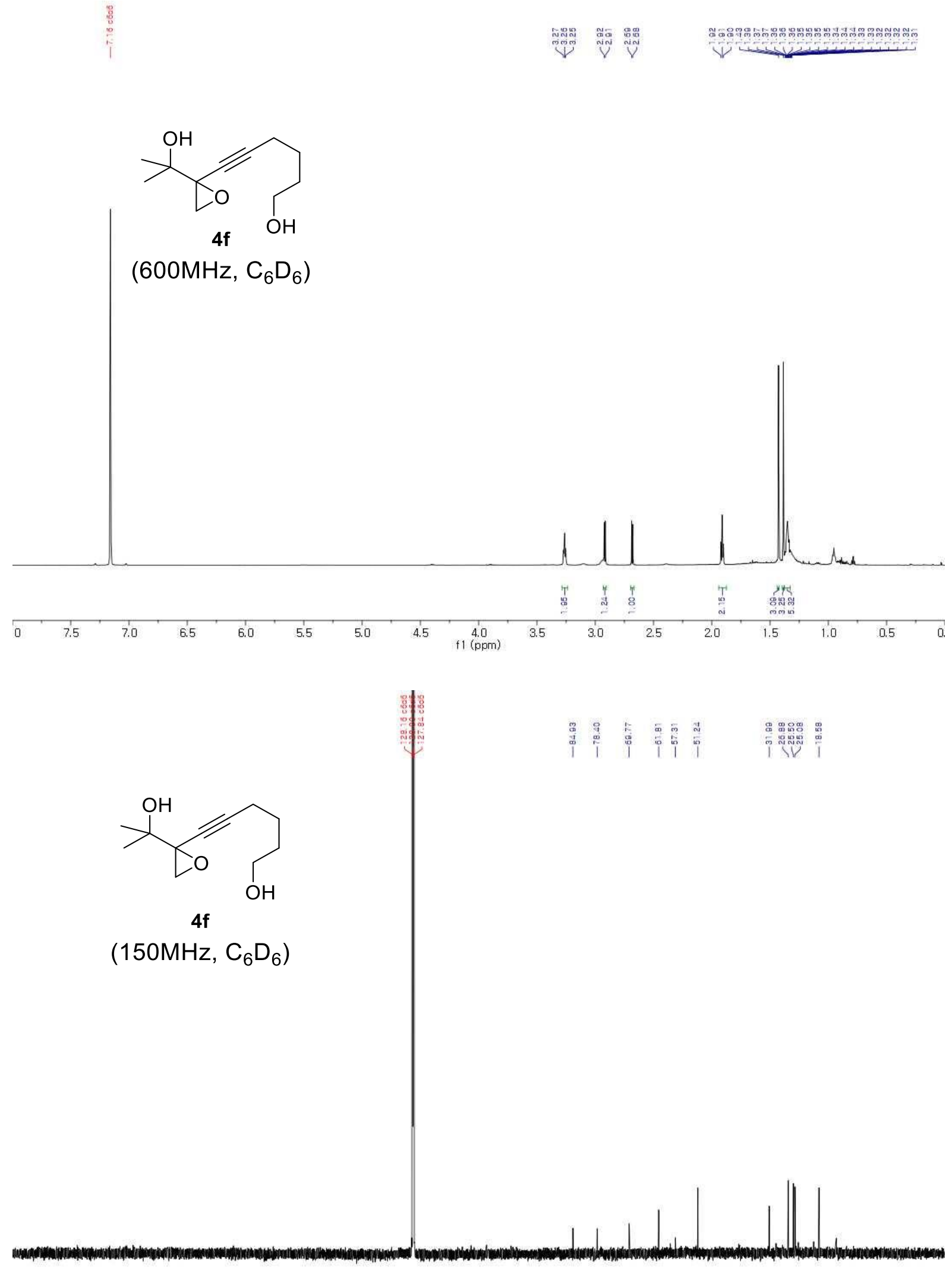

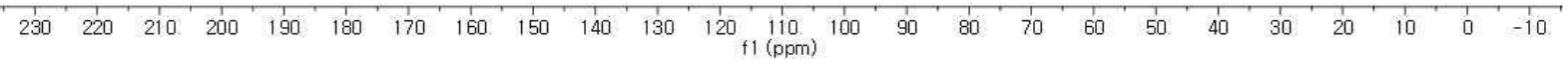
S-49 


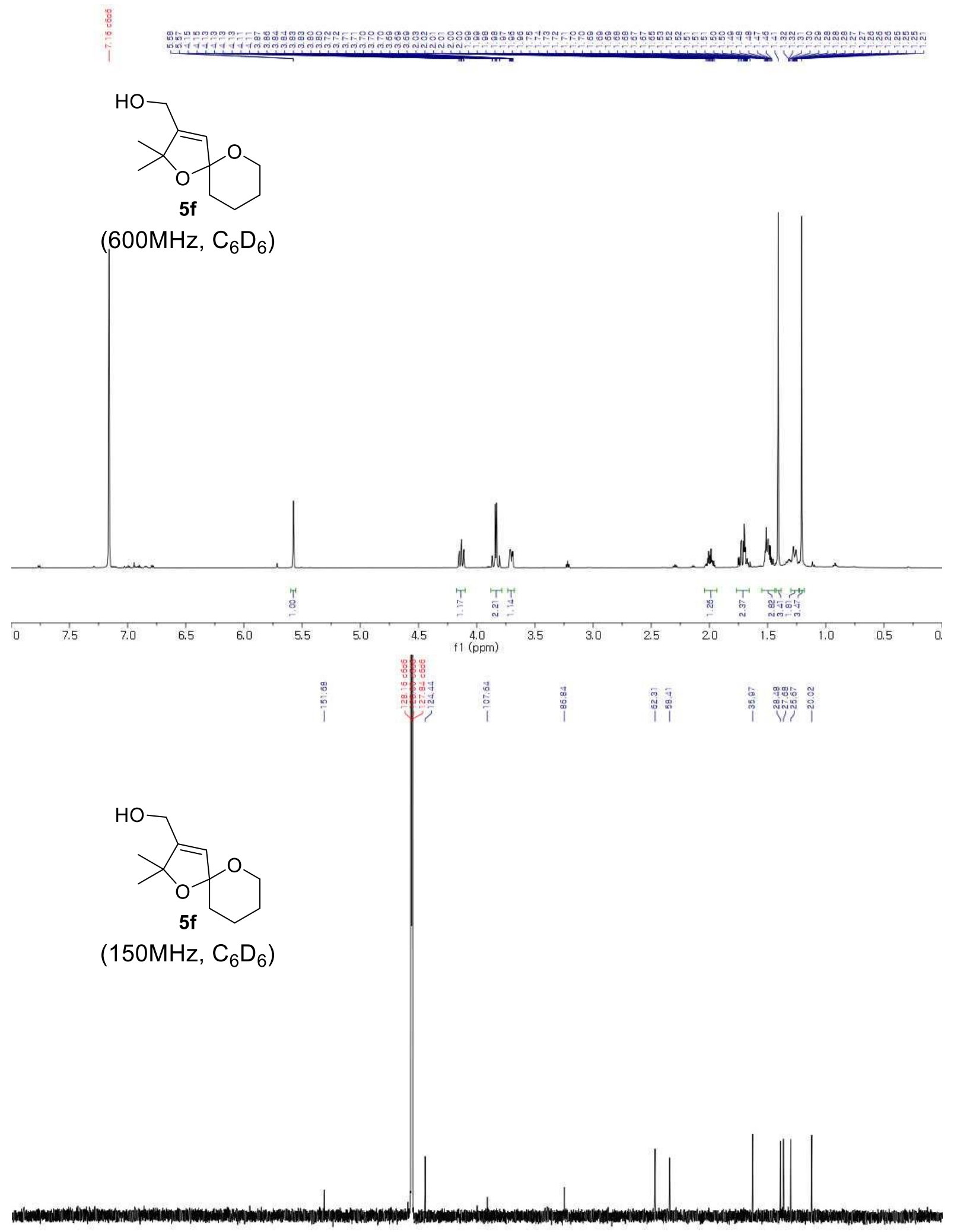

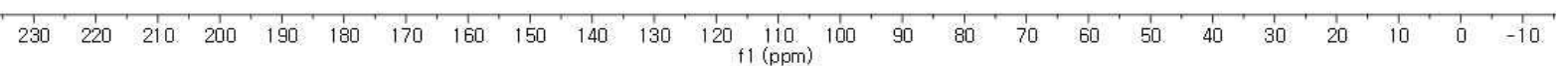




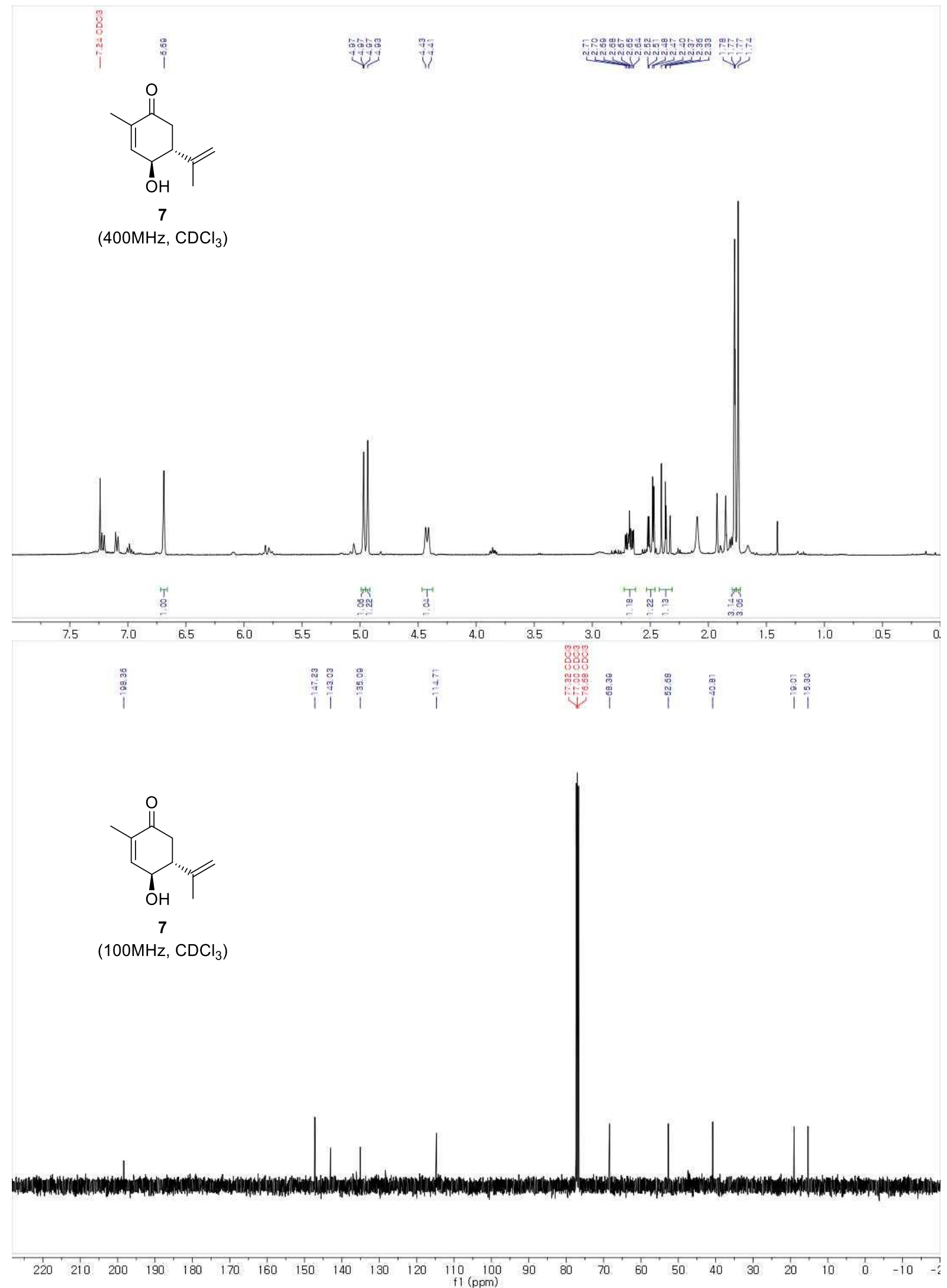




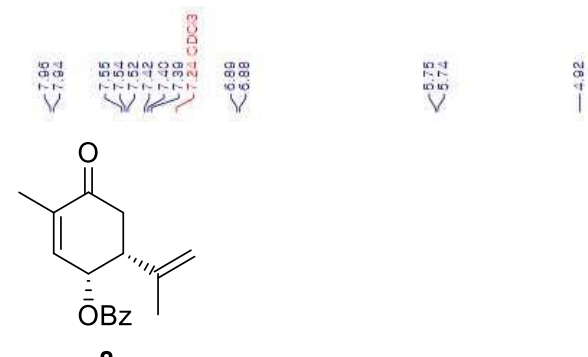

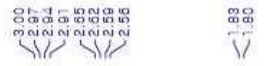

$\left(400 \mathrm{MHz}, \mathrm{CDCl}_{3}\right)$
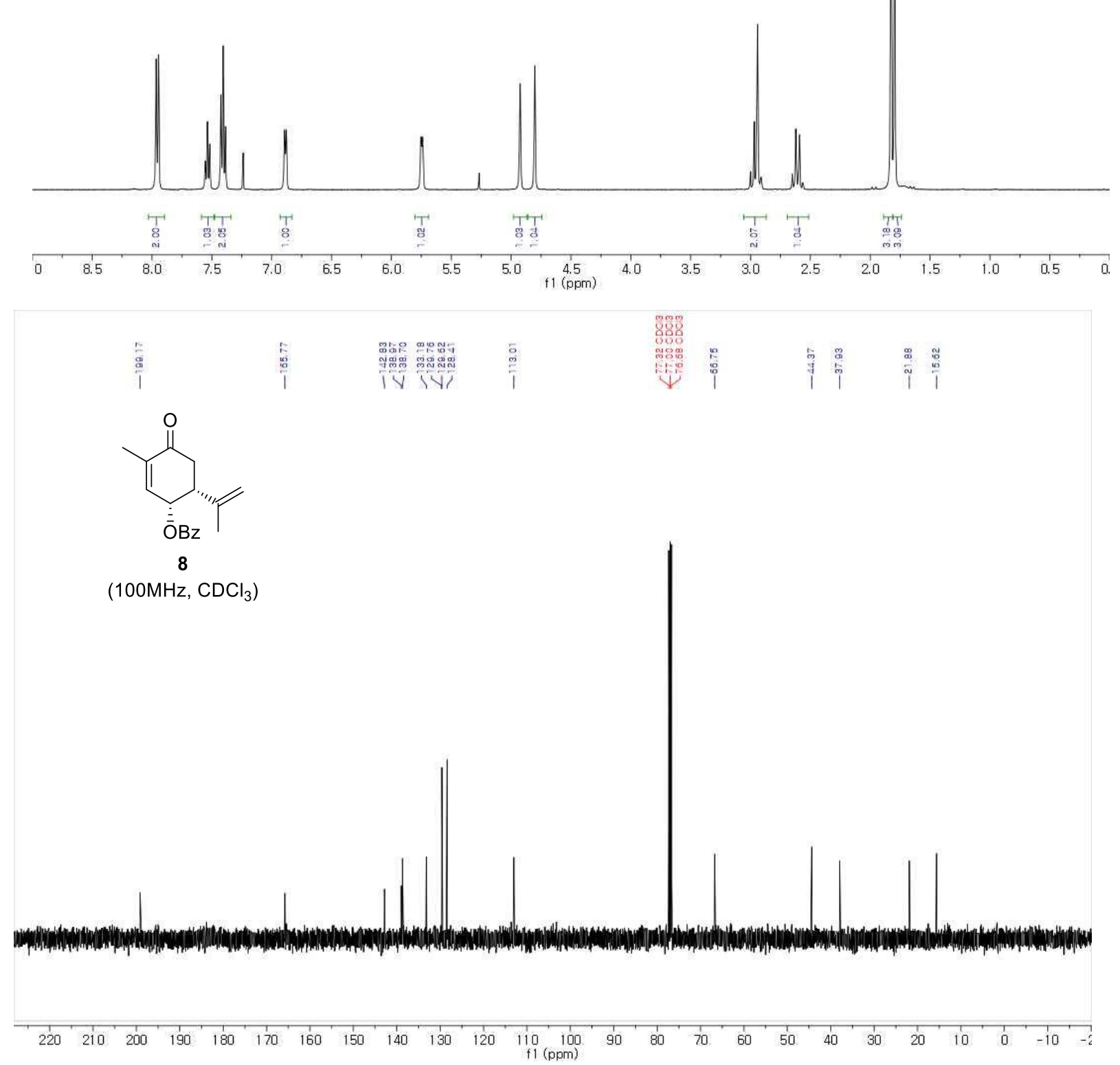

S-52 


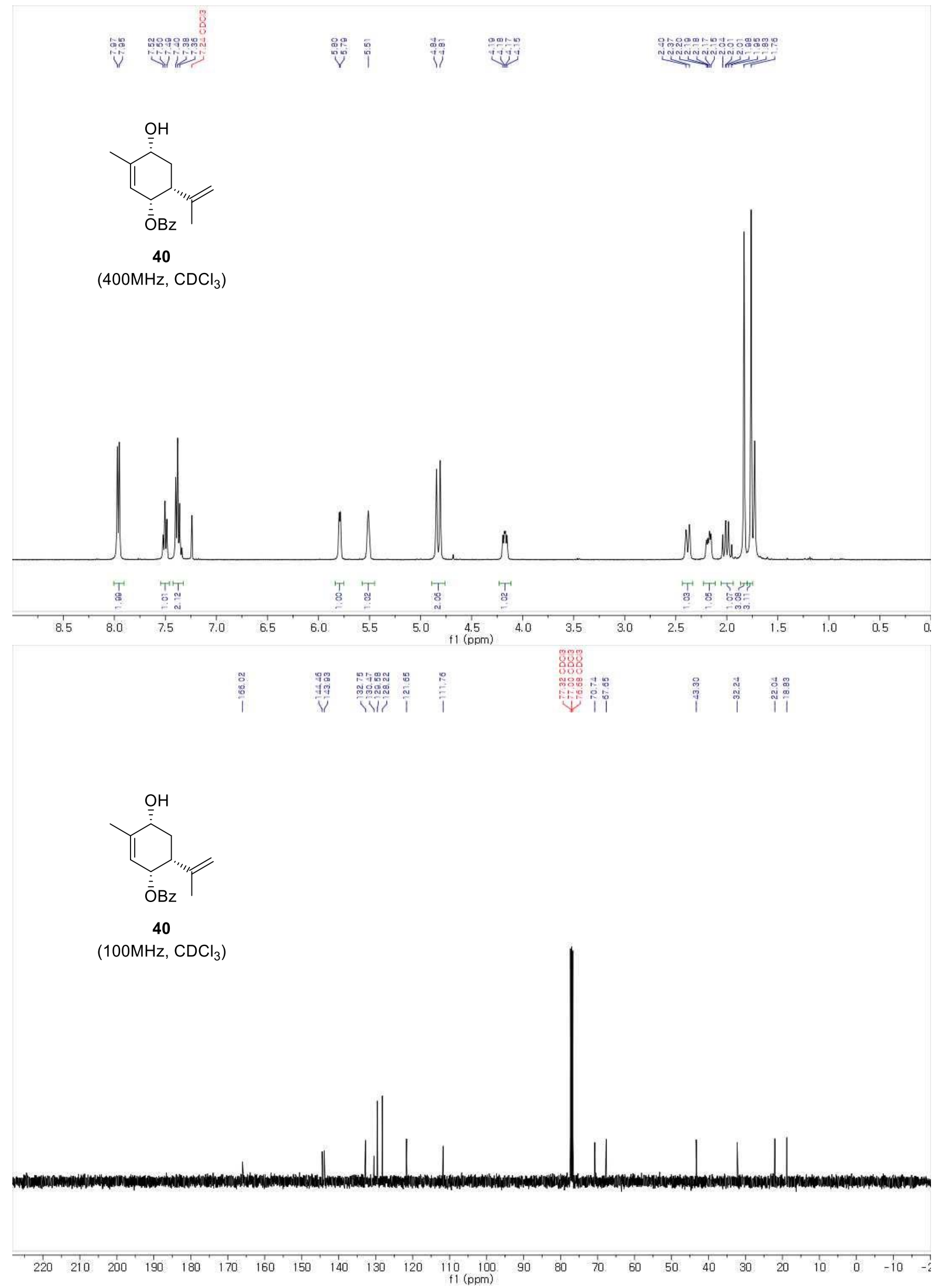



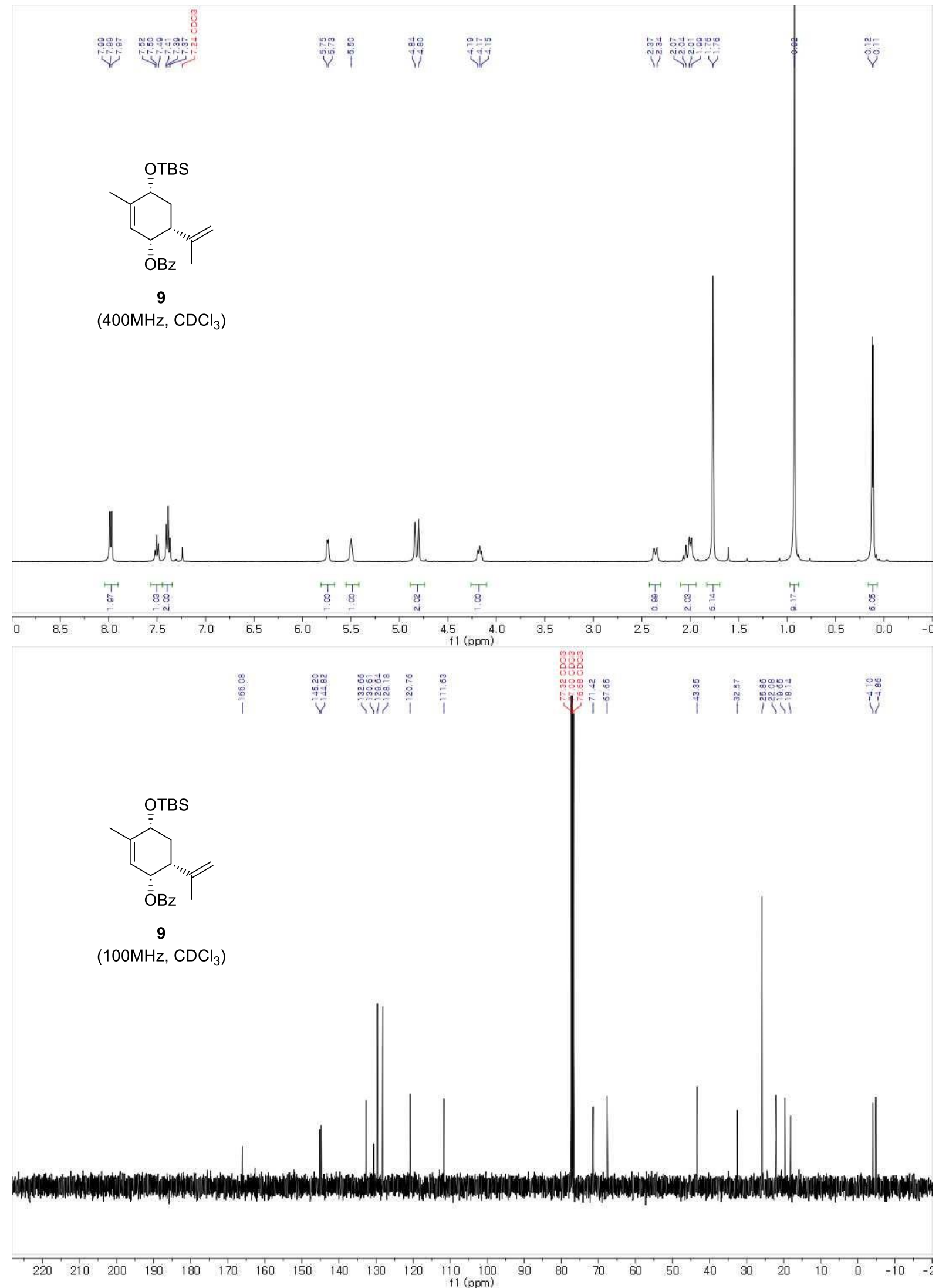


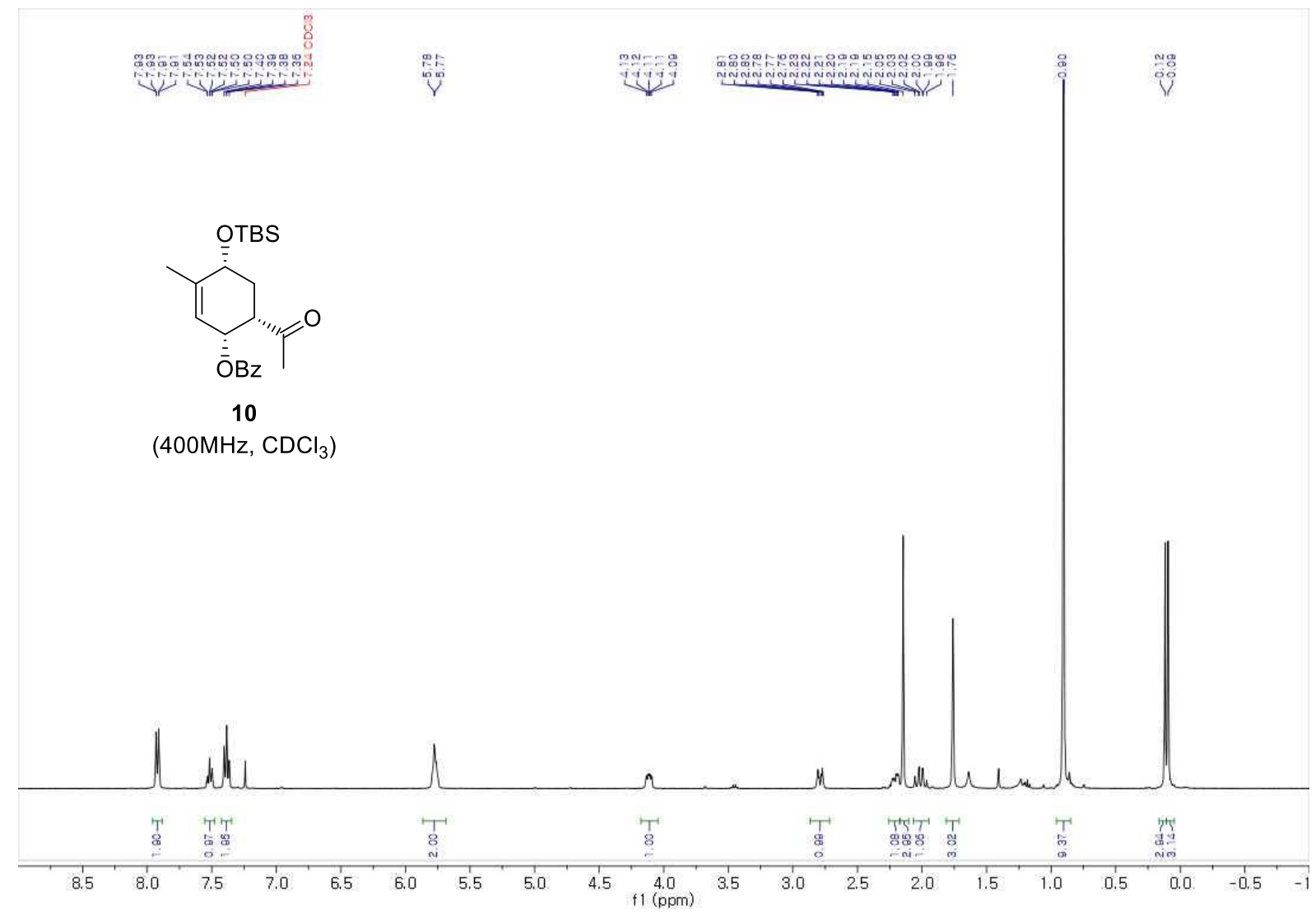

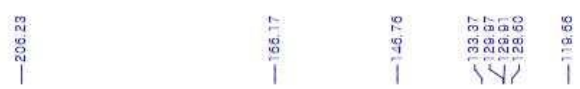

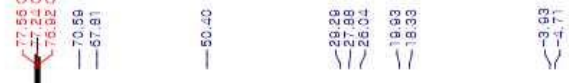<smiles>CC(=O)O[C@H]1C=C(C)[C@@H]([18OH])C[C@H]1C(C)=O</smiles>

10

(100MHz, $\mathrm{CDCl}_{3}$ )

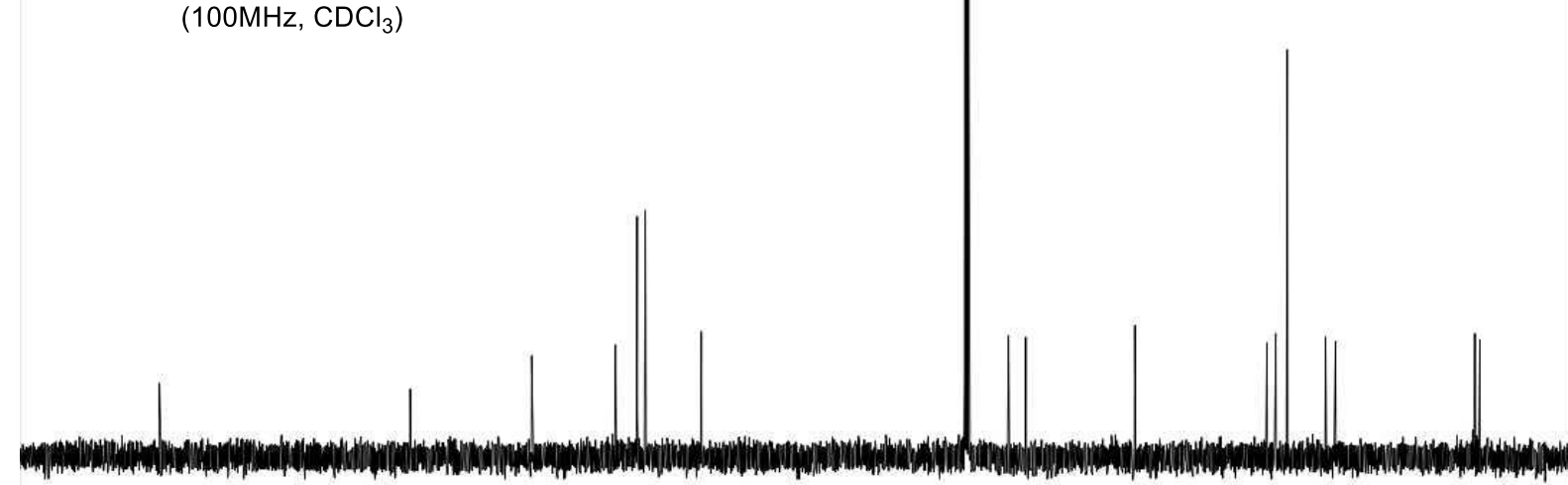

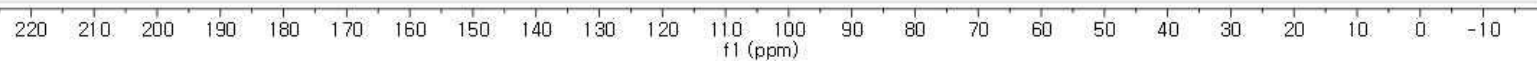



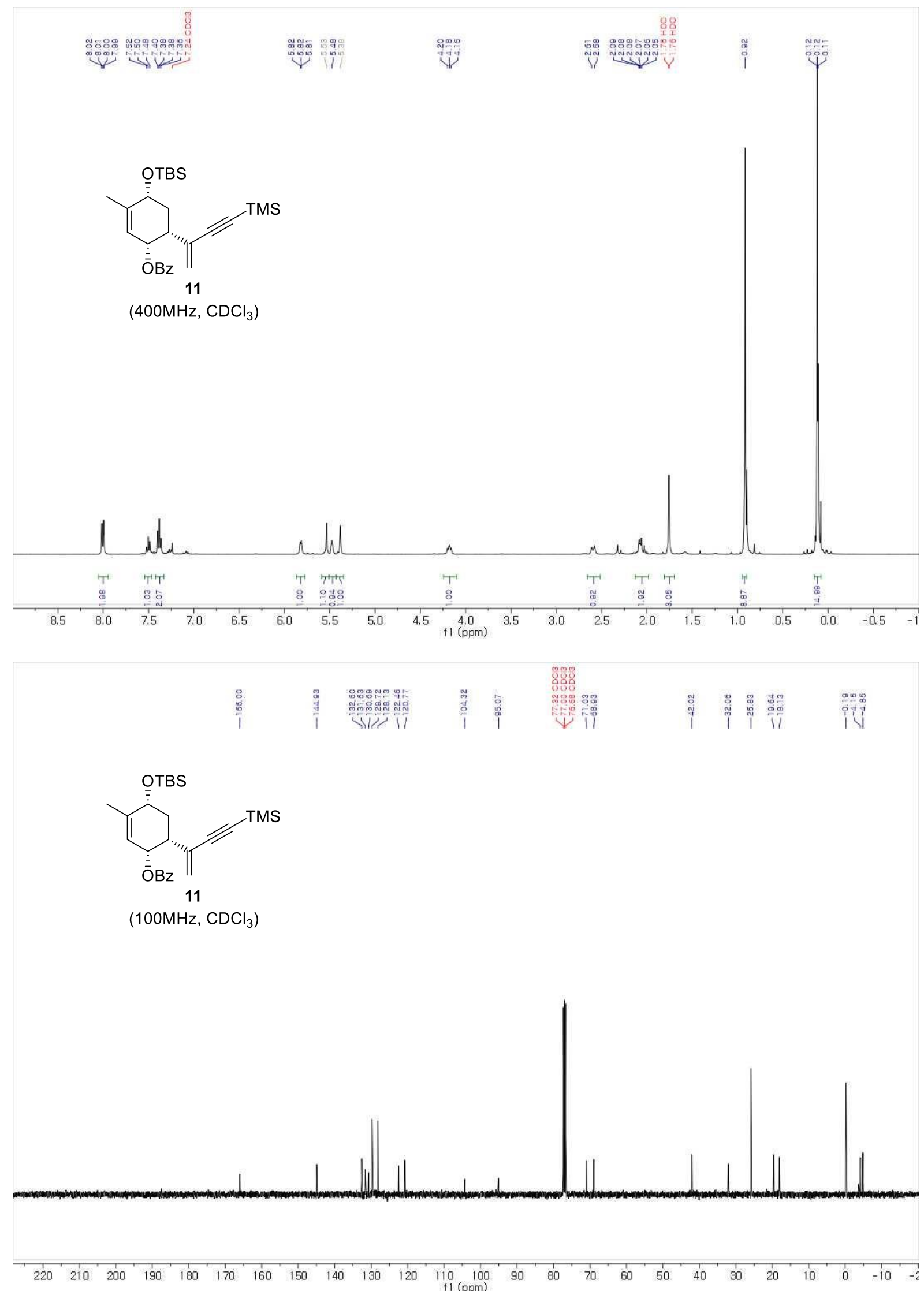

S-56 

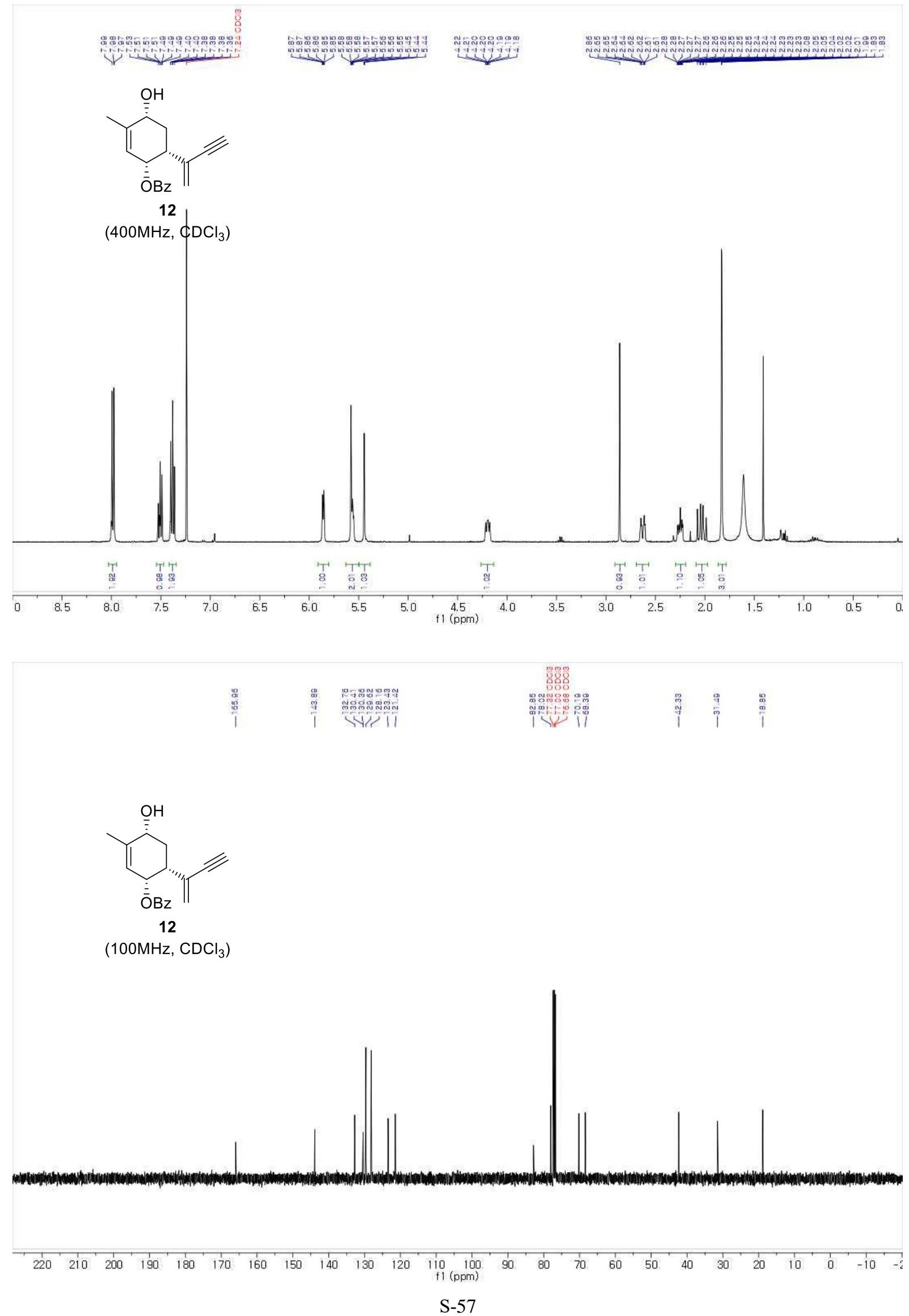

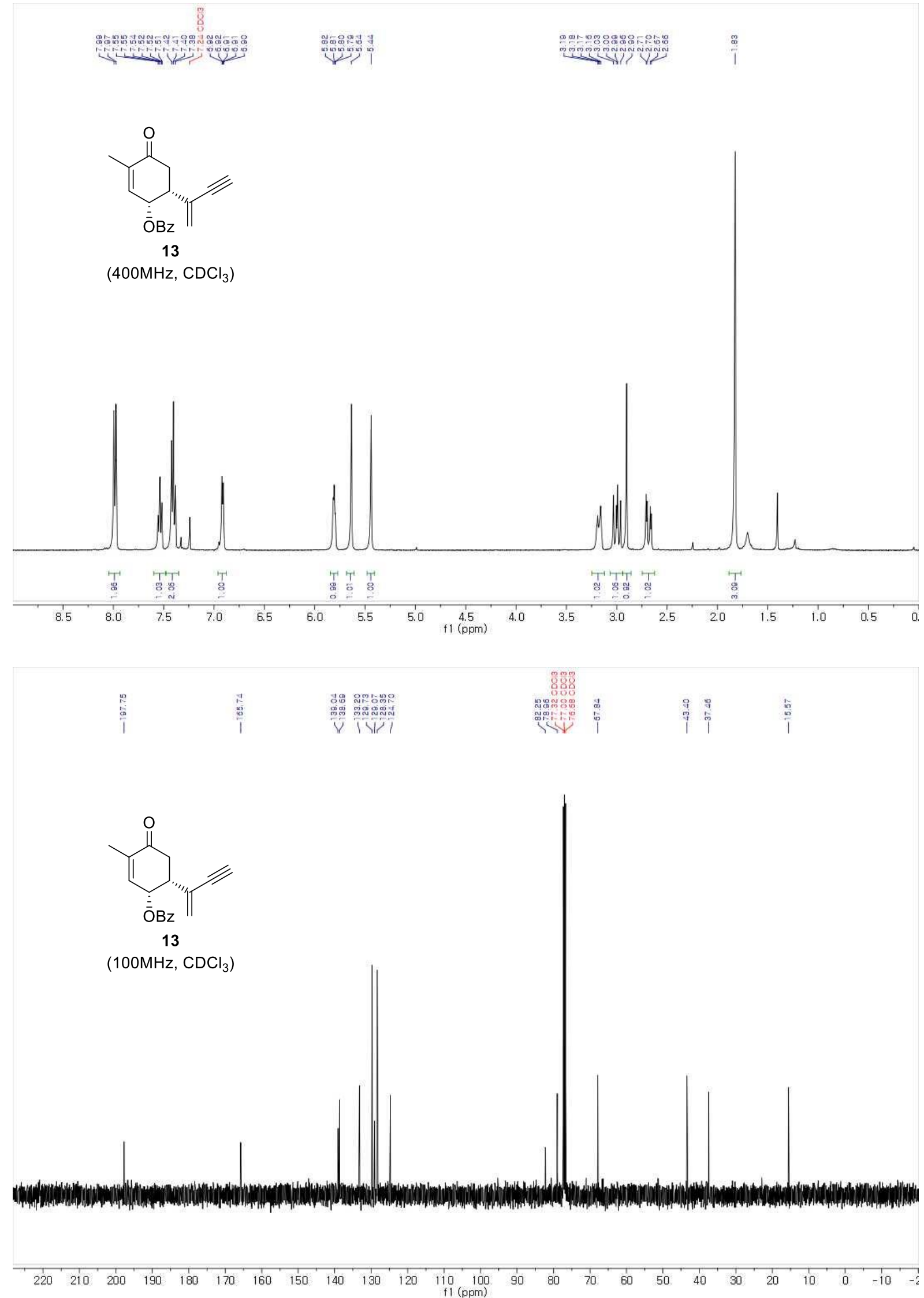

S-58 


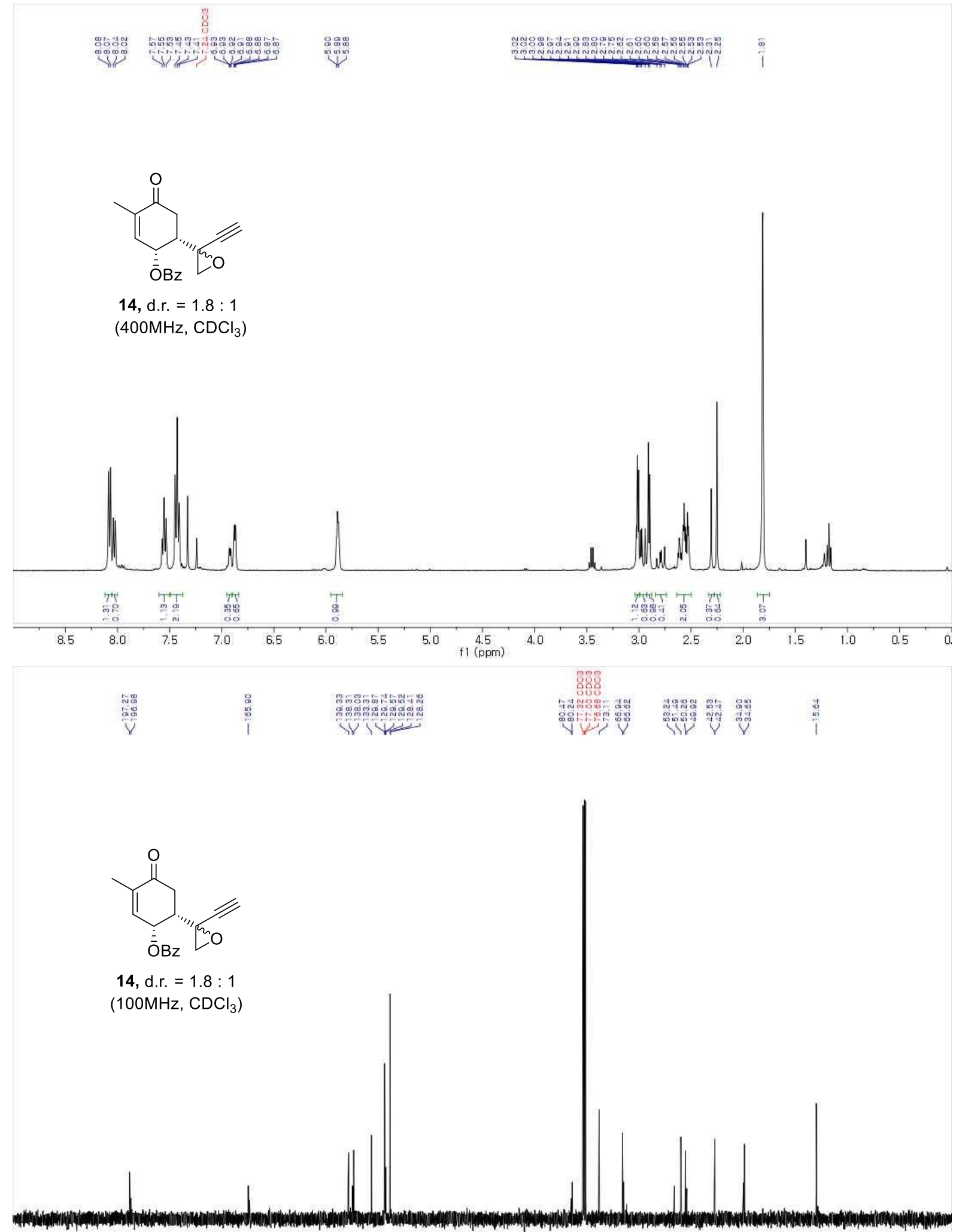

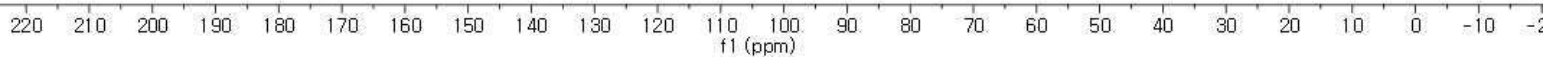



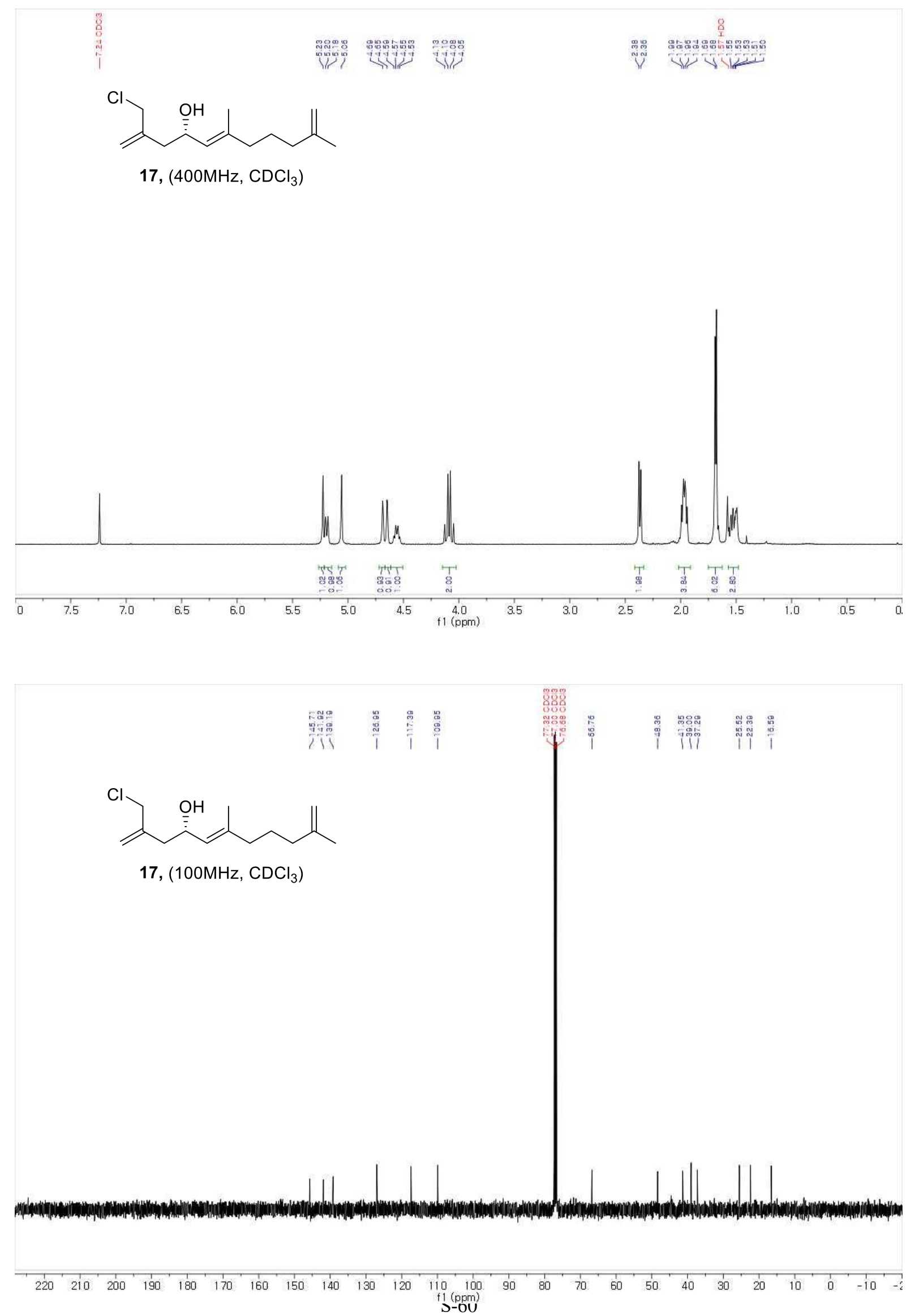

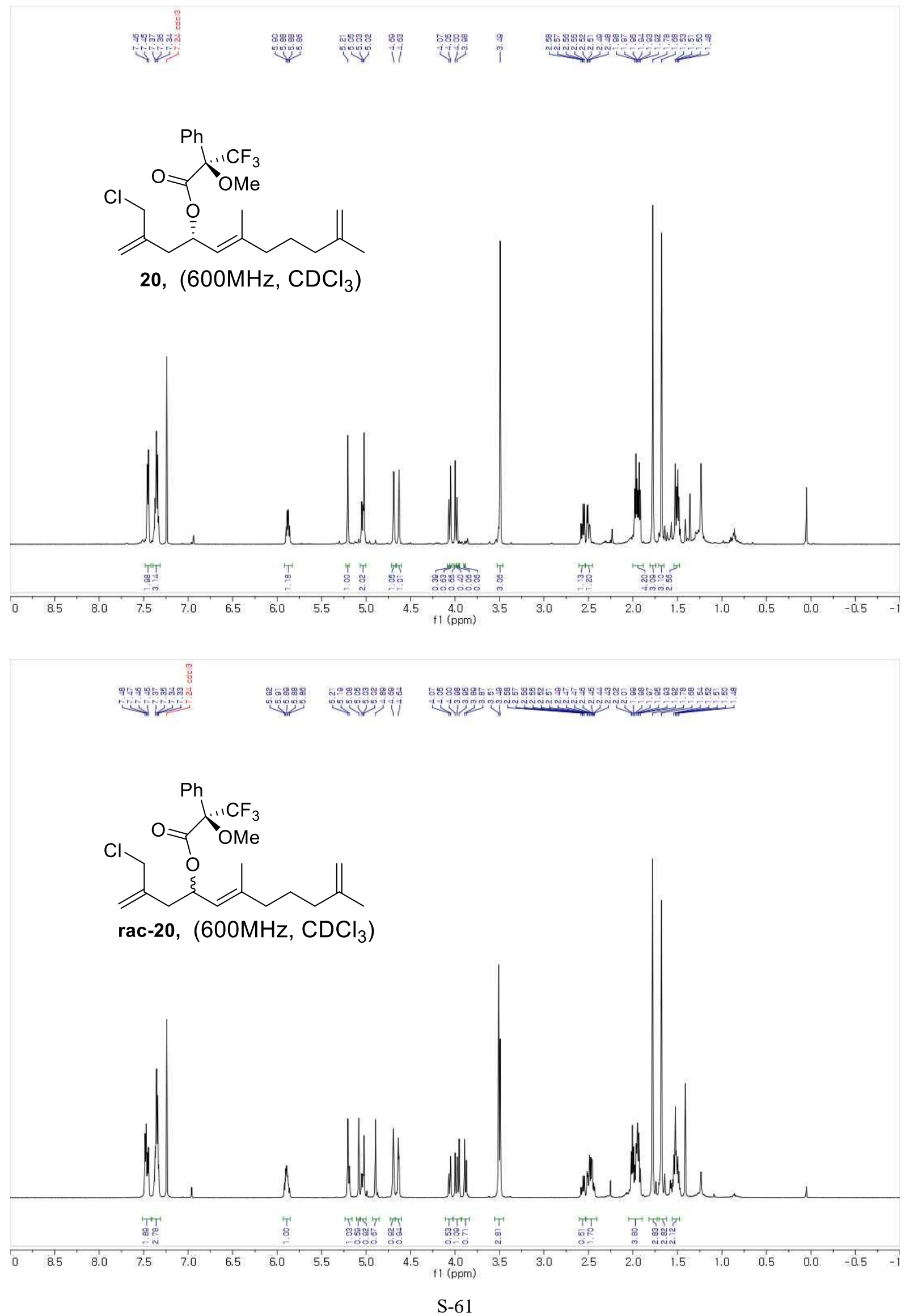


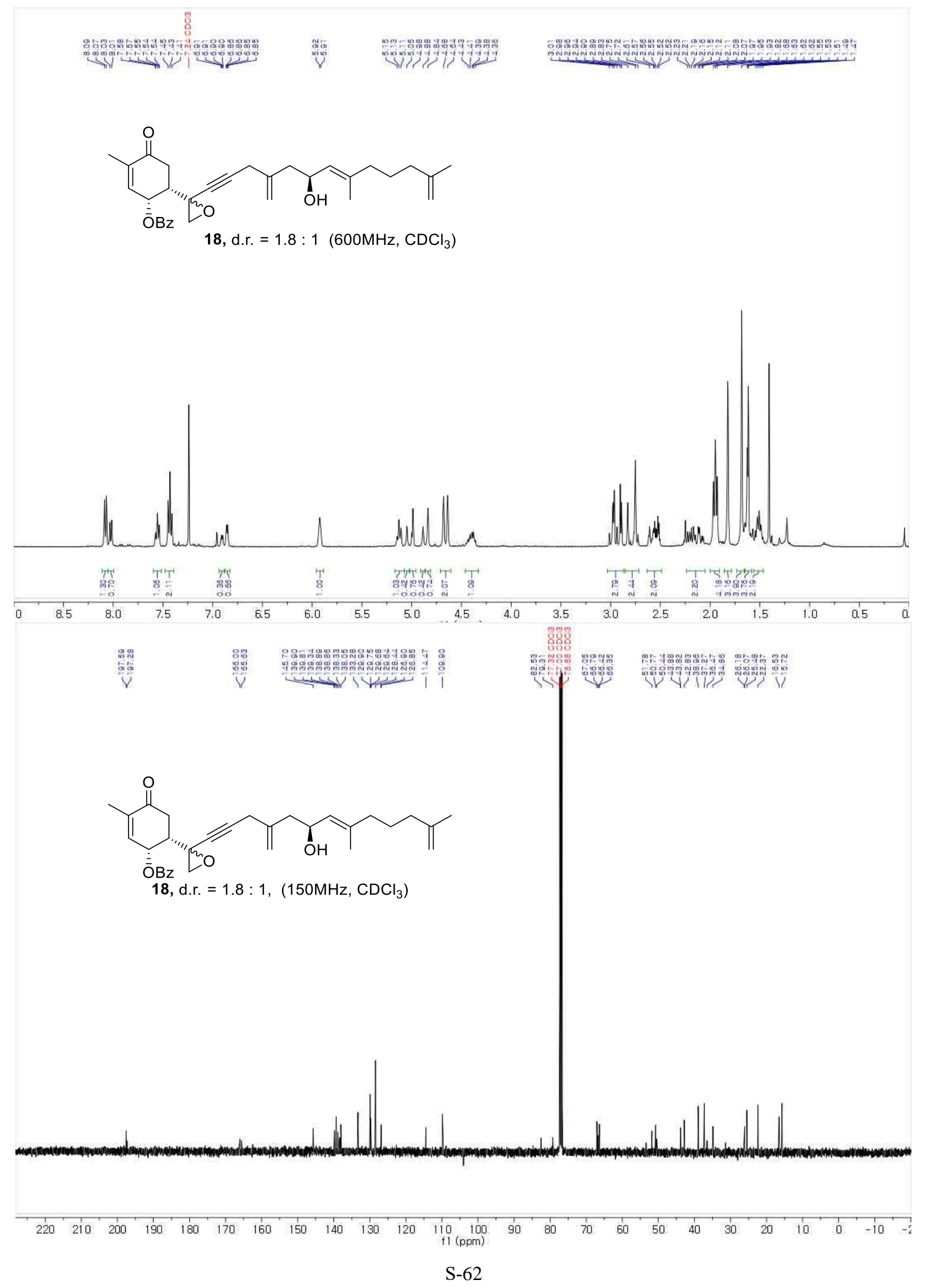



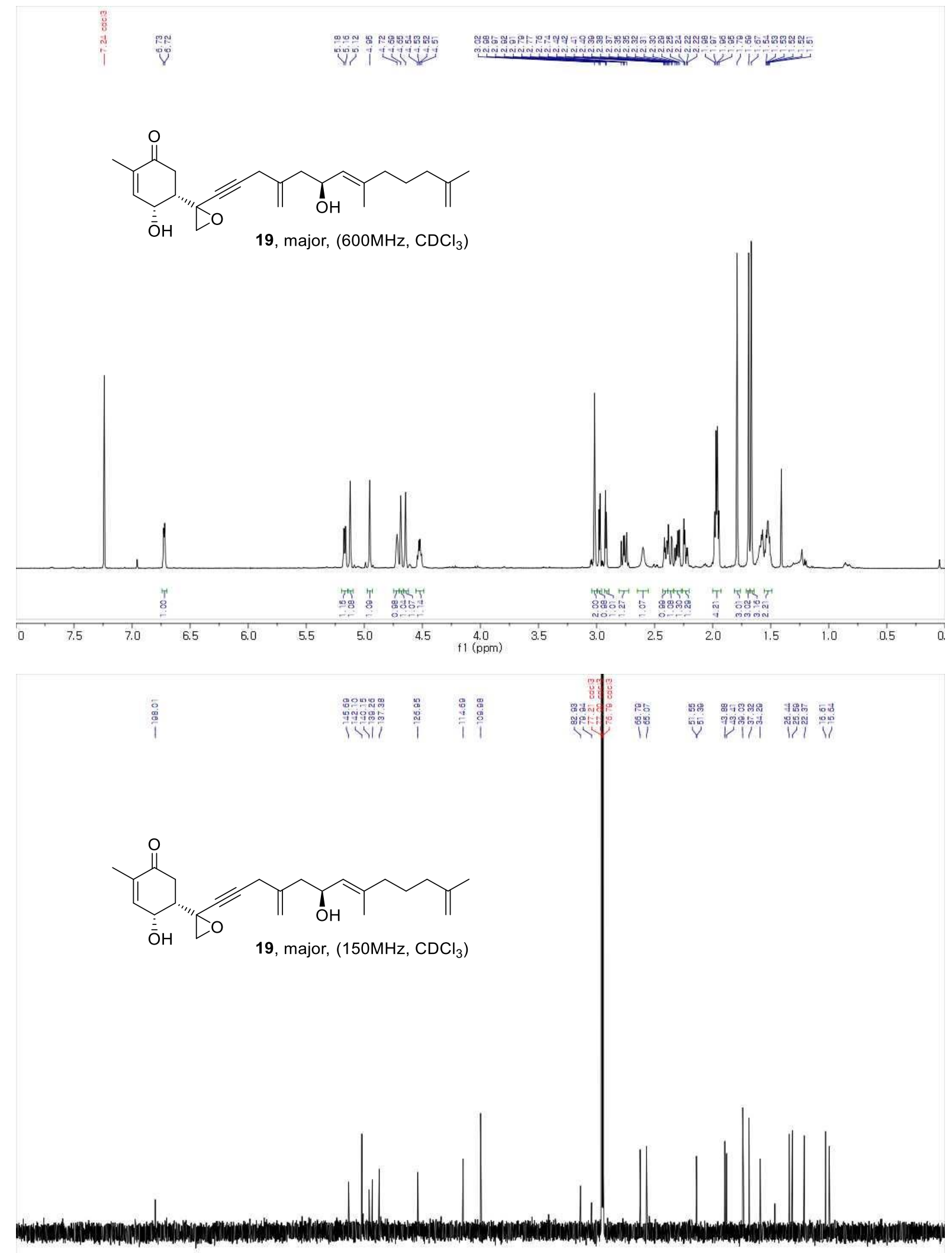

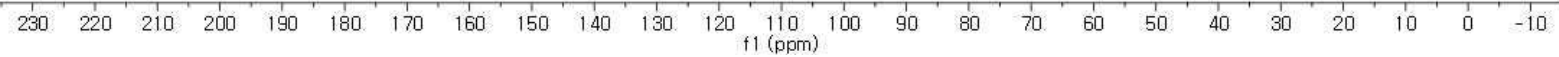



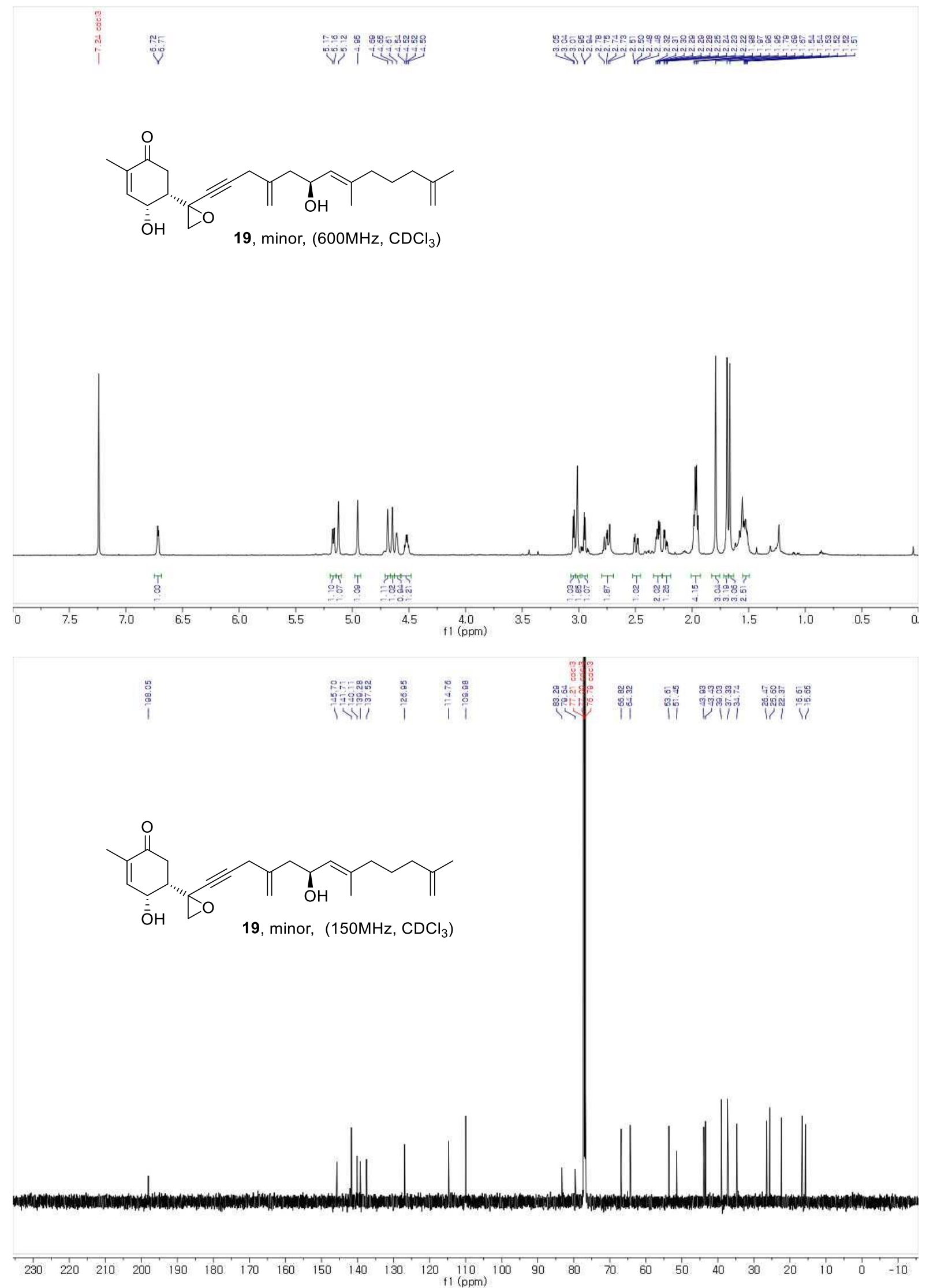


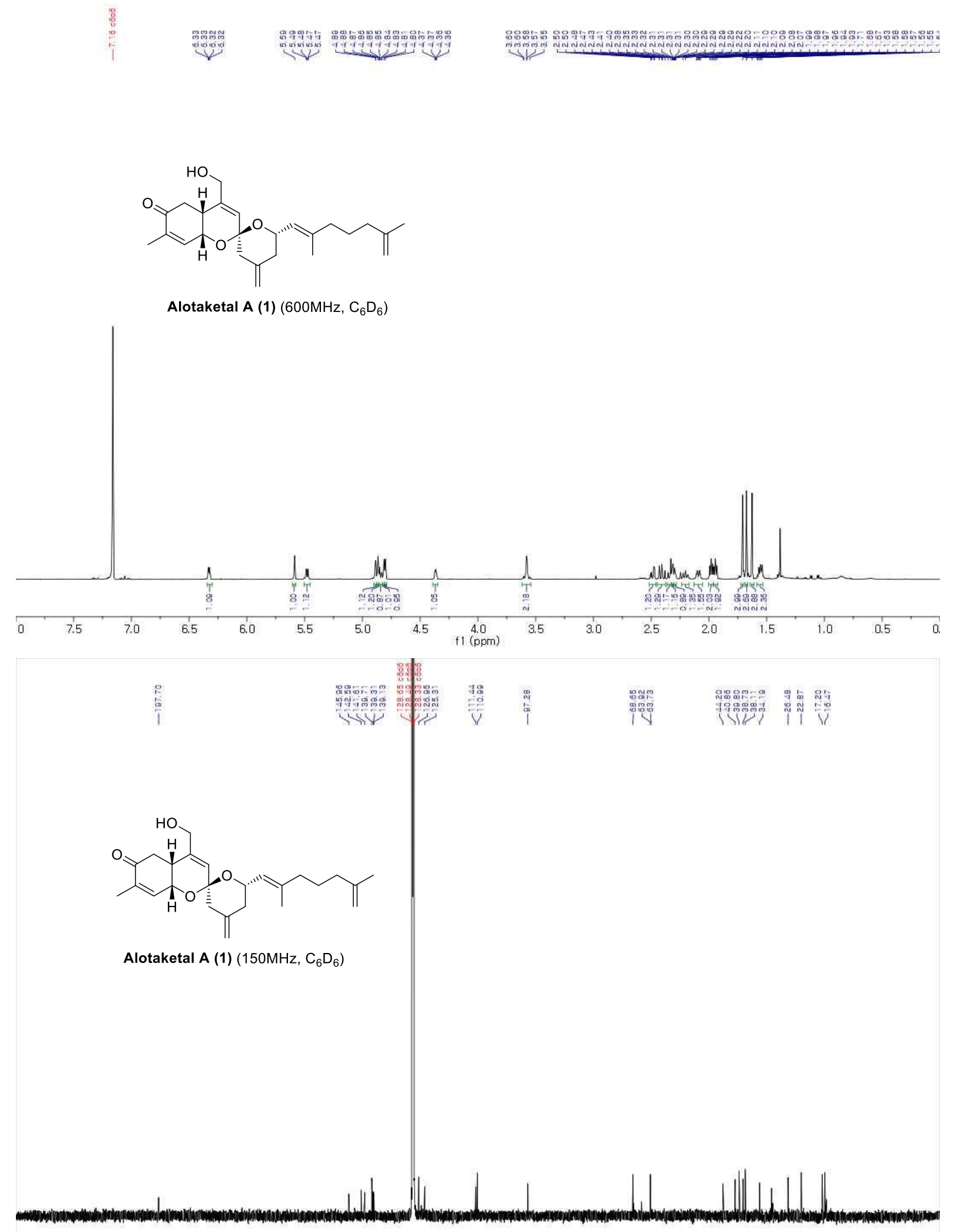

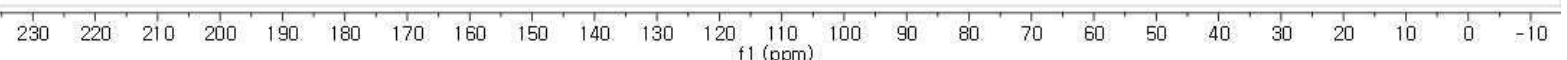




\section{References}

1. Xuan, M.; Paterson, I.; Dalby, S. M. Org. Lett. 2012, 14, 5492-5495

2. Hanawa, H.; Uraguchi, D.; Konishi. S.; Hashimoto, T.; Maruoka, K. Chem. Eur. J. 2003, 9, $4405-4413$ 